\title{
STATIC ECOLOGICAL SYSTEM ANALYSIS
}

\author{
HUSEYIN COSKUN*
}

A Holistic Analysis of Compartmental Systems

\begin{abstract}
In this article, a new mathematical method for static analysis of compartmental systems is developed in the context of ecology. The method is based on the novel system and subsystem partitioning methodologies through which compartmental systems are decomposed to the utmost level. That is, the distribution of environmental inputs and intercompartmental system flows, as well as the organization of the associated storages generated by these flows within the system is determined individually and separately. Moreover, the transient and the static direct, indirect, acyclic, cycling, and transfer (diact) flows and associated storages transmitted along a given flow path or from one compartment, directly or indirectly, to any other are analytically characterized, systematically classified, and mathematically formulated. A quantitative technique for the categorization of interspecific interactions and the determination of their strength within food webs is also developed based on the diact transactions. The proposed methodology allows for both input- and output-oriented analyses of static ecological networks. The input- and output-oriented analyses are introduced within the proposed mathematical framework and their duality is demonstrated. Major flow- and stock-related concepts and quantities of the current static network analyses are also integrated with the proposed measures and indices within this unifying framework. This comprehensive methodology enables a holistic view and analysis of ecological systems.
\end{abstract}

Key words. system decomposition theory, complex systems theory, ecological network analysis, compartmental systems, system and subsystem partitioning, subsystem scaling, transient flows and storages, diact flows and storages, food webs, interspecific interactions, input-output economics, socioeconomic systems, quantitative finance, epidemiology, infectious diseases, toxicology, pharmacokinetics, neural networks, chemical and biological systems, control theory, information theory, information diffusion, social networks, computer networks, malware propagation, graph theory, traffic flow

AMS subject classifications. 34A34, 35A24, 37N25, 37N40, 70G60, 91B74, 92B20, 92C42, 92D30, 92D40, 93C15

1. Introduction. Ecosystems are natural systems made up of living and nonliving components that work together. Ecosystem ecology is the study of the flow of energy or matter between these components and their environment based on conservation principles. Accurate and explicit formulation of flows and associated storages is of paramount importance for the quantification of ecosystem function. Ecosystem ecology also provides a framework for determining how local mechanisms interact with global environmental problems.

Environmental issues have taken center stage in human communities due to current scientific understandings of population and industrial growth, associated resource demands, and technological advances. Despite this increased attention to the environment, traditional ecology has an applied nature and is still in the empirical stage of development; a first principles-based formal theory has yet to emerge in its mainstream framework. This narrows the field's scope of applicability and compromises its ability to deal with complex organism-environment relationships. To that extent, ecology and environmental science are limited in their applied reach by a general inability to realistically model and analyze the complex systems of man and nature. Mathematical theories and modeling have significant potential to lead the way to a more formalistic and theoretical ecological science, devoted to the discovery of scientific laws. Based on this understanding and prediction, more exact, precise, and

\footnotetext{
*Department of Mathematics, University of Georgia, Athens, GA 30602 (hcoskun@uga.edu).
} 
incisive environmental applications can be expected to materialize.

Sound rationales have been offered in literature for ecological network analysis, but these are for specific cases, such as linear and static models. One such environmental system theory known as the environ theory, has been developed over recent decades for static compartmental models. Building on economic input-output analysis $[29,30]$ introduced into ecology by [21], the concepts of static flow and storage environs are formulated based on conservation principles [38, 33]. Along parallel research lines, ecological networks and complexity in living systems are also analyzed in the context of information theory and thermodynamics $[49,25,50,51]$ and hierarchy theory [1] yet only for static systems. Several software developments computerize these static methods $[52,8,16,28,43,6]$.

Although steady-state analyses are well-established, dynamic and nonlinear analytical methods have remained a long-standing, open problem. A mathematical theory and dynamic method recently proposed by $[12,10,11]$ potentially addresses the mismatch between current static and computational methods and applied ecological needs. In effect, this methodology brings a novel, formal, deterministic, complex system theory to the service of urgent ecological problems of the day. The main goal of the present manuscript is to introduce the static version of this dynamic methodology for the static ecosystem analysis.

The proposed mathematical method is based on the novel analytical and explicit, mutually exclusive and exhaustive system and subsystem partitioning methodologies. The system and subsystem partitioning methodologies determine the distribution of environmental inputs and intercompartmental flows, as well as the organization of the associated storages generated by these inputs and flows individually and separately within the system. In other words, these methodologies enable tracking the fate of environmental inputs and intercompartmental flows, as well as associated storages within the system. More specifically, the system partitioning methodology yields the subthroughflow and substorage matrices that respectively represent the flows and storages generated by individual environmental inputs at each compartment separately. Therefore, the system partitioning enables partitioning composite compartmental flows and storages into subcompartmental segments based on their constituent environmental sources. Through the subsystem partitioning methodology, then, the transient flows and associated storages transmitted along a given subflow path are formulated. Consequently, arbitrary composite intercompartmental flows and associated storages are decomposed into the constituent subflow and substorage segments along a given set of subflow paths within the subsystems.

Based on the concept of transient flow and storage, the static direct, indirect, acyclic, cycling, and transfer (diact) flows and associated storages transmitted from one compartment, directly or indirectly, to any other are also analytically characterized, systematically classified, and mathematically formulated for the quantification of intercompartmental flow and storage dynamics. The static subthroughflows and substorages, as well as the transient and diact flows and storages are systematically introduced in the present paper for the first time in literature. Only the cycling and transfer (total) flows were formulated in the literature [19, 20, 45], but the cycling and transfer storages generated by these flows are introduced in the present work. Equipped with these measures, the proposed methodology serves as a quantitative platform for testing empirical hypotheses, ecological inferences, and, potentially, theoretical developments. The method also constructs a base for the development of new system measures and indices as ecological indicators. Multiple such quantitative tools for the analysis of ecological network models are systematically introduced by [11, 13]. 
The proposed static methodology is a comprehensive approach through which the proposed measures and major flow- and stock-related concepts and quantities of ecological mathematics are combined and integrated effectively. Moreover, unique relationships among some current static measures are unveiled and corrections in some existing formulations are also suggested. The proposed methodology allows also for the analysis of static systems based on both their environmental inputs and outputs. The output-oriented analysis backtracks outputs instead of forward tracking inputs as the input-oriented analysis requires. Both input- and output-oriented ecosystem analyses are formulated through the system partitioning methodology and a subsystem scaling argument in the unifying setting of the proposed mathematical framework. Moreover, the duality of the input- and output-oriented analyses is demonstrated through novel similarity relationships. It should also be noted that while current static theories cannot analyze ecosystem models with time dependent environmental inputs, the proposed methodology can solve such linear systems analytically as formulated in Appendix A [10]. In summary, this is the first manuscript in literature that develops a holistic mathematical methodology for the analysis of ecological networks.

The indirect effects in ecosystems have long been a well-established empirical fact $[36,44,54,35,34,53]$. The theoretical ecological explorations of the concept began as early as the 1970s [27, 40,18, 32]. Although the indirect effects have been a topic of scholarly conversation for the past five decades, the indirect flows and storages have never been formulated before. The indirect effects are particularly important for the classification and characterization of interspecific interactions within food webs. The classification through direct relationships alone can turn out to be incorrect without holistically considering the entire network of interactions. Community ecology qualitatively describes interspecific interactions using network topology. On the other hand, for complex networks, such characterization becomes increasingly difficult, if possible at all $[55,35,34,26,7]$. A parametric characterization is proposed in the literature, however, it has some disadvantages due to the method formulation as detailed in Section 2.7 [48, 40, 17, 15, 47]. A novel quantitative technique for the characterization and classification of the neutral and antagonistic nature of direct and indirect interspecific interactions and, notably, for the determination of their strength is also developed as an immediate ecological application of the diact flows in the present manuscript.

The proposed methodology, therefore, can be used for quantitative analysis of complex food webs and food chains. The method enables tracking the fate of the same type of environmental nutrient or energy input into each species individually and separately within a food web. The evolution of an arbitrary amount of nutrient or energy in any species can also be tracked along a given food chain within the web. Moreover, the nutrient or energy transfers through interspecific interactions from one species directly or indirectly to another can be determined. Therefore, the spread of an arbitrary amount of nutrient or energy from one species to the entire network through trophic interactions along all or a particular food chain can be monitored. The input- and output-oriented analyses quantify the bottom-up and top-down activities within food webs, respectively. Additionally, while the input-oriented analysis determines the distribution of energy or nutrient flows and the organization of associated storages generated by environmental inputs within the web, the output-oriented analysis ascertains energy or nutrient flows and storages in each species destined to exit the web as dissipation or unusable waste.

The proposed method is applicable to any conservative compartmental system regardless of its nature, whether naturogenic or anthropogenic. The method, for 
example, can be applied to ecological system models designed for material flows in industrial ecology or dynamics of the terrestrial carbon cycle [3, 42]. It can also be used to quantitatively analyze complex food webs and food chains as outlined above and detailed in Sections 2.5 and 2.7 [4]. Although the motivating applications are ecological and environmental for this paper, the applicability of the proposed method extends to other realms, such as economics, pharmacology, epidemiology, chemical reaction kinetics, neural networks, biomedical systems, and information science -in fact, wherever compartmental models of conserved quantities can be constructed.

In order to present the efficiency of the proposed methodology, various models are analyzed in Section 3 and the Supplementary Materials. The first case study in Section 3 concerns energy transfer within the Cone Spring ecosystem, and the second examines nitrogen transfer within the Neuse River Estuary ecosystem. In either case, the distribution of both environmental inputs and intercompartmental flows of nitrogen and energy, as well as the organization of the associated storages generated by these inputs and flows within the ecosystems are analyzed through the proposed methodology. It is shown, for the Neuse River Estuary ecosystem, that the subcompartmental system measures can capture temporal dynamics, such as seasonality, better than the compartmental quantities. The fate of arbitrary nitrogen storages and the associated outflows emanating from these storages at each compartment along a given flow path are also determined for this ecosystem. For the Cone Spring ecosystem, not only along a given particular path, the spread of arbitrary environmental energy inputs throughout the system is demonstrated.

Analyzing direct transactions between compartments is relatively straightforward even in more complex systems. However, the analysis of indirect nitrogen or energy flow and storage transfers is made possible through the proposed methodology. The diact nitrogen and energy flows and associated storages transmitted from one compartment or species to any other, including itself, are presented for both ecosystem models. Moreover, it is shown that the proposed methodology can be used for the classification of ecosystems based on the dominance of one of the diact transaction types. The diact flows are also used to quantitatively classify the direct and indirect interspecific interactions between species, as well as to determine their strength. Through the analysis of the Neuse River Estuary ecosystem, it is shown that the strength of the interactions oscillate in time. It is also hypothesized that, possibly due to the slower biological activities during the winters, the transfer flows fall short of completing the nitrogen cycle and, therefore, the acyclic flows dominate the cycling flows within this ecosystem during the winters. Importantly, it is demonstrated that the ten percent energy transfer law from one to the next trophic level introduced by [31] is not an accurate estimate, at least for these ecosystems.

The Cone Spring ecosystem is also analyzed based on environmental outputs. The output-oriented system partitioning methodology enables partitioning the composite compartmental energy flows and storages into constituent subcompartmental segments destined to exit the system as environmental outputs separately via each species. The output-oriented subsystem partitioning then enables backtracking the fate of arbitrary energy storages and the associated inflows generating these storages at each species along a given food chain. Moreover, through the input-and outputoriented ecosystem analysis, energy flows and storages both generated and dissipated along all or a particular food chain are determined for this ecosystem. Some of the output-oriented diact flows and storages are also presented for this model. In addition to the Neuse River Estuary and Cone Spring ecosystems, the proposed methodology is also applied to a linear ecosystem model with time-dependent environmental in- 
put and a hypothetical static model in the Supplementary Materials (Section S5) to demonstrate various other uses and aspects of the proposed methodology.

The paper is organized as follows: the mathematical method is introduced in Section 2, case studies are provided in Section 3, and discussion and conclusions follow in Sections 4 and 5 . The dynamic methodology proposed by [10] is summarized below and in the Supplementary Materials.

2. Methods. A new mathematical theory and method has recently been developed for the dynamic analysis of nonlinear compartmental systems by [10, 12]. The terminology and notations used in this paper are adopted from [12]. The static version of the proposed dynamic methodology is formulated further below in this section.

The proposed theory is based on the novel system and subsystem partitioning methodologies. The system partitioning methodology yields the subthroughflow and substorage matrices for the distribution of environmental inputs and the organization of the associated storages generated by the inputs individually and separately within the system. For the quantification of the intercompartmental flow and storage dynamics, the subsystem partitioning methodology then formulates the transient and the static direct, indirect, acyclic, cycling, and transfer (diact) flows and associated storages transmitted along a given flow path or from one compartment directly or indirectly to any other. This mathematical method, therefore, as a whole, yields the decomposition of all system flows and storages to the utmost level.

A quantitative technique for the analysis of food chains and webs, as well as the characterization and classification of interspecific interactions and the determination of their strength is then developed based on the diact flows at the end of this section. We will start with a brief summary of the dynamic methodology introduced by [12, 10].

2.1. System partitioning methodology. The standard governing equations for compartmental dynamics are

$$
\dot{x}_{i}(t)=\left(z_{i}(t, x)+\sum_{j=1}^{n} f_{i j}(t, x)\right)-\left(y_{i}(t, x)+\sum_{j=1}^{n} f_{j i}(t, x)\right)
$$

with the initial conditions $x_{i}\left(t_{0}\right)=x_{i, 0}$, for $i=1, \ldots, n$. The concepts and notations employed in this formulation are as follows:

$$
\begin{array}{ll}
n & \text { number of compartments } \\
t & \text { time }[\mathrm{t}] \\
x_{i}(t) & \text { total material (mass) }[\mathrm{m}] \text { (or energy, currency) in compart- } \\
& \text { ment } i, i=1, \ldots, n \text {, at time } t \\
f_{i j}(t, x) & \text { nonnegative flow from compartment } j \text { to } i \text {, at time } t[\mathrm{~m} / \mathrm{t}] \\
y_{i}(t, x)=f_{0 i}(t, x) & \text { environmental }(j=0 \text { ) output from compartment } i \text { at time } \\
& t \\
z_{i}(t, x)=f_{i 0}(t, x) & \text { environmental input into compartment } i \text { at time } t
\end{array}
$$

For notational convenience, we define a direct flow matrix function $F$ of size $n \times n$ as

$$
F(t, x)=\left(f_{i j}(t, x)\right)
$$

and the inward and outward throughflow vector functions as

$$
\begin{aligned}
& \check{\tau}(t, x)=\left[\check{\tau}_{1}(t, x), \ldots, \check{\tau}_{n}(t, x)\right]^{T}=z(t, x)+F(t, x) \mathbf{1} \text { and } \\
& \hat{\tau}(t, x)=\left[\hat{\tau}_{1}(t, x), \ldots, \hat{\tau}_{n}(t, x)\right]^{T}=y(t, x)+F^{T}(t, x) \mathbf{1},
\end{aligned}
$$


respectively, where

$$
z(t, x)=\left[z_{1}(t, x), \ldots, z_{n}(t, x)\right]^{T} \quad \text { and } \quad y(t, x)=\left[y_{1}(t, x), \ldots, y_{n}(t, x)\right]^{T}
$$

are the input and output vector functions, $\mathbf{1}$ denotes the column vector of size $n$ whose entries are all one, and the superscript $T$ represents the matrix transpose.

The system partitioning methodology yields the governing equations for subcompartmental dynamics as follows (see Figs. 1 and 2):

$$
\dot{x}_{i_{k}}(t)=\left(z_{i_{k}}(t, \mathrm{x})+\sum_{j=1}^{n} f_{i_{k} j_{k}}(t, \mathrm{x})\right)-\left(y_{i_{k}}(t, \mathrm{x})+\sum_{j=1}^{n} f_{j_{k} i_{k}}(t, \mathrm{x})\right)
$$

for $i=1, \ldots, n, k=0, \ldots, n$, with the initial conditions

$$
x_{i_{k}}\left(t_{0}\right)=\left\{\begin{array}{rr}
x_{i, 0}, & k=0 \\
0, & k \neq 0
\end{array}\right.
$$

where

$$
\mathrm{x}(t)=\left[x_{1_{0}}(t), \ldots, x_{n_{0}}(t), x_{1_{1}}(t), \ldots, x_{n_{1}}(t), \ldots, x_{1_{n}}(t), \ldots, x_{n_{n}}(t)\right]^{T} .
$$

The relationship between the compartmental and subcompartmental flows and storages can be stated as

$$
x_{i}(t)=\sum_{k=0}^{n} x_{i_{k}}(t) \quad \text { and } \quad f_{i j}(t, x)=\sum_{k=0}^{n} f_{i_{k} j_{k}}(t, \mathrm{x})
$$

where

$$
f_{i_{k} j_{k}}(t, \mathrm{x})=x_{j_{k}}(t) \frac{f_{i j}(t, x)}{x_{j}(t)}=d_{j_{k}}(\mathrm{x}) f_{i j}(t, x)
$$

and the decomposition factor is defined as $d_{j_{k}}(\mathrm{x})=x_{j_{k}}(t) / x_{j}(t)$.

The concepts and notations used in the system partitioning methodology are summarized below:

$$
\begin{array}{ll}
x_{i_{k}}(t) & \text { storage generated by environmental input } z_{k}(t, x) \text { during } \\
& {\left[t_{0}, t\right] \text { and stored in subcompartment } k \text { of compartment } i,} \\
& \text { that is, in subcompartment } i_{k}, k=0, \ldots, n \text {, at time } t \\
& \text { nonnegative flow from subcompartment } j_{k} \text { to } i_{k} \text { at time } t \\
f_{i_{k} j_{k}}(t, \mathrm{x}) & \text { environmental }(j=0) \text { output from subcompartment } i_{k} \text { at } \\
y_{i_{k}}(t, \mathrm{x})=f_{0 i_{k}}(t, \mathrm{x}) & \text { time } t \\
z_{i_{k}}(t, \mathrm{x})=\delta_{i k} z_{i}(t, x) & \text { environmental input into subcompartment } i_{k} \text { at time } t, \\
& \text { where } \delta_{i k} \text { is the discrete delta function }
\end{array}
$$

Thus, for each $k$, we explicitly generate a subsystem running within the system through the dynamic system partitioning methodology. This $k^{\text {th }}$ subsystem is composed of all $k^{t h}$ subcompartments and their corresponding substorages and subflows. These mutually exclusive and exhaustive subsystems have the same structure and dynamics as the original system, except for their environmental inputs and initial conditions (see Figs. 1 and 2). By mutual exclusiveness, we mean that transactions 

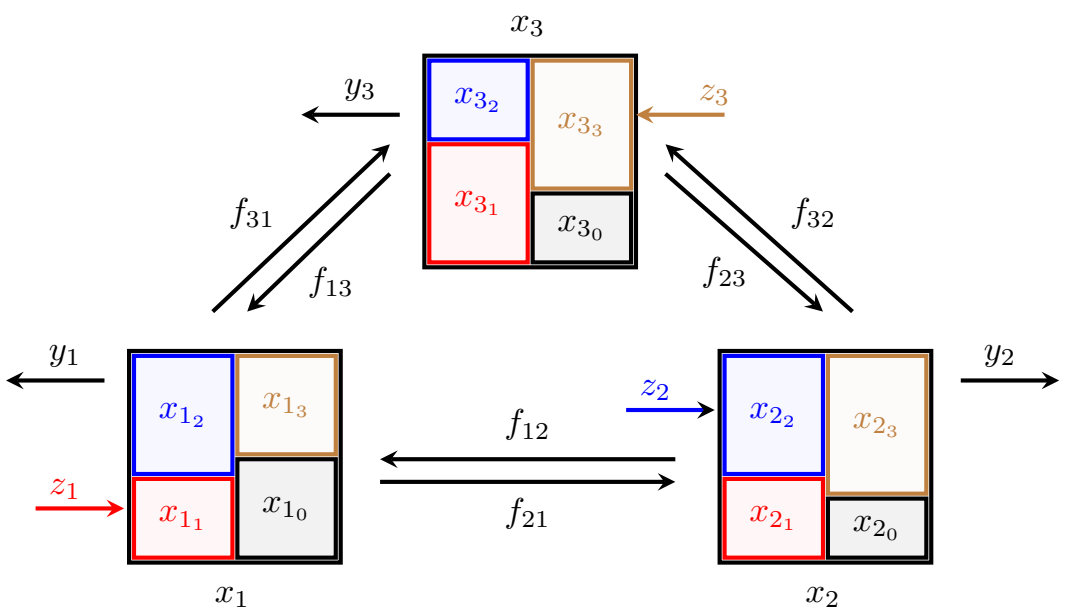

FIG. 1. Schematic representation of the input-oriented dynamic subcompartmentalization in a three-compartment model system. Each subsystem is colored differently; the second subsystem $(k=2)$ is blue, for example. Only the subcompartments in the same subsystem $\left(x_{1_{2}}(t), x_{2_{2}}(t)\right.$, and $x_{3_{2}}(t)$ in the second subsystem, for example) interact with each other. Subsystem $k$ receives environmental input only at subcompartment $k_{k}$. The initial subsystem receives no environmental input. The dynamic flow partitioning is not represented in this figure. Compare this figure with Fig. 2, in which the subcompartmentalization and the corresponding flow partitioning are illustrated for $x_{1}(t)$ only.

are possible only within corresponding subcompartments of the same subsystem. By exhaustiveness, we mean that all the generated subsystems sum to the entire system so partitioned. Except the initial subsystem, which is driven by all initial stocks, each subsystem is driven by a single environmental input. Therefore, the number of subcompartments in each compartment is equal to the number of inputs (or compartments), plus one for the initial stocks. If an input or all initial conditions are zero, the corresponding subsystem is null. Consequently, in a system with $n$ compartments, each compartment has $n+1$ subcompartments, and, therefore, the system has $n+1$ subsystems, indexed by $k=0, \ldots, n$. The initial subsystem $(k=0)$ represents the evolution of the initial stocks, receives no environmental input, and has the same initial conditions as the original system. The initial conditions for all the other subcompartments are zero.

If the original system, Eq. 2.1, is linear, the decomposed system, Eq. 2.3, can be expressed in the following linear matrix form:

$$
\begin{array}{ll}
\dot{X}(t)=\check{T}(t)-\hat{T}(t), & X\left(t_{0}\right)=\mathbf{0}, \\
\dot{x}_{0}(t)=\check{\tau}_{0}(t)-\hat{\tau}_{0}(t), & x_{0}\left(t_{0}\right)=x_{0},
\end{array}
$$

where

$$
X_{i k}(t)=x_{i_{k}}(t), \quad \check{T}_{i k}(t)=\check{\tau}_{i_{k}}(t), \quad \text { and } \quad \hat{T}_{i k}(t)=\hat{\tau}_{i_{k}}(t)
$$

for $i, k=1, \ldots, n$; the inward and outward subthroughflows are

$$
\check{\tau}_{i_{k}}(t)=z_{i_{k}}(t)+\sum_{j=1}^{n} f_{i_{k} j_{k}}(t) \quad \text { and } \quad \hat{\tau}_{i_{k}}(t)=y_{i_{k}}(t)+\sum_{j=1}^{n} f_{j_{k} i_{k}}(t)
$$




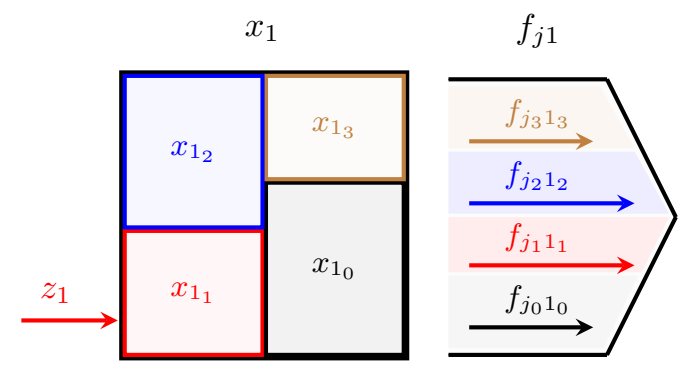

FIG. 2. Schematic representation of the dynamic flow partitioning in a three-compartment model system. The figure illustrates subcompartmentalization of compartment $i=1$ and the corresponding dynamic flow partitioning from this compartment to others, $j$.

for $k=0,1, \ldots, n$; and the storage, inward, and outward throughflow vector functions for the initial subsystem are $x_{0}(t)=\left[x_{1_{0}}(t), \ldots, x_{n_{0}}(t)\right]^{T}, \check{\tau}_{0}(t)=\left[\check{\tau}_{1_{0}}(t), \ldots, \check{\tau}_{n_{0}}(t)\right]^{T}$, and $\hat{\tau}_{0}(t)=\left[\hat{\tau}_{1_{0}}(t), \ldots, \hat{\tau}_{n_{0}}(t)\right]^{T}$, respectively. We use the constant notation $x_{0}$ for the constant initial conditions and the function notation $x_{0}(t)$ for the evolution of these initial conditions with $x_{0}\left(t_{0}\right)=x_{0}$. The flows and storages for the $k^{\text {th }}$ subsystem in matrix form are formulated in Appendix B for systems at steady state.

The governing system, Eq. 2.6, can alternatively be expressed as follows:

$$
\begin{aligned}
\dot{X}(t) & =\mathcal{Z}(t)+A(t) X(t), & & X\left(t_{0}\right)=\mathbf{0} \\
\dot{x}_{0}(t) & =A(t) x_{0}(t), & & x_{0}\left(t_{0}\right)=x_{0}
\end{aligned}
$$

where

$$
A(t)=\left(F(t)-\mathcal{Y}(t)-\operatorname{diag}\left(F^{T}(t) \mathbf{1}\right)\right) \mathcal{X}^{-1}(t),
$$

and the diagonal storage, input, and output matrices are

$$
\mathcal{X}(t)=\operatorname{diag}(x(t)), \quad \mathcal{Y}(t)=\operatorname{diag}(y(t)), \quad \text { and } \quad \mathcal{Z}(t)=\operatorname{diag}(z(t)) .
$$

The notation $\operatorname{diag}(x(t))$ represents the diagonal matrix whose diagonal elements are the elements of vector $x(t)$, and $\operatorname{diag}(X(t))$ represents the diagonal matrix whose diagonal elements are the same as the diagonal elements of matrix $X(t)$.

The subthroughflow matrices can then be formulated as

$$
\begin{aligned}
\check{T}(t) & =\mathcal{Z}(t)+F(t) \mathcal{X}^{-1}(t) X(t), \\
\hat{T}(t) & =\left(\mathcal{Y}(t)+\operatorname{diag}\left(F^{T}(t) \mathbf{1}\right)\right) \mathcal{X}^{-1}(t) X(t) \\
& =\mathcal{T}(t) \mathcal{X}^{-1}(t) X(t),
\end{aligned}
$$

using Eqs. 2.5 and 2.8, where $\mathcal{T}(t)=\operatorname{diag}(\hat{\tau}(t))=\mathcal{Y}(t)+\operatorname{diag}\left(F^{T}(t) \mathbf{1}\right)$. Note that,

$$
\begin{aligned}
& x(t)=x_{0}(t)+X(t) \mathbf{1}, \\
& \check{\tau}(t)=\check{\tau}_{0}(t)+\check{T}(t) \mathbf{1}, \quad \text { and } \quad \hat{\tau}(t)=\hat{\tau}_{0}(t)+\hat{T}(t) \mathbf{1} .
\end{aligned}
$$

It is worth emphasizing that current static methodologies cannot analyze systems with time-dependent inputs. The proposed dynamic methodology, however, can solve linear systems analytically. Analytic solutions for linear systems, Eq. 2.9, with timedependent environmental inputs are formulated in Appendix A, and a linear dynamic model introduced by [24] is worked out as an illustrative example in the Supplementary Materials (Section S5.1). 
2.2. Steady-state solution. At steady state, the time derivatives are zero and all system flows and storages are constant. That is,

$$
\dot{X}(t)=\mathbf{0} \quad \text { and } \quad \dot{x}_{0}(t)=\mathbf{0} .
$$

We will use the same notation, $\mathbf{0}$, for both zero matrix and zero vector for notational simplicity. If the partitioned system is at steady state, the original system is also at steady state. The constant static quantities will be denoted by the same symbols without the time argument, that is $X(t)=X$, for example.

For linear systems, $A$ is a strictly diagonally dominant constant matrix and, therefore, is invertible. It can be expressed as follows:

$$
A=(F-\mathcal{T}) \mathcal{X}^{-1}=F \mathcal{X}^{-1}-\mathcal{T} \mathcal{X}^{-1}=Q^{x}-\mathcal{R}^{-1}
$$

where $Q^{x}=F \mathcal{X}^{-1}, \mathcal{R}=\mathcal{X} \mathcal{T}^{-1}$, and $\mathcal{T}=\operatorname{diag}(\hat{\tau})=\mathcal{Y}+\operatorname{diag}\left(F^{T} \mathbf{1}\right)$. Note that the first term in the definition of $A, Q^{x}$, represents the intercompartmental flow intensity, and the second term, $\mathcal{R}^{-1}$, represents the outward throughflow intensity. Therefore, the $n \times n$ matrix $A$ will be called the flow intensity matrix per unit storage. It is sometimes called the compartmental matrix. The matrices $Q^{x}$ and $\mathcal{R}$ will be discussed in detail in Section 2.4.

We then have the following solutions to Eq. 2.9 for the substorage matrix, $X$, and initial substorage vector, $x_{0}$, at steady state:

$$
X=-A^{-1} \mathcal{Z}=\mathcal{X}(\mathcal{T}-F)^{-1} \mathcal{Z} \quad \text { and } \quad x_{0}=\mathbf{0} .
$$

Since the inward and outward throughflows at each subcompartment are the same at steady state, we have

$$
\tau=\check{\tau}=\hat{\tau} \quad \text { and } \quad T=\check{T}=\hat{T} .
$$

The static subthroughflow matrices, $\check{T}$ and $\hat{T}$, and the initial subthroughflow vector, $\tau_{0}$, can then be expressed as

$$
\begin{aligned}
\check{T} & =\mathcal{Z}+F \mathcal{X}^{-1} X, \\
\hat{T} & =\left(\mathcal{Y}+\operatorname{diag}\left(F^{T} \mathbf{1}\right)\right) \mathcal{X}^{-1} X=\mathcal{T} \mathcal{X}^{-1} X=\mathcal{R}^{-1} X, \\
\tau_{0} & =\check{\tau}_{0}=\hat{\tau}_{0}=\mathbf{0},
\end{aligned}
$$

similar to Eq. 2.11. Therefore, the static system partitioning methodology yields the subthroughflow and substorage matrices, $T$ and $X$, formulated in Eqs. 2.13 and 2.15. They quantify the distribution of environmental inputs and the organization of the associated storages generated by the inputs within the system. More specifically, the $(i, k)$-elements of these matrices, $\tau_{i_{k}}$ and $x_{i_{k}}$, are the throughflow at and storage in compartment $i$, generated by the environmental input into compartment $k, z_{k}$.

The system partitioning methodology introduced in this section is based on environmental inputs. The output-oriented system analysis is formulated in parallel, and the duality of the input- and output-oriented analysis is demonstrated through similarity relationships in Appendix F.

2.3. Subsystem scaling. Many current measures and indices of the static ecological network analysis are defined based on two constant matrix measures: $S$ and $N$. These matrices are considered as linear transformations that map input vector $z$ into throughflow and storage vectors, $\tau$ and $x$, respectively. That is,

$$
x=S z \quad \text { and } \quad \tau=N z .
$$


The elements of $S$ and $N, s_{i_{k}}$ and $n_{i_{k}}$, represent the storage and throughflow generated at compartment $i$ by unit environmental input into compartment $k[18,43]$.

In the proposed static methodology, the storage and throughflow vectors are directly formulated through the substorage and subthroughflow matrices introduced in the previous section:

$$
x=X \mathbf{1} \text { and } \tau=T \mathbf{1} .
$$

Therefore, in this new setting, $S$ and $N$ matrices are redundant in finding $x$ and $\tau$ vectors. Nevertheless, we provide alternative derivations for $S$ and $N$ below for completeness of the proposed theory and for other uses of these matrix measures introduced in the present paper. It will also be shown that, although the derivation rationale is different, some of the proposed static measures are equivalent to existing ones in the literature.

In order to analyze system behavior per unit input at steady state, all but the initial subsystems can be scaled by their corresponding positive environmental input, $z_{k}>0$ ( $\mathcal{Z}$ is invertible). Through the proposed methodology, using Eqs. 2.13 and 2.15, $S$ and $N$ can be defined as scaled substorage and subthroughflow matrices. That is,

$$
\begin{aligned}
S & =X \mathcal{Z}^{-1}, \\
\check{N} & =\check{T} \mathcal{Z}^{-1}=I+F \mathcal{X}^{-1} X \mathcal{Z}^{-1}, \\
\hat{N} & =\hat{T} \mathcal{Z}^{-1}=\left(\mathcal{Y}+\operatorname{diag}\left(F^{T} \mathbf{1}\right)\right) \mathcal{X}^{-1} X \mathcal{Z}^{-1},
\end{aligned}
$$

where $I$ is the $n \times n$ identity matrix. Componentwise, they are $S_{i k}=s_{i_{k}}=x_{i_{k}} / z_{k}$, $\check{N}_{i k}=\check{n}_{i_{k}}=\check{\tau}_{i_{k}} / z_{k}$, and $\hat{N}_{i k}=\hat{n}_{i_{k}}=\hat{\tau}_{i_{k}} / z_{k}$. Note that the physical dimension of $S$ is time [t], and both $\check{N}$ and $\hat{N}$ are dimensionless. As immediate consequences of these definitions, we have

$$
X=S \mathcal{Z} \quad \Rightarrow \quad x=S z,
$$

and, since the relationship $T=\check{T}=\hat{T}$ implies that $N=\check{N}=\hat{N}$,

$$
T=N \mathcal{Z} \quad \Rightarrow \quad \tau=N z .
$$

These relationships refine and extend the static network analysis and the static relationships given in Eq. 2.16 from the current compartmental level to the subcompartmental level.

Since Eq. 2.15 implies that $\mathcal{T}^{-1} T=\mathcal{X}^{-1} X, T$ can be expressed only in terms of system flows as

$$
T=\mathcal{Z}+F \mathcal{T}^{-1} T \quad \Rightarrow \quad T=\left(I-F \mathcal{T}^{-1}\right)^{-1} \mathcal{Z} .
$$

The scaled throughflow matrix $N$ defined in Eq. 2.18 can then be expressed in the following various forms using the relationships in Eqs. 2.15 and 2.21:

$$
N=T \mathcal{Z}^{-1}=I+Q^{x} S=\mathcal{R}^{-1} S=\left(I-Q^{\tau}\right)^{-1}
$$

where $Q^{\tau}=F \mathcal{T}^{-1}$, as defined in Section 2.4. The solution for the substorage matrix given in Eq. 2.13 also implies that the scaled substorage matrix defined in Eq. 2.18 can be expressed as

$$
S=X \mathcal{Z}^{-1}=-A^{-1}=\mathcal{X}(\mathcal{T}-F)^{-1} .
$$


Although derived with a different rationale, the last equalities derived for scaled substorage and subthroughflow matrices in Eqs. 2.22 and 2.23 are equivalent to those of the environ analysis [33]. The formulation of $S$ in Eq. 2.23 can further be modified into the following:

$$
S=\left(\mathcal{T} \mathcal{X}^{-1}-F \mathcal{X}^{-1}\right)^{-1}=\left(\mathcal{R}^{-1}-Q^{x}\right)^{-1}=\mathcal{R}\left(I-Q^{\tau}\right)^{-1}=(I-L)^{-1} \mathcal{R}
$$

where $L=\mathcal{R} Q^{x}$, as defined in Section 2.4. It is worth noting that, since the environmental input cancels out in the subsequent formulations of $S$ and $N$ due to the subsystem scaling under the assumption of the invertibility of $\mathcal{Z}$, the resultant formulas are independent of $\mathcal{Z}$. Due to linearity, these static formulations can still be used even if some environmental inputs are zero, for example, to compute $X$ and $T$ in Eqs. 2.19 and 2.20.

Using the relationship formulated in Eq. A.5, $S$ can alternatively be expressed for linear systems with constant environmental input at steady state as follows:

$$
S=\lim _{t \rightarrow \infty} S(t)=\Omega \lim _{t \rightarrow \infty}\left(\int_{t_{0}}^{t} \mathrm{e}^{(t-s) \Lambda} d s\right) \Omega^{-1}
$$

where the matrices $\Omega$ and $\Lambda$ are the eigenvector and eigenvalue matrices of the invertible diagonalizable flow intensity matrix $A$.

The matrix measures, $S$ and $N$, are treated separately in the environ theory [18]. The proposed methodology, however, unveils a unifying relationship that integrally combines these two matrix measures. Based on the subsystem scaling argument presented above, Eq. 2.15 implies that

$$
T=\mathcal{R}^{-1} X \Rightarrow S=\mathcal{R} N
$$

Therefore, these matrix measures are related in a unique and natural way in the sense that the storage distribution, $S$, generated by the throughflow distribution per unit input, $N$, is controlled by the residence time factor, $\mathcal{R}$.

The output-oriented subsystem scaling is introduced in the Supplementary Materials (Section S4.1).

2.4. Subsystem partitioning methodology. The dynamic subsystem partitioning methodology introduced by $[10,12]$ is summarized in the Supplementary Materials (Section S2). The subsystem partitioning methodology enables further decomposition or segmentation of subsystems dynamically along a given set of mutually exclusive and exhaustive subflow paths. This partitioning is similar to the dynamic system partitioning based on environmental inputs as summarized in Section 2.1. In this section, the static version of this dynamic subsystem partitioning will be introduced.

The static subsystem partitioning methodology decomposes composite intercompartmental flows and the associated storages generated by these flows into subflow and substorage segments along given subflow paths within a subsystem. Therefore, as the proposed system partitioning methodology formulates the distribution of environmental inputs and the organization of associated storages within the system, the subsystem partitioning formulates the distribution of intercompartmental subflows and the organization of associated substorages within the subsystems. The proposed mathematical method, therefore, as a whole, yields the decomposition of the system flows and storages to the utmost level or their "atomization." 


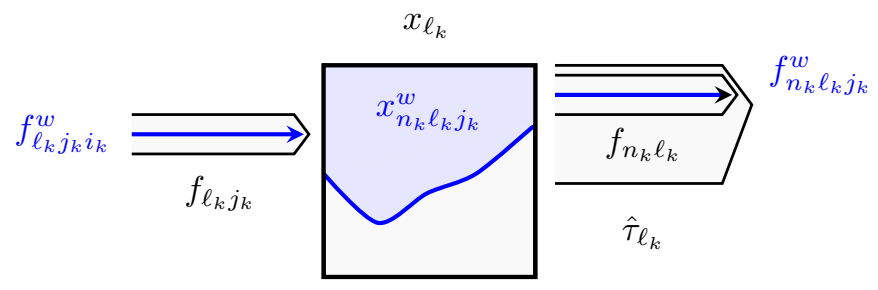

FIG. 3. Schematic representation of the subsystem partitioning. The transient inflow and outflow, $f_{\ell_{k} j_{k} i_{k}}^{w}$ and $f_{n_{k} \ell_{k} j_{k}}^{w}$, and associated substorage, $x_{n_{k} \ell_{k} j_{k}}^{w}$, at subcompartment $\ell_{k}$ along subflow path $p_{n_{k} j_{k}}^{w}=i_{k} \mapsto j_{k} \rightarrow \ell_{k} \rightarrow n_{k}$.

The static subsystem partitioning methodology will be formulated below using the directed subflow path terminology, which is adopted from the recent papers by $[10,12]$ (see Supplementary Materials, Section S2.1). The subsystems can further be decomposed into subflows and associated substorages along a set of mutually exclusive and exhaustive directed subflow paths. By mutually exclusive subflow paths, we mean that no given subflow path in a subsystem is a subpath, that is, completely inside of another path in the same subsystem. The exhaustiveness, in this context, means that such mutually exclusive subflow paths all together sum to the entire subsystem so partitioned.

Everything flows!.. - Heraclitus (535-475 BC)

2.4.1. Transient flows and storages. As indicated in this famous dictum, flows are one of the most important physical phenomena of existence. In this section, we introduce the transient subflows and the associated substorages generated by these flows.

Along a given subflow path $p_{n_{k} j_{k}}^{w}=i_{k} \mapsto j_{k} \rightarrow \ell_{k} \rightarrow n_{k}$, the transient inflow at subcompartment $\ell_{k}, f_{\ell_{k} j_{k} i_{k}}^{w}$, generated by the local input from $i_{k}$ into $j_{k}$ is the input segment that is transmitted from $j_{k}$ to $\ell_{k}$. Similarly, the transient outflow generated by the transient inflow at $\ell_{k}, f_{n_{k} \ell_{k} j_{k}}^{w}$, is the inflow segment that is transmitted from $\ell_{k}$ to the next subcompartment, $n_{k}$, along the path. The associated transient substorage in subcompartment $\ell_{k}, x_{n_{k} \ell_{k} j_{k}}^{w}$, is then the substorage segment derived from the transient inflow. The transient outflow at and substorage in subcompartment $\ell_{k}$ along subflow path $p_{n_{k} i_{k}}^{w}$ can be formulated as follows:

$$
x_{n_{k} \ell_{k} j_{k}}^{w}=\frac{x_{\ell}}{\tau_{\ell}} f_{\ell_{k} j_{k} i_{k}}^{w} \quad \text { and } \quad f_{n_{k} \ell_{k} j_{k}}^{w}=\frac{f_{n \ell}}{x_{\ell}} x_{n_{k} \ell_{k} j_{k}}^{w}=\frac{f_{n \ell}}{\tau_{\ell}} f_{\ell_{k} j_{k} i_{k}}^{w}
$$

based on the dynamic formulation introduced by [10] (see Fig. 3 and the Supplementary Materials, Section S2.3).

The equivalence of the flow and subflow, as well as the outward throughflow and subthroughflow intensities in the same direction can be expressed as

$$
q_{n \ell}^{x}=\frac{f_{n \ell}}{x_{\ell}}=\frac{f_{n_{k} \ell_{k}}}{x_{\ell_{k}}} \quad \text { and } \quad \frac{\tau_{\ell_{k}}}{x_{\ell_{k}}}=\frac{\tau_{\ell}}{x_{\ell}}
$$

for $k, \ell, n=1, \ldots, n$, due to Eqs. 2.5 and 2.15 [12]. These equivalences and Eq. B.5 imply that

$$
q_{n \ell}^{\tau}=\frac{f_{n \ell}}{\tau_{\ell}}=\frac{f_{n_{k} \ell_{k}}}{\tau_{\ell_{k}}} \quad \text { and } \quad r_{\ell}=\frac{x_{\ell}}{\tau_{\ell}}=\frac{x_{\ell_{k}}}{\tau_{\ell_{k}}} .
$$


Because $r_{\ell}, q_{n \ell}^{x}$, and $q_{n \ell}^{\tau}$ can all be expressed at both compartmental and subcompartmental levels, the subsystem partitioning is actually independent of the system partitioning. That is, the same analysis can be done along flow paths within the system, instead of subflow paths within the subsystems. This allows the flexibility of tracking arbitrary intercompartmental flows and storages generated by all or individual environmental inputs within the system. The governing Eq. 2.27 establishes the foundation of the static subsystem partitioning (see Fig. 3).

The factor $r_{\ell}$ in Eq. 2.27 measures how long the transient storage derived from the transient inflow stay in subcompartment $\ell_{k}$. The time required for the generation of a certain amount of storage in a compartment and the discharge of it from the compartment are the same at steady state. Consequently, $r_{\ell}$ will be called the exposure or residence time for compartment $\ell$. The flow intensities per unit storage and throughflow, $q_{n \ell}^{x}$ and $q_{n \ell}^{\tau}$, control the transient outflow emanates from the transient storage and that generated by the transient inflow, respectively, at subcompartment $\ell_{k}$. The factor $q_{n \ell}^{\tau}$ can also be interpreted as the fraction of transient inflow transmitted to the next step, that is, the next subcompartment along the path. The factors $q_{n \ell}^{x}$ and $q_{n \ell}^{\tau}$ will, therefore, be called the flow distribution factors from compartment $\ell$ to $n$ for transient storages and inflows, respectively.

The corresponding matrix measures $\mathcal{R}=\operatorname{diag}\left(\left[r_{1}, \ldots, r_{n}\right]\right), Q^{x}=\left(q_{n \ell}^{x}\right)$, and $Q^{\tau}=\left(q_{n \ell}^{\tau}\right)$ can be expressed as

$$
\mathcal{R}=\mathcal{X} \mathcal{T}^{-1}, \quad Q^{x}=F \mathcal{X}^{-1}, \quad \text { and } \quad Q^{\tau}=F \mathcal{T}^{-1}
$$

This implies that $Q^{\tau}=Q^{x} \mathcal{R}$. As their formulations indicate, $Q^{x}$ and $Q^{\tau}$ represent intercompartmental flow intensities per unit storage and throughflow, respectively, for the entire system. Based on the discussion above, the matrix measure $\mathcal{R}$ will be called the residence time or storage distribution matrix for transient inflows, and $Q^{x}$ and $Q^{\tau}$ will be called the intercompartmental flow intensity per unit storage and throughflow or flow distribution matrices for transient storages and inflows, respectively, depending on the context. Note that, sometimes $Q^{\tau}$ is called transfer coefficients, technical coefficients in economics, or stoichiometric coefficients in chemistry. The unit of $\mathcal{R}$ is time, [t], that of $Q^{x}$ is inverse time, [ $\left.\mathrm{t}^{-1}\right]$, and $Q^{\tau}$ is dimensionless.

The transient flows and storages indirectly along a given particular flow path can also be computed using Eq. 2.27. More specifically, along subflow path $p_{v_{k} j_{k}}^{w}=$ $i_{k} \mapsto j_{k} \rightsquigarrow \ell_{k} \rightsquigarrow \cdots \rightsquigarrow u_{k} \rightarrow v_{k}$, the transient subflow into subcompartment $v_{k}, \tilde{\tau}_{v_{k}}^{w}$, generated by the local input of the path, $\hat{\tau}_{i_{k}}^{w}$, and the associated transient substorage generated by this subflow, $x_{v_{k}}^{w}$, can be formulated as

$$
x_{v_{k}}^{w}=r_{v} \check{\tau}_{v_{k}}^{w} \quad \text { where } \quad \check{\tau}_{v_{k}}^{w}=q_{v u}^{\tau} \cdots q_{\ell j}^{\tau} \hat{\tau}_{i_{k}}^{w} .
$$

The equation for $\check{\tau}_{v_{k}}^{w}$ above is similar to a procedure suggested by [23].

The natural subsystem partitioning is defined as the set of mutually exclusive and exhaustive subflow paths whose local inputs and outputs, except for the closed paths, are environmental inputs and outputs, respectively (see Supplementary Materials, Section S2.1). Let $T_{\ell k}^{(m)}=\tau_{\ell_{k}}^{(m)}$ and $X_{\ell k}^{(m)}=x_{\ell_{k}}^{(m)}$ be the transient subthroughflow and associated substorage matrices generated at step $m$ along all possible subflow paths of the natural subsystem partitioning. Componentwise, we have

$$
x_{\ell_{k}}^{(m)}=\sum_{\ell=1}^{n} r_{\ell} \tau_{\ell_{k}}^{(m)} \quad \text { and } \quad \tau_{n_{k}}^{(m+1)}=\sum_{\ell=1}^{n} q_{n \ell}^{\tau} \tau_{\ell_{k}}^{(m)}=\sum_{\ell=1}^{n} q_{n \ell}^{x} x_{\ell_{k}}^{(m)},
$$


due to Eq. 2.27. Note that, the outward throughflows generated at step $(m)$ are the inward throughflows at the next step, $(m+1)$. In matrix form, these equations can be expressed as

$$
X^{(m)}=\mathcal{R} T^{(m)} \quad \text { and } \quad T^{(m+1)}=Q^{\tau} T^{(m)}=Q^{x} X^{(m)} .
$$

Equations 2.31 and 2.33 formulate transient flows and associated storages along a particular pathway and all possible pathways of length $(m)$, respectively. The relationships in Eq. 2.33 imply also that

$$
X^{(m+1)}=L X^{(m)} \quad \text { where } \quad L=\mathcal{R} Q^{x} .
$$

This dimensionless matrix $L$ will be called the storage distribution matrix for transient storages.

It is worth noting that, using Eq. 2.33 with $X^{(m)}=X$ and $T^{(m)}=T$, Eq. 2.15 can be written in terms of distribution matrices:

$$
X=\mathcal{R} T \quad \text { and } \quad \tilde{T}=F D=Q^{\tau} T=Q^{x} X .
$$

The decomposition matrix, D, is defined in Eq. B.4. Here $\tilde{T}=\left(\tilde{\tau}_{i_{k}}\right)$ will be called the intercompartmental subthroughflow matrix and defined as $\tilde{T}=T-\mathcal{Z}$, which, componentwise, reads $\tilde{\tau}_{i_{k}}=\tau_{i_{k}}-z_{i_{k}}$.

Using the distribution matrices, the matrix measures $S, N, X$, and $T$ can be expanded as infinite geometric series as shown in Appendix D:

$$
N=\sum_{m=0}^{\infty}\left(Q^{\tau}\right)^{m} \quad \text { and } \quad S=\left(\sum_{m=0}^{\infty} L^{m}\right) \mathcal{R} .
$$

These geometric series expansions of the subthroughflow and substorage matrices integrate the proposed system and subsystem partitioning methodologies.

The matrix $S$ is expressed as the infinite sum of the storage distribution matrix $L$ in Eq. 2.35. As suggested by this formulation, $S$ will be called the cumulative storage distribution matrix. Similar to the diagonal elements of $\mathcal{R}, r_{i}=x_{i} / \tau_{i}$, the $(i, k)$-element of $S, s_{i_{k}}=x_{i_{k}} / z_{k}$, can be interpreted as the residence time in compartment $i$ of the storage generated by the unit environmental input into $k$. Therefore, $S$ and $\mathcal{R}$ will, alternatively, be called the simple and composite residence time matrices, respectively. As suggested by Eq. $2.35, N$ will be called the cumulative flow distribution matrix. Likewise, similar to the nondimensional $(i, k)$-element of $Q^{\tau}$, $q_{i k}^{\tau}=f_{i k} / \tau_{k}$, that of $N, n_{i_{k}}=\tau_{i_{k}} / z_{k}$, can be interpreted as the fraction/multiple of unit environmental input into compartment $k$ that is distributed to $i$.

The geometric series expansion of $S$ and $N$, as formulated in Eq. 2.35, were also proposed in the literature with different derivations and interpretations $[22,18,16]$. Instead of the diagonal matrix $\mathcal{R}$ in the proposed formulation for $S$, however, the authors used a scalar time interval $\Delta t \in[0,1]$ through dimension analysis, which cannot describe system dynamics accurately. Moreover, the indirect flow contributions were defined as the flow contributions carried by subsequent steps after their first entrance into a compartment. Therefore, the higher-powered terms $(m>1)$ in the series expansions are considered as indirect contributions in these formulations [37, $39,22,48,5,32]$. Even the direct transactions between compartments after the first step $(m=1)$ are counted as indirect. Unlike the physical and measurable indirect flow and storage formulations proposed in the next section, the ones proposed in the 
literature are microscopic quantities and, therefore, cannot accurately describe the indirect transactions.

The output-oriented subsystem partitioning methodology can be formulated similar to the input-oriented subsystem partitioning as presented in the Supplementary Materials (Section S4.2).

2.5. Quantitative analysis of food webs. Flow of energy and matter through ecosystems is one of the main topics of ecosystem ecology. The proposed methodology is particularly designed for quantitative analysis of ecosystem flows and storages. The method is applicable to both naturogenic and anthropogenic ecological models. The physical terminology reflecting this generality and wide applicability of the proposed method can be adjusted depending on specific ecological models of interest.

One of the immediate potential ecological applications of the method is the quantitative analysis of food chains and food webs. In the context of the proposed methodology, a food web can be considered as a naturogenic ecosystem, the system compartments represent species in the food web, the conserved quantity in question becomes nutrient or energy, and flow paths correspond to food chains. All formulations and relationships introduced in physical terms in the present paper are applicable to food webs and can, therefore, be translated to the food web terminology using these and similar correspondences and can be interpreted accordingly.

In this setting, the nonnegative $(i, k)$-element, $f_{i k}$, of the direct flow matrix, $F$, for example, represents an interspecific interaction between species $i$ and $k$ and measures the rate of nutrient or energy flow from species $k$ in a lower trophic level to $i$ in the next level due to trophic interactions, such as predation. In other words, for nutrient being the conserved quantity, $f_{i k}$ represents the amount of prey $k$ consumed by predator $i$ per unit time. Nutrient or energy stored in a species through all trophic interactions is represented by $x_{i}$. The proposed system partitioning methodology, then, decomposes system flows and storages in a way that the fate of environmental nutrient or energy input into any species can be tracked individually and separately throughout the food web. More specifically, the $(i, k)$-elements of the subthroughflow and substorage matrices defined in Eqs. 2.13 and 2.15, $\tau_{i_{k}}$ and $x_{i_{k}}$, represent the nutrient or energy flow segment at and storage portion in species $i$ derived through interspecific interactions from environmental input into species $k$. The output-oriented subthroughflow and substorage defined in Eqs. F.6 and F.7, $\bar{\tau}_{i_{k}}$ and $\bar{x}_{i_{k}}$, then represent the nutrient or energy flow segment at and storage portion in species $i$ destined to leave the food web as unusable waste or dissipation via species $k$. In that sense, the input and output-oriented analyses concern bottom-up and top-down activities, respectively.

The proposed subsystem partitioning methodology enables further analysis of nutrient or energy flow and storage transfers through trophic interactions along arbitrary food chains within complex food webs. The transient flows and storages formulated in Eqs. 2.31 and 2.33 determine the nutrient or energy transfer along a particular and all possible food chains of arbitrary length, respectively. Therefore, the spread of the arbitrary amount of nutrient or energy from one species to the entire network along all possible food chains can be monitored. Moreover, while the input-oriented analysis enables the ascertainment of nutrient or energy generation in each species along a particular food chain, the output-oriented analysis allows for the determination of unusable waste and energy dissipation in each species along the chain.

The direct, indirect, cycling, acyclic, and total (diact) flows and storages introduced in the next section, then, quantify the diact interspecific interactions and 
influence of one compartment directly and indirectly on any other in the food web. Therefore, these measures can also be used for the quantitative analysis of food webs. A quantitative binary characterization and classification of interspecific interactions is proposed in Section 2.7.

2.6. The diact flows and storages. In this section, we introduce five important transaction types for static ecological systems based on the partitioning methodologies: the diact flows and storages. The indirect flows (denoted by $i$ ) will be formulated below, and parallel derivations for transfer (t), direct (d), cycling (c), and acyclic (a) flows will be presented in Appendix E.

The dynamic diact flows and storages have been introduced by [10, 12] based on the subsystem partitioning methodology using subflow paths. The static diact transactions are formulated through this path-based approach in the Supplementary Materials (Section S3.1). In this section, the static diact flows and storages are defined using an alternative approach. This static approach is more practical but designed for only static cases whereas the path-based approach works for both static and dynamic systems.

The composite transfer flow will be defined as the total intercompartmental transient flow from one compartment, directly or indirectly through other compartments, to another. The composite direct, indirect, acyclic, and cycling flows from the initial compartment to the terminal compartment are then defined as the direct, indirect, non-cycling, and cycling segments at the terminal compartment of the composite transfer flow (see Fig. 4). The simple transfer flow will also be defined as the total intercompartmental transient flow from an input-receiving subcompartment, directly or indirectly through other compartments, to another subcompartment. The simple direct, indirect, acyclic, and cycling flows from the initial input-receiving subcompartment to the terminal subcompartment are then defined as the direct, indirect, non-cycling, and cycling segments at the terminal subcompartment of the simple transfer flow (see Fig. 4). The associated simple and composite diact storages are defined as the storages generated by the corresponding diact flows. The simple and composite diact flows and storages at both subcompartmental and compartmental levels are formulated below and in Appendix E.

The simple indirect subflow from an input-receiving subcompartment $k_{k}$ to $i_{k}$ in the $k^{t h}$ subsystem can be formulated as follows:

$$
\tau_{i_{k} k_{k}}^{\mathrm{i}}=\sum_{\substack{j=1 \\ j \neq k}}^{n} f_{i_{k} j_{k}}=\check{\tau}_{i_{k}}-z_{i_{k}}-f_{i_{k} k_{k}}
$$

for $i, k=1, \ldots, n$. The proportionality introduced in Eq. E.3 implies that the composite indirect subflow from subcompartment $k_{\ell}$ to $i_{\ell}, \tau_{i_{\ell} k_{\ell}}^{\mathrm{i}}$, which is a subflow parallel to $\tau_{i_{k} k_{k}}^{\mathrm{i}}$ in the $\ell^{\text {th }}$ subsystem, can then be formulated as

$$
\tau_{i_{\ell} k_{\ell}}^{\mathrm{i}}=\tau_{i_{k} k_{k}}^{\mathrm{i}} \frac{\check{\tau}_{k_{\ell}}}{\check{\tau}_{k_{k}}}=\left(\check{\tau}_{i_{k}}-z_{i_{k}}-f_{i_{k} k_{k}}\right) \frac{\check{\tau}_{k_{\ell}}}{\check{\tau}_{k_{k}}} .
$$

We can drop the in and out accents as $\check{\tau}=\hat{\tau}$ at steady state. Therefore, the composite indirect flow from compartment $k$ to $i$ becomes

$$
\tau_{i k}^{\mathrm{i}}=\sum_{\ell=1}^{n} \tau_{i_{\ell} k_{\ell}}^{\mathrm{i}}=\sum_{\ell=1}^{n}\left(\tau_{i_{k}}-z_{i_{k}}-f_{i_{k} k_{k}}\right) \frac{\tau_{k_{\ell}}}{\tau_{k_{k}}}=n_{i k}^{\mathrm{i}} \sum_{\ell=1}^{n} \tau_{k_{\ell}}=n_{i k}^{\mathrm{i}} \tau_{k}
$$




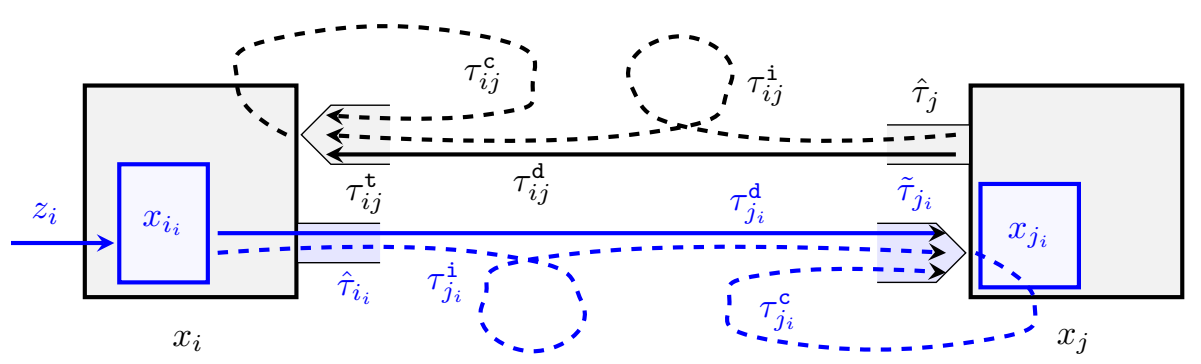

FIG. 4. Schematic representation of the composite and simple diact flows. Solid arrows represent direct flows, and dashed arrows represent indirect flows through other compartments (not shown). The composite diact flows (black) generated by outward throughflow $\hat{\tau}_{j}$ (i.e. derived from all environmental inputs): direct flow, $\tau_{i j}^{d}=f_{i j}$, indirect flow, $\tau_{i j}^{i}$, acyclic flow, $\tau_{i j}^{a}=\tau_{i j}^{t}-\tau_{i j}^{c}$, cycling flow, $\tau_{i j}^{c}$, and transfer flow, $\tau_{i j}^{t}$. The simple diact flows (blue) generated by outward subthroughflow $\hat{\tau}_{i_{i}}$ (i.e. derived from single environmental input $z_{i}$ ): direct flow, $\tau_{j_{i}}^{d}=\tau_{j_{i} i_{i}}^{d}=f_{j_{i} i_{i}}$, indirect flow, $\tau_{j_{i}}^{i}=\tau_{j_{i} i_{i}}^{i}$, acyclic flow, $\tau_{j_{i}}^{a}=\tau_{j_{i} i_{i}}^{a}=\tilde{\tau}_{j_{i}}-\tau_{j_{i}}^{c}$, cycling flow, $\tau_{j_{i}}^{c}=\tau_{j_{i} i_{i}}^{c}$, and transfer flow, $\tau_{j_{i}}^{t}=\tilde{\tau}_{j_{i}}=\check{\tau}_{j_{i}}-z_{j_{i}}$. Note that the cycling flows at the terminal (sub)compartment may include the segments of the direct and/or indirect flows at that (sub)compartment, if the cycling flows indirectly pass through the corresponding initial (sub)compartment (see Fig. 10). Therefore, the acyclic flows are composed of the segments of the direct and/or indirect flows.

where $\tau_{k}=\sum_{\ell=1}^{n} \tau_{k_{\ell}}$

$$
n_{i k}^{\mathrm{i}}=\frac{\tau_{i_{k}}-z_{i_{k}}-f_{i_{k} k_{k}}}{\tau_{k_{k}}}=\frac{n_{i_{k}}-\delta_{i k}}{n_{k_{k}}}-\frac{f_{i k}}{\tau_{k}},
$$

and $n_{i_{k}}$ is the $(i, k)$-element of $N$ defined in Section 2.3. The factor $n_{i k}^{\mathrm{i}}$ will be called the indirect flow distribution factor. The second equality, which is derived from Eqs. B.5 and B.7, can be used when there is a zero environmental input and, therefore, $\tau_{k_{k}}=0$ for some $k$, as discussed in Section 2.3. The indirect flow distribution matrix, $N^{\mathrm{i}}=\left(n_{i k}^{\mathrm{i}}\right)$, can then be formulated as

$$
N^{\mathrm{i}}=(N-I) \mathcal{N}^{-1}-F \mathcal{T}^{-1}
$$

where $\mathcal{N}=\operatorname{diag}(N)$. The proportionality introduced in Eq. E.3 implies also that

$$
x_{i_{\ell} k_{\ell}}^{\mathrm{i}}=\tau_{i_{\ell} k_{\ell}}^{\mathrm{i}} \frac{x_{i}}{\tau_{i}} \quad \text { and } \quad x_{i k}^{\mathrm{i}}=\sum_{\ell=1}^{n} x_{i_{\ell} k_{\ell}}^{\mathrm{i}}=\tau_{i k}^{\mathrm{i}} \frac{x_{i}}{\tau_{i}}
$$

which is consistent with the subsystem partitioning formulated in Eq. 2.27. Therefore, the $\ell^{\text {th }}$ composite indirect subflow and associated substorage matrices, $\mathcal{T}_{\ell}^{\mathrm{i}}=\left(\tau_{i_{\ell} k_{\ell}}^{\mathrm{i}}\right)$ and $\mathcal{X}_{\ell}^{\mathrm{i}}=\left(x_{i_{\ell} k_{\ell}}^{\mathrm{i}}\right)$, can be expressed in matrix form as follows:

$$
T_{\ell}^{\mathrm{i}}=N^{\mathrm{i}} \mathcal{T}_{\ell}=(N-I) \mathcal{N}^{-1} \mathcal{T}_{\ell}-F \mathcal{T}^{-1} \mathcal{T}_{\ell} \quad \text { and } \quad X_{\ell}^{\mathrm{i}}=S^{\mathrm{i}} \mathcal{T}_{\ell}=\mathcal{R} T_{\ell}^{\mathrm{i}} .
$$

The matrix $S^{\mathrm{i}}=\left(s_{i k}^{\mathrm{i}}\right)$ defined as

$$
s_{i k}^{\mathrm{i}}=r_{i} n_{i k}^{\mathrm{i}} \quad \Rightarrow \quad S^{\mathrm{i}}=\mathcal{R} N^{\mathrm{i}}
$$

will be called the indirect storage distribution matrix. Due to Eq. 2.38, the composite indirect flow and associated storage matrices, $T^{\mathrm{i}}=\left(\tau_{i k}^{\mathrm{i}}\right)$ and $X^{\mathrm{i}}=\left(x_{i k}^{\mathrm{i}}\right)$, then become

$$
T^{\mathrm{i}}=N^{\mathrm{i}} \mathcal{T}=(N-I) \mathcal{N}^{-1} \mathcal{T}-F \quad \text { and } \quad X^{\mathrm{i}}=S^{\mathrm{i}} \mathcal{T}=\mathcal{R} T^{\mathrm{i}} .
$$


The composite indirect throughflow and compartmental storage matrices and vectors can also be formulated as

$$
\mathcal{T}^{\mathrm{i}}=\operatorname{diag}\left(T^{\mathrm{i}} \mathbf{1}\right) \Rightarrow \tau^{\mathrm{i}}=\mathcal{T}^{\mathrm{i}} \mathbf{1} \quad \text { and } \quad \mathcal{X}^{\mathrm{i}}=\operatorname{diag}\left(X^{\mathrm{i}} \mathbf{1}\right) \Rightarrow x^{\mathrm{i}}=\mathcal{X}^{\mathrm{i}} \mathbf{1} .
$$

The simple indirect flows and storages can be expressed in terms of the composite indirect flows and storages as follows:

$$
\tau_{i_{k}}^{\mathrm{i}}=\tau_{i_{k} k_{k}}^{\mathrm{i}} \quad \text { and } \quad x_{i_{k}}^{\mathrm{i}}=x_{i_{k} k_{k}}^{\mathrm{i}} .
$$

Let $\mathrm{T}$ be a diagonal matrix whose diagonal elements are the diagonal elements of the subthroughflow matrix, that is $\mathrm{T}=\operatorname{diag}(T)$. The simple indirect flow and storage matrices, $\tilde{T}^{\mathrm{i}}=\left(\tau_{i_{k}}^{\mathrm{i}}\right)$ and $\tilde{X}^{\mathrm{i}}=\left(x_{i_{k}}^{\mathrm{i}}\right)$, can then be formulated as

$$
\tilde{T}^{\mathrm{i}}=N^{\mathrm{i}} \mathrm{T} \quad \text { and } \quad \tilde{X}^{\mathrm{i}}=S^{\mathrm{i}} \mathrm{T}=\mathcal{R} \tilde{T}^{\mathrm{i}} .
$$

The $k^{\text {th }}$ simple indirect flow and storage matrices, $\tilde{\mathcal{T}}_{k}^{\mathrm{i}}$ and $\tilde{\mathcal{X}}_{k}^{\mathrm{i}}$, are the diagonal matrices whose nonzero entries are the $k^{\text {th }}$ column vectors of $\tilde{T}^{\mathrm{i}}$ and $\tilde{X}^{\mathrm{i}}$, respectively. The other simple diact flow and storage matrices can also be formulated similarly (see Appendix E).

The difference between the composite and simple indirect flows, $\tau_{i k}^{\mathrm{i}}$ and $\tau_{i_{k}}^{\mathrm{i}}$, and associated storages, $x_{i k}^{\dot{i}}$ and $x_{i_{k}}^{\dot{i}}$, is that the composite flow and storage from compartment $k$ to $i$ are generated by outward throughflow $\hat{\tau}_{k}$ derived from all environmental inputs, and their simple counterparts from input-receiving subcompartment $k_{k}$ to $i_{k}$ are generated by outward subthroughflow $\hat{\tau}_{k_{k}}$ derived from single environmental input $z_{k}$ (see Fig. 4). In that sense, the composite and simple diact flows and storages measure the influence of one compartment on another induced by all and a single environmental input, respectively. The same relationships are also valid between the other composite and simple diact flows and storages.

Since, due to their constructions, the simple indirect flows and storages are constituents of the corresponding composite indirect flows and storages, we have

$$
T^{i} \geq \tilde{T}^{i} \Rightarrow T^{i}=T^{i}-\tilde{T}^{i} \geq \mathbf{0} \quad \text { and } \quad X^{i} \geq \tilde{X}^{i} \Rightarrow \dot{X}^{i}=X^{i}-\tilde{X}^{i} \geq \mathbf{0} .
$$

These inequalities are defined componentwise. The elements of the $k^{\text {th }}$ columns of $T^{i}$ and $\dot{X}^{i}$ are the indirect flows and storages from compartment $k$ to the others generated by all environmental inputs but $z_{k}$ (see Fig. 4). The matrices $\dot{T}^{\mathrm{i}}$ and $\dot{X}^{\mathrm{i}}$ will accordingly be called complementary indirect flows and storages, respectively.

This static indirect flow and storage formulation significantly simplifies the pathbased dynamic approach introduced in the Supplementary Materials (Section S3.1), as there may be a large number of subflow paths from compartment $k$ to $i$ to consider. With the static approach introduced in this section, the indirect flows and storages as well as the other diact flows and storages are obtained by simple, one step computations formulated in Eqs. 2.38 and 2.41 or Eq. 2.44, in matrix form.

The composite direct flow and associated storage matrices are introduced in Appendix E. They are

$$
T^{\mathrm{d}}=F \quad \text { and } \quad X^{\mathrm{d}}=\mathcal{R} F .
$$

The corresponding direct flow and storage distribution matrices are formulated in terms of the transient flow and storage distribution matrices as

$$
N^{\mathrm{d}}=Q^{\tau} \quad \text { and } \quad S^{\mathrm{d}}=\mathcal{R} Q^{\tau} .
$$


TABLE 1

The input-oriented, flow-based diact flow and storage distribution and the simple and composite diact (sub)flow and (sub)storage matrices. The superscript $\left(^{*}\right)$ in each equation represents any of the diact symbols.

\begin{tabular}{|c|c|c|c|c|}
\hline diact & flow and storage distributio & matrices & flows & storages \\
\hline d & $N^{\mathrm{d}}=F \mathcal{T}^{-1}$ & & & \\
\hline$i$ & $N^{\mathrm{i}}=(N-I) \mathcal{N}^{-1}-F \mathcal{T}^{-1}$ & & $T^{*}=N^{*} \mathcal{T}$ & $X^{*}=S^{*} \mathcal{T}$ \\
\hline $\mathrm{a}$ & $N^{\mathrm{a}}=\left(\mathcal{N}^{-1} N-I\right) \mathcal{N}^{-1}$ & $S^{*}=\mathcal{R} N^{*}$ & $T_{\ell}^{*}=N^{*} \mathcal{T}_{\ell}$ & $X_{\ell}^{*}=S^{*} \mathcal{T}_{\ell}$ \\
\hline c & $N^{\mathrm{c}}=\left(N-\mathcal{N}^{-1} N\right) \mathcal{N}^{-1}$ & & $\tilde{T}^{*}=N^{*} \mathrm{~T}$ & $\tilde{X}^{*}=S^{*} \mathrm{~T}$ \\
\hline$t$ & $N^{\mathrm{t}}=(N-I) \mathcal{N}^{-1}$ & & & \\
\hline
\end{tabular}

There is a functional similarity between $T^{\mathrm{i}}$ and $T^{\mathrm{d}}=F$; the $(i, k)-$ element of $T^{\mathrm{i}}$, $\tau_{i k}^{\mathrm{i}}$, is the composite indirect flow, while that of $F, \tau_{i k}^{\mathrm{d}}=f_{i k}$, is the composite direct flow from compartment $k$ to $i$ (see Fig. 10). The simple direct flows and storages can also be formulated as follows:

$$
\tilde{T}^{\mathrm{d}}=N^{\mathrm{d}} \mathrm{T} \quad \text { and } \quad \tilde{X}^{\mathrm{d}}=S^{\mathrm{d}} \mathrm{T}=\mathcal{R} \tilde{T}^{\mathrm{d}} .
$$

The composite indirect flow, $\tau_{i j}^{\mathrm{i}}$, can be considered as the composite transfer (total) flow diminished by the composite direct flow from $j$ to $i$ (see Fig. 4). Therefore, it can be formulated as $\tau_{i j}^{\mathrm{i}}=\tau_{i j}^{\mathrm{t}}-f_{i j}$. Consequently, we have the following supplementary relationship:

$$
T^{\mathrm{t}}=T^{\mathrm{d}}+T^{\mathrm{i}} \quad \text { and } \quad X^{\mathrm{t}}=X^{\mathrm{d}}+X^{\mathrm{i}}
$$

where $T^{\mathrm{t}}$ and $X^{\mathrm{t}}$ will be called the composite transfer flow and associated storage matrices. The $(i, k)$-elements of $T^{\mathrm{t}}$ and $X^{\mathrm{t}}, \tau_{i k}^{\mathrm{t}}$ and $x_{i k}^{\mathrm{t}}$, are the composite transfer flow and storage from compartment $k$ directly or indirectly through other compartments to $i$. Comparing Eq. 2.52 with Eq. 2.44, the transfer flow and storage matrices can be expressed as follows:

$$
T^{\mathrm{t}}=N^{\mathrm{t}} \mathcal{T}=(N-I) \mathcal{N}^{-1} \mathcal{T} \quad \text { and } \quad X^{\mathrm{t}}=S^{\mathrm{t}} \mathcal{T}=\mathcal{R} T^{\mathrm{t}}
$$

where the corresponding transfer flow and storage distribution matrices are

$$
N^{\mathrm{t}}=(N-I) \mathcal{N}^{-1} \quad \text { and } \quad S^{\mathrm{t}}=\mathcal{R} N^{\mathrm{t}} .
$$

The simple transfer flows and storages can also be formulated as

$$
\tilde{T}^{\mathrm{t}}=N^{\mathrm{t}} \mathrm{T}=\tilde{T} \quad \text { and } \quad \tilde{X}^{\mathrm{t}}=S^{\mathrm{t}} \mathrm{T}=\mathcal{R} \tilde{T}^{\mathrm{t}}=\tilde{X},
$$

where $\tilde{X}=X-\mathcal{R} \mathcal{Z}$ will be called the intercompartmental substorage matrix. That is, the simple transfer flow and storage matrices are equal to the intercompartmental subthroughflow and substorage matrices. Note that, similar to Eq. 2.52, we have

$$
\tilde{T}^{\mathrm{t}}=\tilde{T}^{\mathrm{d}}+\tilde{T}^{\mathrm{i}} \quad \text { and } \quad \tilde{X}^{\mathrm{t}}=\tilde{X}^{\mathrm{d}}+\tilde{X}^{\mathrm{i}} .
$$

The composite cycling and acyclic flows from one compartment to another are defined as the cycling and non-cycling segments of the composite transfer flow at the terminal compartment. As detailed in Appendix E, the composite acyclic flow and storage matrices can be formulated as follows:

$$
T^{\mathrm{a}}=N^{\mathrm{a}} \mathcal{T}=\left(\mathcal{N}^{-1} N-I\right) \mathcal{N}^{-1} \mathcal{T} \quad \text { and } \quad X^{\mathrm{a}}=S^{\mathrm{a}} \mathcal{T}=\mathcal{R} T^{\mathrm{a}}
$$


where the corresponding acyclic flow and storage distribution matrices are

$$
N^{\mathrm{a}}=\left(\mathcal{N}^{-1} N-I\right) \mathcal{N}^{-1} \quad \text { and } \quad S^{\mathrm{a}}=\mathcal{R} N^{\mathrm{a}} .
$$

The simple acyclic flow and storage can then, respectively, be defined as

$$
\tilde{T}^{\mathrm{a}}=N^{\mathrm{a}} \mathrm{\top} \quad \text { and } \quad \tilde{X}^{\mathrm{a}}=S^{\mathrm{a}} \mathrm{T}=\mathcal{R} \tilde{T}^{\mathrm{a}} .
$$

By definition, the composite cycling and acyclic flows and storages are related as $\tau_{i j}^{\mathrm{t}}=\tau_{i j}^{\mathrm{c}}+\tau_{i j}^{\mathrm{a}}$ and $x_{i j}^{\mathrm{t}}=x_{i j}^{\mathrm{c}}+x_{i j}^{\mathrm{a}}$. In matrix form, we have the following supplementary relationship:

$$
T^{\mathrm{t}}=T^{\mathrm{c}}+T^{\mathrm{a}} \quad \text { and } \quad X^{\mathrm{t}}=X^{\mathrm{c}}+X^{\mathrm{a}},
$$

analogous to Eq. 2.52. Using these relationships, the composite cycling flow and storage matrices are derived in Appendix E as follows:

$$
T^{\mathrm{c}}=N^{\mathrm{c}} \mathcal{T}=\left(N-\mathcal{N}^{-1} N\right) \mathcal{N}^{-1} \mathcal{T} \quad \text { and } \quad X^{\mathrm{c}}=S^{\mathrm{c}} \mathcal{T}=\mathcal{R} T^{\mathrm{c}}
$$

where the corresponding cycling flow and storage distribution matrices are

$$
N^{\mathrm{c}}=\left(N-\mathcal{N}^{-1} N\right) \mathcal{N}^{-1} \text { and } S^{\mathrm{c}}=\mathcal{R} N^{\mathrm{c}} .
$$

The $(i, k)$-elements of $T^{\mathrm{c}}$ and $T^{\mathrm{a}}, \tau_{i k}^{\mathrm{c}}$ and $\tau_{i k}^{\mathrm{a}}$, are the cycling and non-cycling segments of the composite transfer flow at compartment $i$ from $k$. Those elements of $X^{\mathrm{c}}$ and $X^{\mathrm{a}}, x_{i k}^{\mathrm{c}}$ and $x_{i k}^{\mathrm{a}}$, are the storages generated by the corresponding composite cycling and acyclic flows, respectively. These cycling and acyclic flows and storages can, therefore, be interpreted as the flows and storages that visit compartment $i$ multiple times and only once, respectively, after being transmitted from compartment $k$. The simple cycling flow and storage can then be defined as follows:

$$
\tilde{T}^{\mathrm{c}}=N^{\mathrm{c}} \mathrm{T} \quad \text { and } \quad \tilde{X}^{\mathrm{c}}=S^{\mathrm{c}} \mathrm{T}=\mathcal{R} \tilde{T}^{\mathrm{c}} .
$$

Analogous to their composite counterparts, the simple cycling and acyclic flows and storages are related as $\tilde{\tau}_{i_{k}}=\tau_{i_{k}}^{\mathrm{c}}+\tau_{i_{k}}^{\mathrm{a}}$ and $\tilde{x}_{i_{k}}=x_{i_{k}}^{\mathrm{c}}+x_{i_{k}}^{\mathrm{a}}$. In matrix form, these relationships read

$$
\tilde{T}=\tilde{T}^{\mathrm{c}}+\tilde{T}^{\mathrm{a}} \text { and } \quad \tilde{X}=\tilde{X}^{\mathrm{c}}+\tilde{X}^{\mathrm{a}}
$$

similar to Eq. 2.60. The $(i, k)$-elements of $\tilde{T}^{\mathrm{a}}$ and $\tilde{T}^{\mathrm{c}}, \tau_{i_{k}}^{\mathrm{a}}$ and $\tau_{i_{k}}^{\mathrm{c}}$, represent the cycling and non-cycling segments of the intercompartmental subthroughflow at compartment $i$ from $k$ generated by environmental input $z_{k}$.

The composite cycling subflow from compartment $i$ into itself can be defined as reflexive transfer or indirect flow. The relationship between the corresponding flow distribution factors becomes

$$
n_{i i}^{\mathrm{c}}=\frac{n_{i_{i}}-1}{n_{i_{i}}}=n_{i i}^{\mathrm{i}}=n_{i i}^{\mathrm{t}} .
$$

This unifying relationship shows how the composite cycling and transfer or indirect flows, as well as their distribution factors are complementarily related (see Fig. 10). In matrix form, the relationship in terms of diact flows and storages can be expressed as follows:

$$
\operatorname{diag}\left(T^{\mathrm{c}}\right)=\operatorname{diag}\left(T^{\mathrm{t}}\right)=\operatorname{diag}\left(T^{\mathrm{i}}\right) \text { and } \operatorname{diag}\left(X^{\mathrm{c}}\right)=\operatorname{diag}\left(X^{\mathrm{t}}\right)=\operatorname{diag}\left(X^{\mathrm{i}}\right) .
$$


The same relationships can be formulated for the simple diact flows and storages.

The flow-based diact flow and storage distribution matrices, as well as the corresponding simple and composite diact flow and storage matrices are listed in Table 1. Through a dynamic approach similar to the static approach introduced above in this section, the dynamic diact flows and storages have recently been formulated explicitly by [10], as listed in the Supplementary Materials (Table S1).

The flow-based diact flows and storages listed in terms of $N$ in Table 1 can be expressed in terms of $S$ using the relationships given in Eqs. 2.26 and E.46, as formulated in Appendix E.6. The storage-based acyclic flow and storage distribution matrices, for example, become

$$
S^{\mathrm{a}, x}=\left(\mathcal{S}^{-1} S-I\right) \mathcal{S}^{-1} \quad \text { and } \quad N^{\mathrm{a}, x}=\mathcal{R} S^{\mathrm{a}, x} .
$$

Therefore,

$$
T^{\mathrm{a}}=S^{\mathrm{a}, x} \mathcal{X} \quad \text { and } \quad X^{\mathrm{a}}=N^{\mathrm{a}, x} \mathcal{X}
$$

All the other input-oriented, storage-based diact flow and storage distribution matrices, together with their corresponding flow and storage matrices, are listed in Table 2.

The output-oriented diact transaction types can be formulated in parallel as discussed in Appendix F. The output-oriented, flow-based diact flow and storage distribution matrices and the corresponding simple and composite flow and storage matrices are listed in Table 3. Their output-oriented, storage-based counterparts are also listed in the Supplementary Materials (Table S2).

It is worth noting that the input- and output-oriented diact flow and storage matrices represent the corresponding intercompartmental flow distributions and storage organizations in either orientations, and they are different. The flow-based diact flow and storage matrices and their storage-based counterparts are the same matrix measures formulated in terms of the cumulative flow and storage distribution matrices, $N$ and $S$, respectively. Ecologically, the determination of the diact flows and storages from compartmental storages through the storage-based distribution matrices may prove more practical, as experimentally quantifying storages is significantly easier than measuring system flows.

2.7. Quantitative definitions of interspecific interactions. Community ecology studies interaction types among species of ecological networks, such as food webs. In general, the interspecific interactions are structurally determined by the network topology without regard for system flows $[35,34]$. This determination, however, gets more complicated, if possible at all, with the increasing complexity of intricate food webs $[26,55]$. Moreover, multiple food chains of possibly different lengths between two species disallow the classification based on the length of the chains between two species [7]. A parametric classification is attempted through a utility analysis in the literature, but it has disadvantages due to the geometric series expansion used in the formulation of indirect contributions, as discussed in Section 2.4.1 [48, 40, 17, 15, 47]. Moreover, unlike some of these authors' claims, some interactions, such as amensalism, cannot be classified through the flow analysis, as explained below.

We propose a quantitative characterization and classification technique for the interspecific interactions within food webs using the diact flows in this section. As it uses system flows for characterization of interspecific interactions, this quantitative technique sets up a bridge between two main branches of ecology: ecosystem ecology and community ecology. Since the diact transactions quantify nutrient and energy 
transfers within food webs, nontrophic interactions that are not part of a food web cannot be categorized through the proposed technique. For example, amensalism is defined as an interaction where an organism inflicts harm to another organism without any effect received by itself, such as some common mould species producing antibiotics as a waste product that kill bacteria in their microenvironments. Since there is no nutrient or energy transfer involved in this process, such nontrophic interactions need to be determined and classified topologically or qualitatively.

The proposed methodology decomposes ecosystem flows and storages to the utmost level. The nutrient and energy flows from one species, directly or indirectly, to any other species within a food web are precisely determined. Therefore, the flow transfers between two species can be grouped into the natural binary categories of net positive transfer or no transfer. We will use these categories to quantitatively define the corresponding binary classification of interspecific interactions: neutralism and antagonism. Neutralism will be described as the theoretical, base-state where two species do not affect each other. Antagonism can then be defined as the interaction of two species that is beneficial for one partner, "predator," but detrimental for the other one, "prey." Being beneficial and detrimental in this description will quantitatively be defined as net positive and negative diact flow transmission from one species to another. Depending on the diact transaction type, the corresponding diact interspecific interactions will be categorized as directly, indirectly, acyclically, cyclically, and totally neutral or antagonistic interactions.

The sign and strength analyses of interspecific interactions will be introduced below. The sign analysis determines the neutral and antagonistic nature of the interactions - whether the interaction is beneficial or harmful to the species involved. Since the proposed methodology can quantitatively determine the net benefit received by the involved species from each other, the strength analysis quantifies the strength of these interactions. The sign and strength of the direct interaction between species $i$ and $j$ will be defined as

$$
\delta_{i j}^{\mathrm{d}}=\operatorname{sgn}\left(\tau_{i j}^{\mathrm{d}}-\tau_{j i}^{\mathrm{d}}\right) \quad \text { and } \quad \mu_{i j}^{\mathrm{d}}=\frac{\left|\tau_{i j}^{\mathrm{d}}-\tau_{j i}^{\mathrm{d}}\right|}{\tau_{i j}^{\mathrm{d}}+\tau_{j i}^{\mathrm{d}}}
$$

respectively, where $\operatorname{sgn}(\cdot)$ is the sign function. In community ecology, the positive and negative effects of one species on another are represented by $(+)$ and $(-)$ signs. Following the convention, instead of $(+1)$ and $(-1),(+)$ and $(-)$ notations will be used for the sign of the diact interactions. The strength, $\mu_{i j}^{\mathrm{d}}$, is defined to be zero, if both terms in its denominator are zero. Note that $0 \leq \mu_{i j}^{\mathrm{d}} \leq 1$.

For the analysis of diact interactions ranging from the individual and local to the system-wide and global scale, the strength of the direct interactions, $\mu_{i j}^{\mathrm{d}}$, can be formulated with the normalization by $\tau_{i j}^{\mathrm{d}}+\tau_{j i}^{\mathrm{d}}$, as in Eq. $2.69, \tau_{i j}^{\mathrm{t}}+\tau_{j i}^{\mathrm{t}}, \tau_{i}+\tau_{j}$, or $\sigma^{\tau}=\mathbf{1}^{T} \tau$ in the given order. As an example, for the analysis of local direct interactions at the global scale, its strength can be defined as $\mu_{i j}^{\mathrm{d}}=\left|\tau_{i j}^{\mathrm{d}}-\tau_{j i}^{\mathrm{d}}\right| / \sigma^{\tau}$.

The direct neutral relationship between species $i$ and $j$ and "predation" of species $i$ on $j$ can quantitatively be characterized, respectively, as follows:

$$
\tau_{i j}^{\mathrm{d}}-\tau_{j i}^{\mathrm{d}}=0 \Rightarrow \delta_{i j}^{\mathrm{d}}=(0) \quad \text { and } \quad \tau_{i j}^{\mathrm{d}}-\tau_{j i}^{\mathrm{d}}>0 \Rightarrow \delta_{i j}^{\mathrm{d}}=(+) .
$$

This classification can also be extended to the interactions between two species along a given food chain using the transient inflows. We will use the notations $\delta_{i j}^{w}$ and $\mu_{i j}^{w}$ for the sign and strength of the net flow from compartment $j$ indirectly to $i$ through trophic interactions along a given food chain, $p_{i j}^{w}$. The sign and strength of the 
other diact interactions, as well as their characterization can be formulated similarly by using the corresponding diact flows in Eqs. 2.69 and 2.70 instead of the direct flows. The standard interspecific interaction types, such as exploitation, mutualism, commensalism, and competition are also defined quantitatively through the proposed methodology in a separate article by [13].

We define ordered quintuples for the strength and sign of the diact interactions between species $i$ and $j$ as

$$
\mu_{i j}^{*}=\left(\mu_{i j}^{\mathrm{d}}, \mu_{i j}^{\mathrm{i}}, \mu_{i j}^{\mathrm{a}}, \mu_{i j}^{\mathrm{c}}, \mu_{i j}^{\mathrm{t}}\right) \quad \text { and } \quad \delta_{i j}^{*}=\left(\delta_{i j}^{\mathrm{d}}, \delta_{i j}^{\mathrm{i}}, \delta_{i j}^{\mathrm{a}}, \delta_{i j}^{\mathrm{c}}, \delta_{i j}^{\mathrm{t}}\right) .
$$

The elements of the ordered quintuples $\delta_{i j}^{*}$ and $\mu_{i j}^{*}$ represent the neutral and antagonistic nature of the diact interactions and their strength, respectively. As an example, $\delta_{i j}^{*}=(0,+,-,+,+)$ indicates that the interactions between $i$ and $j$ are directly neutral and indirectly, acyclically, cyclically, and totally antagonistic. Moreover, the antagonistic interactions are indirectly, cyclically and totally beneficial but acyclically detrimental to species $i$.

The storage-based quantitative definition of interspecific interactions can be formulated in parallel by substituting the corresponding diact storages instead of the diact flows in the definitions above. For classification of the interspecific interactions induced by individual environmental inputs, the simple diact flows and storages can be used instead of their composite counterparts in Eqs. 2.70, 2.69, and 2.71. Applications of the proposed quantitative classification are presented in the case studies.

2.8. System measures and indices. The system partitioning methodology yields the subthroughflow and substorage matrices that measure environmental influence on system compartments in terms of the flow and storage generation. These matrix measures enable the determination of the fate of environmental inputs individually and separately within the system. For the quantification of intercompartmental flow and storage dynamics, the subsystem partitioning methodology then formulates the transient and diact flows and storages. The transient and diact transactions enable tracking arbitrary intercompartmental flows and storages along all or particular flow paths within the system and determining the influence of system compartments directly and indirectly on each other. The output-oriented system analysis develops the corresponding measures and has the same functionalities based on environmental outputs instead of inputs.

Many other dynamic and static system analysis tools, such as measures and indices for the diact effects, utilities, exposures, and residence times, as well as the system efficiencies, stress, and resilience can be formulated based on the proposed methodology. The dynamic measures and indices of matrix, vector, and scalar types have recently been introduced as quantitative ecosystem indicators by [11]. The static versions of these mathematical system analysis tools have also been formulated in a separate work [13].

3. Results. The proposed methodology is applied to two ecological network models from the literature in this section. The numerical results for the ecosystem measures developed in the present paper, such as the substorage and subthroughflow matrices, as well as the transient and diact flows and storages, are presented. Additional case studies are also provided in the Supplementary Materials (Section S5) for the presentation of various other aspects of the proposed methodology.

The results indicate that the proposed methodology precisely quantifies system characteristics, functions, and behaviors, tracks the fate of environmental inputs and 


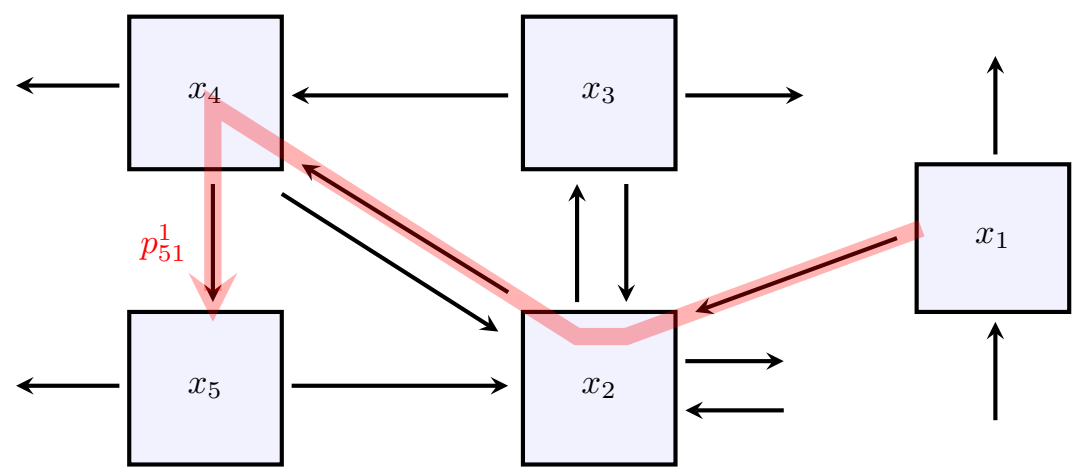

FIG. 5. Schematic representation of the model network. Flow path $p_{51}^{1}$ along which the transient subflows and substorages are computed is red (subsystems are not shown) (Case Study 3.1).

intercompartmental flows as well as associated storages within the system, and, thus, can be used for rigorous analysis of ecosystems. Although the models are extensively studied in the literature, some of these results have not yet been reported. It is worth emphasizing, however, that this present work proposes a mathematical method - a systematic technique designed for analyzing ecological network models at steady state - and it is not itself a model. Therefore, we focus more on demonstrating the efficiency and wide applicability of the method. It is expected that once the method is accessible to a broader community of environmental ecologists, it can be used for ecological inferences and detailed analyses of specific models of interest.

3.1. Case Study. The Cone Spring ecosystem model is a commonly studied ecological network, first proposed by [46]. The analysis of the model through the proposed methodology is presented in this case study.

Cone Spring is a small, shallow spring-brook located in Louisa County, Iowa. The study area consists of $116 \mathrm{~m}^{2}$. The network has 5 compartments: 1 -plants, 2-detritus, 3-bacteria, 4-detritus feeders, and 5-carnivores. These compartments are connected by the transaction of energy between them. Therefore, the conserved quantity in question is energy for this ecosystem model. The system flow regime is given as follows:

$$
F=\left[\begin{array}{ccccc}
0 & 0 & 0 & 0 & 0 \\
8881 & 0 & 1600 & 200 & 167 \\
0 & 5205 & 0 & 0 & 0 \\
0 & 2309 & 75 & 0 & 0 \\
0 & 0 & 0 & 370 & 0
\end{array}\right], \quad y=\left[\begin{array}{c}
2303 \\
3969 \\
3530 \\
1814 \\
203
\end{array}\right], \quad z=\left[\begin{array}{c}
11184 \\
635 \\
0 \\
0 \\
0
\end{array}\right]
$$

The storages values are $x=[285,3579.4,116.6,60,17]^{T}[41]$. The units of energy flows and storages are $\left(\mathrm{kkal} \mathrm{m}^{-2} \mathrm{y}^{-1}\right)$ and $\left(\mathrm{kkal} \mathrm{m}^{-2}\right)$, respectively.

The system partitioning methodology produces the subthroguhflow matrix as formulated in Eq. 2.15:

$$
T=\left[\begin{array}{ccccc}
11184 & 0 & 0 & 0 & 0 \\
10717 & 766 & 0 & 0 & 0 \\
4858 & 347 & 0 & 0 & 0 \\
2225 & 159 & 0 & 0 & 0 \\
345 & 25 & 0 & 0 & 0
\end{array}\right]
$$


Since there is no environmental input into $3^{\text {th }}, 4^{\text {th }}$, and $5^{\text {th }}$ compartments $\left(z_{3}=z_{4}=\right.$ $\left.z_{5}=0\right)$, the $3^{\text {th }}, 4^{\text {th }}$, and $5^{\text {th }}$ subsystems represented on the corresponding columns of $T$ (and of $X$ ) are null. That is, there is no subthroughflow at (and, therefore, no substorage in) the corresponding subcompartments of these subsystems.

The subsystem partitioning methodology yields the diact energy flows and storages as listed in Table 1. The composite direct flow matrix is $T^{\mathrm{d}}=F$. The composite indirect flow and storage distribution matrices are

$$
N^{\mathrm{i}}=\left[\begin{array}{ccccc}
0 & 0 & 0 & 0 & 0 \\
0.1641 & 0.1713 & 0.0121 & 0.0950 & 0.0839 \\
0.4343 & 0 & 0.1448 & 0.0811 & 0.2426 \\
0.1989 & 0.0065 & 0.0643 & 0.0371 & 0.1111 \\
0.0309 & 0.0323 & 0.0122 & 0 & 0.0173
\end{array}\right] \text { and } S^{\mathrm{i}}=\mathcal{R} N^{\mathrm{i}}
$$

where the residence time matrix is $\mathcal{R}=\operatorname{diag}([0.0255,0.3117,0.0224,0.0252,0.0460])$. Therefore, the composite indirect flow matrix, as formulated in Eq. 2.44, becomes

$$
T^{\mathrm{i}}=N^{\mathrm{i}} \mathcal{T}=\left[\begin{array}{ccccc}
0 & 0 & 0 & 0 & 0 \\
1835.74 & 1967.00 & 63.02 & 226.41 & 31.04 \\
4857.67 & 0 & 753.81 & 193.28 & 89.76 \\
2224.91 & 75.00 & 334.40 & 88.52 & 41.11 \\
345.31 & 370.00 & 63.53 & 0 & 6.38
\end{array}\right]
$$

where $\mathcal{T}=\operatorname{diag}([11184,11483,5205,2384,370])$. Note that $\tau_{32}^{\mathrm{i}}=\tau_{54}^{\mathrm{i}}=0$. This is because of the fact that, although there are direct energy flows from the detritus (compartment 2) to the bacteria (3), $f_{32}=5205$, and from the detritus feeders (4) to the carnivores (5), $f_{54}=370$, there is no indirect flow in the same directions (see Fig. 5). There is no indirect energy flow to the plants (compartment 1) from any other compartments either, so the first row vector of $T^{\mathrm{i}}$ is zero. The associated composite indirect energy storages generated by these indirect flows are

$$
X^{\mathbf{i}}=\left[\begin{array}{ccccc}
0 & 0 & 0 & 0 & 0 \\
572.22 & 613.14 & 19.65 & 70.58 & 9.68 \\
108.81 & 0 & 16.89 & 4.32 & 2.01 \\
56.0 & 1.89 & 8.42 & 2.23 & 1.03 \\
15.87 & 17.0 & 2.91 & 0 & 0.29
\end{array}\right] .
$$

The diagonal entries of $T^{\mathrm{i}}$ and $X^{\mathrm{i}}$ are the composite cycling flows and associated storages, respectively. Those for the carnivores are $\tau_{55}^{\mathrm{c}}=\tau_{55}^{\mathrm{i}}=6.38 \mathrm{kkal} \mathrm{m}^{-2} \mathrm{y}^{-1}$ and $x_{55}^{\mathrm{c}}=x_{55}^{\mathrm{i}}=0.29 \mathrm{kkal} \mathrm{m}^{-2}$. That is, the given amounts of flow and storage are transmitted from the carnivores, indirectly through other compartments within the system, back into themselves.

The composite acyclic energy flow and storage matrices, $T^{\mathrm{a}}$ and $X^{\mathrm{a}}$, can also be computed as given in Table 1 . It can be shown that, $\tau_{54}^{\mathrm{a}} / \tau_{4}=0.152$ and $x_{54}^{\mathrm{a}} / x_{4}=$ 0.278 . In other words, the energy flow and storage transmitted from the detritus feeders to the carnivores that do not cycle after being transmitted are $15.2 \%$ and $27.8 \%$ of the outward energy throughflow at and storage in the detritus feeders, respectively. Since $27.8 \%$ of the energy storage in the detritus feeders is transferred to the carnivores, the ten percent energy transfer law from one trophic level to the next does not seem to be an accurate estimate for this food web [31]. As this and the similar results for the Neuse River Estuary ecosystem analyzed in the next case study illustrate, the 
proposed methodology constructs a quantitative base for testing empirical ecological hypotheses and, potentially, theoretical developments.

The composite transfer flow matrix $T^{\mathrm{t}}$, as formulated in Eq. 2.52, for the Cone Spring ecosystem then becomes

$$
T^{\mathrm{t}}=\left[\begin{array}{ccccc}
0 & 0 & 0 & 0 & 0 \\
10716.74 & 1967.00 & 1663.02 & 426.42 & 198.04 \\
4857.67 & 5205.00 & 753.81 & 193.28 & 89.76 \\
2224.91 & 2384.00 & 409.40 & 88.53 & 41.12 \\
345.31 & 370.00 & 63.54 & 370.00 & 6.38
\end{array}\right]
$$

This model is studied by [45] for similar purposes. The authors formulated a matrix measure called the total flow matrix in their work. Although it has a different derivation rationale, this total flow matrix is equivalent to the proposed input-oriented, flow-based, compartmental, composite transfer flow matrix given in Eq. 3.3.

The composite direct, indirect, and transfer flow matrices for the Cone Spring ecosystem model are presented above. It can be seen that $\tau_{52}^{\mathrm{d}}=f_{52}=0<167=\tau_{25}^{\mathrm{d}}=$ $f_{25}$, and, therefore, $\tau_{52}^{\mathrm{d}}-\tau_{25}^{\mathrm{d}}<0$. This result indicates that there is a net energy flow from the carnivores to the detritus through direct transactions. Similar computations show that $\tau_{52}^{\mathrm{i}}-\tau_{25}^{\mathrm{i}}=338.96>0, \tau_{52}^{\mathrm{a}}-\tau_{25}^{\mathrm{a}}=199.50>0, \tau_{52}^{\mathrm{c}}-\tau_{25}^{\mathrm{c}}=-27.54<0$, and $\tau_{52}^{\mathrm{t}}-\tau_{25}^{\mathrm{t}}=171.96>0$. Therefore, the ordered quintuples defined for quantitative classification of diact interactions and their strength in Section 2.7 become $\delta_{52}^{*}=$ $(-,+,+,-,+)$ and $\mu_{52}^{*}=(1.00,0.85,0.38,0.68,0.30)$ (see Fig. 5$)$. Consequently, the interactions between the carnivores and detritus are antagonistic with respect to all diact transaction types. More specifically, the carnivores receives net benefit from the detritus through indirect and acyclic interactions, but the detritus does so from the carnivores through direct and cycling interactions. Since the indirect and acyclic interactions dominate their relationship, the carnivores receive net benefit from their overall interactions. The carnivores and detritus are more antagonistic in their direct interactions $\left(\mu_{52}^{\mathrm{d}}=1.00\right)$ and less so in their overall interactions $\left(\mu_{52}^{\mathrm{t}}=0.30\right)$. That is, while the relative direct effect of these compartments on each other is large, their relative total effect on each other is smaller. Considering the influence of only two corresponding inputs, $z_{5}$ and $z_{2}$, because $z_{5}=0$, similar computations with the simple diact flows indicate that the nature of their diact interactions changes to $\tilde{\delta}_{52}^{*}=(0,+,+,+,+)$ with the corresponding strengths of $\tilde{\mu}_{52}^{*}=(0,1,1,1,1)$.

Similar analysis shows that the interspecific interactions between the carnivores and detritus feeders can be characterized as $\left(\delta_{54}^{\mathrm{d}}, \delta_{54}^{\mathrm{i}}, \delta_{54}^{\mathrm{a}}, \delta_{54}^{\mathrm{c}}, \delta_{54}^{\mathrm{t}}\right)=(+,-,+,+,+)$. Interestingly, the result indicates that, while the carnivores directly prey on the detritus feeders, the latter indirectly "feed" on the former. In this case, the direct transactions dominates total interactions between these species. Lastly, the interaction between the carnivores and bacteria can be identified as $\left(\delta_{53}^{\mathrm{d}}, \delta_{53}^{\mathrm{i}}, \delta_{53}^{\mathrm{a}}, \delta_{53}^{\mathrm{c}}, \delta_{53}^{\mathrm{t}}\right)=$ $(0,-,-,-,-)$. This implies that the carnivores and the bacteria are directly neutral species. On the other hand, the bacteria receives net benefit from the carnivores with respect to all the other diact transaction types, and this characterize their overall interactions.

The simple cycling and acyclic flow matrices for the Cone Spring ecosystem model 
are also given below:

$$
\tilde{T}^{\mathrm{c}}=\left[\begin{array}{ccccc}
0 & 0 & 0 & 0 & 0 \\
1835.74 & 131.26 & 0 & 0 & 0 \\
703.51 & 50.30 & 0 & 0 & 0 \\
82.62 & 5.91 & 0 & 0 & 0 \\
5.96 & 0.43 & 0 & 0 & 0
\end{array}\right] \text { and } \quad \tilde{T}^{\mathrm{a}}=\left[\begin{array}{ccccc}
0 & 0 & 0 & 0 & 0 \\
8881 & 0 & 0 & 0 & 0 \\
4154.16 & 297.03 & 0 & 0 & 0 \\
2142.30 & 153.18 & 0 & 0 & 0 \\
339.35 & 24.26 & 0 & 0 & 0
\end{array}\right]
$$

It can be shown that $\tilde{\mathcal{T}}^{\mathrm{c}}=\operatorname{diag}\left(\tilde{T}^{\mathrm{c}} \mathbf{1}\right)=\operatorname{diag}\left(T^{\mathrm{c}}\right)=\operatorname{diag}\left(T^{\mathrm{t}}\right)=\operatorname{diag}\left(T^{\mathrm{i}}\right)$ as formulated in Eq. 2.66. A comparison of the corresponding nondiagonal entries of $\tilde{T}^{c}$ and $\tilde{T}^{\mathrm{a}}$ also shows that the simple acyclic flows are about an order of magnitude greater than the simple cycling flows. This observation indicates that, unlike the Neuse River Estuary ecosystem analyzed in the next case study, the simple acyclic flows dominates the cycling flows in the Cone Spring ecosystem. The dominance of different diact transactions ascertains one of the identifying characteristics of these two ecosystems [13].

For a given arbitrary environmental energy input $\mathcal{Z}^{\prime}=\operatorname{diag}([1,2,3,4,5])$, the energy storage organizations, $X^{[1]}, X^{[2]}$, and $X^{[3]}$, along all possible food chains of length 1,2 , and 3 , as well as the final energy storage organization, $X$, can be computed using Eq. D.3:

$$
\begin{aligned}
X^{[1]} & =\left[\begin{array}{ccccc}
0.025 & 0 & 0 & 0 & 0 \\
0.248 & 0.623 & 0.287 & 0.105 & 0.703 \\
0 & 0.020 & 0.067 & 0 & 0 \\
0 & 0.010 & 0.001 & 0.101 & 0 \\
0 & 0 & 0 & 0.029 & 0.230
\end{array}\right], \\
X^{[2]} & =\left[\begin{array}{ccccc}
0.025 & 0 & 0 & 0 & 0 \\
0.248 & 0.721 & 0.289 & 0.192 & 0.703 \\
0.008 & 0.020 & 0.077 & 0.003 & 0.023 \\
0.004 & 0.010 & 0.006 & 0.102 & 0.011 \\
0 & 0.003 & 0.000 & 0.029 & 0.230
\end{array}\right], \\
X^{[3]} & =\left[\begin{array}{ccccc}
0.025 & 0 & 0 & 0 & 0 \\
0.286 & 0.730 & 0.334 & 0.208 & 0.813 \\
0.008 & 0.023 & 0.077 & 0.006 & 0.023 \\
0.004 & 0.012 & 0.006 & 0.104 & 0.012 \\
0.001 & 0.003 & 0.002 & 0.029 & 0.233
\end{array}\right],
\end{aligned}
$$

and

$$
X=\left[\begin{array}{ccccc}
0.025 & 0 & 0 & 0 & 0 \\
0.299 & 0.752 & 0.349 & 0.232 & 0.849 \\
0.010 & 0.025 & 0.079 & 0.008 & 0.028 \\
0.005 & 0.013 & 0.007 & 0.105 & 0.014 \\
0.001 & 0.004 & 0.002 & 0.030 & 0.234
\end{array}\right]
$$

The transient flows and storages generated by a single environmental nitrogen input at each step along a given specific flow path within the Neuse River Estuary ecosystem are presented in Eq. 3.4. The transient energy storage matrices for the Cone Spring ecosystem model presented above show that the subsystem partitioning also enables 
tracking the fate of environmental inputs within the entire system at each step along all possible food chains of arbitrary length.

The input- and output-oriented subsystem partitioning methodology can be used to determine the energy flows and storages generated and dissipated along a particular food chain, respectively. Considering the food chain $p_{51}^{1}=1 \mapsto 1 \rightsquigarrow 2 \rightsquigarrow 4 \rightarrow 5$ and its reverse $\bar{p}_{15}^{1}=0 \mapsto 5 \rightsquigarrow 4 \rightsquigarrow 2 \rightarrow 1$ (see Fig. 5), Eq. 2.31 yields

$$
\begin{array}{ll}
\check{\tau}_{5}^{1}=\tau_{1} q_{21}^{\tau} q_{42}^{\tau} q_{54}^{\tau}=277.16, & x_{5}^{1}=r_{5} \check{\tau}_{5}^{1}=12.73, \\
\check{\tau}_{1}^{1}=y_{5} \bar{q}_{45}^{\tau} \bar{q}_{24}^{\tau} \bar{q}_{12}^{\tau}=152.06, & \bar{x}_{1}^{1}=r_{1} \check{\tau}_{1}^{1}=3.87 .
\end{array}
$$

Here, $\check{\tau}_{5}^{1}$ and $\check{\tau}_{1}^{1}$ are the input- and output-oriented transient subflows generated by the corresponding local inputs $\tau_{1}$ and $y_{5}$ at compartment 5 forward along the flow path, $p_{51}^{1}$, and at compartment 1 along its reverse, $\bar{p}_{15}^{1}$, respectively. The associated transient substorages $x_{5}^{1}$ and $\bar{x}_{1}^{1}$ are generated by the corresponding transient subflows. These results indicate that, $\check{\tau}_{5}^{1}=277.16 \mathrm{kkal} \mathrm{m}^{-2} \mathrm{y}^{-1}$ of the throughflow at the plants, $\tau_{1}=11184 \mathrm{kkal} \mathrm{m}^{-2} \mathrm{y}^{-1}$, is transmitted to the carnivores along the given food chain. This transient throughflow generates $x_{5}^{1}=12.73 \mathrm{kkal} \mathrm{m}^{-2}$ of the total energy stored in the carnivores, $x_{5}=17 \mathrm{kkal} \mathrm{m}^{-2}$. Since the input-oriented transient subflows at the terminal compartment and the output-oriented transient subflows at the initial compartment along a linear flow path are the same, as shown in Appendix G, this transient subflow, $\check{\tau}_{5}^{1}$, emanates from $r_{1} \check{\tau}_{5}^{1}=7.06 \mathrm{kkal} \mathrm{m}^{-2}$ of the total energy stored in the plants, $x_{1}=285 \mathrm{kkal} \mathrm{m}^{-2}$. Moreover, $\check{\bar{\tau}}_{1}^{1}=152.06 \mathrm{kkal} \mathrm{m}^{-2} \mathrm{y}^{-1}$ of the energy transmitted from the plants to the carnivores along the path is destined to dissipate after its transmission. That corresponds with $\bar{x}_{1}^{1}=3.87 \mathrm{kkal} \mathrm{m}^{-2}$ of the energy storage in the plants and $r_{5} \check{\tau}_{1}^{1}=6.99 \mathrm{kkal} \mathrm{m}^{-2}$ of the energy storage in the carnivores. Consequently, $7.06 / 285=2.48 \%$ of the energy storage in the plants is transferred to the carnivores along the path, which constitutes $12.73 / 17=74.9 \%$ of the energy storage in the carnivores. Also, $6.99 / 12.73=54.9 \%$ of this transferred energy is used up by the carnivores to carry out their life processes and dissipated as heat.

For output-oriented analysis of the Cone Spring ecosystem, the output-oriented substorage matrix $\bar{X}$ can be computed as formulated in Eq. F.6:

$$
\bar{X}=\left[\begin{array}{ccccc}
58.69 & 94.39 & 83.95 & 43.14 & 4.83 \\
0 & 1492.92 & 1327.79 & 682.33 & 76.36 \\
0 & 15.06 & 92.47 & 8.16 & 0.913 \\
0 & 3.86 & 3.43 & 47.42 & 5.31 \\
0 & 3.20 & 2.85 & 1.46 & 9.49
\end{array}\right]
$$

The output-oriented subthroughflow matrix, $\bar{T}$, can be computed similarly using Eq. F.7 as well. All the entries on the first column of $\bar{X}$ are zero except $\bar{x}_{1_{1}}$. This indicates that the energy storage in the plants destined to exit the system as output is $\bar{x}_{1_{1}}=58.69 \mathrm{kkal} \mathrm{m}^{-2}$, and no other species has energy storage that ends up with the same fate. The entries on the last row show that the minimum energy storage in the carnivores destined to exit the system as output is via the detritus feeders, $\bar{x}_{5_{4}}=1.46$ kkal m${ }^{-2}$. That is, this amount of energy stored in the carnivores is transmitted to and used up by the detritus feeders for their life processes and dissipated as heat.

The output-oriented diact flows and storages are formulated in Appendix F. Among them, the simple acyclic storage matrix, $\tilde{\bar{X}}^{a}$, for the Cone Spring ecosystem 


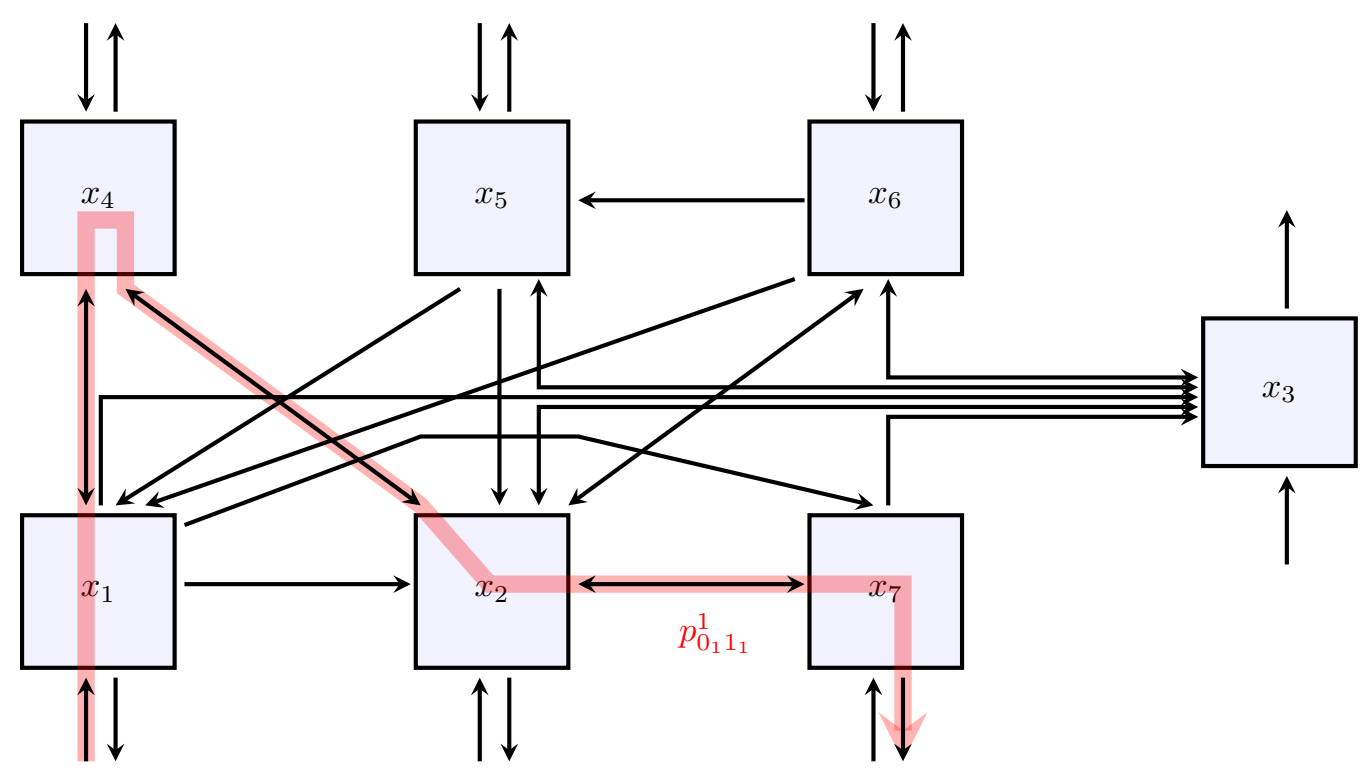

FIG. 6. Schematic representation of the model network. Subflow path $p_{0_{1} 1_{1}}^{1}$ along which the transient subflows and substorages are computed is red (subsystems are not shown) (Case Study 3.2).

becomes

$$
\tilde{\bar{X}}^{\mathrm{a}}=\left[\begin{array}{ccccc}
0 & 94.39 & 83.95 & 43.14 & 4.83 \\
0 & 0 & 1100.35 & 565.45 & 63.28 \\
0 & 12.88 & 0 & 6.98 & 0.78 \\
0 & 3.71 & 3.30 & 0 & 5.11 \\
0 & 3.15 & 2.80 & 1.44 & 0
\end{array}\right]
$$

As seen from the results, the corresponding nondiagonal entries of $\bar{X}$ and $\tilde{\bar{X}}^{a}$ are either equal or close to each other. The difference of $\bar{x}_{5_{4}}^{\mathrm{c}}=\bar{x}_{5_{4}}-\bar{x}_{5_{4}}^{\mathrm{a}}=1.4627-1.4374=$ $0.025 \mathrm{kkal} \mathrm{m}^{-2}$, for example, indicates that only a small amount of energy, $\bar{x}_{5_{4}}^{\mathrm{c}}$, completes its cycle in the carnivores before being transmitted to the detritus feeders and then dissipated as heat. This is another manifestation of insignificant cycling activity within the Cone Spring ecosystem. Such differences between the dominance of the diact transaction types can be used for the characterization and classification of ecosystems as discussed above and in the next case study.

All the other output-oriented subthroughflows, substorages, transient and diact flows and storages can also be computed similar to their input-oriented counterparts presented in the discussion above and interpreted accordingly for various aspects of the Cone Spring ecosystem.

3.2. Case Study. The Neuse River Estuary is a drowned river valley located at the transition from the Neuse River to Pamlico Sound in North Carolina. In 1997, the State of North Carolina legislated a reduction in nitrogen loading to the estuary. As part of the monitoring program to study the estuary's response to new environmental management, nitrogen loading data is constructed for 16 seasons starting from Spring 1985 to Winter 1989 [9].

The ecosystem is modeled with seven compartments: phytoplankton particulate 

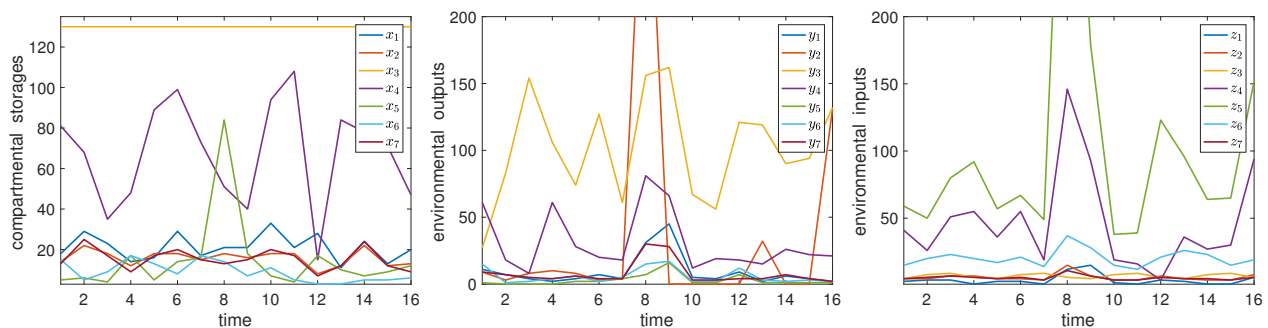

FIG. 7. The storage, $x_{i}$, output, $y_{i}$, and input, $z_{i}$, values at each time step. In the first graph, $x_{3}$ is scaled down by a factor of $10^{-1}$ for clarity of presentation (Case Study 3.2).

nitrogen, 1-PN-phyto; heterotroph particulate nitrogen, 2-PN-hetero; sediment particulate nitrogen, 3-N-sed; dissolved organic nitrogen, 4-DON; nitrate and nitrites, 5-NOx; ammonium, 6-NH4; and abiotic particulate nitrogen, 7-PN-abiotic. The compartments will be indexed in the given order; for example, $x_{1}$ represents the nitrogen storage in PN-phyto. The units for nitrogen storage and flow are $\left(\mathrm{mmol} \mathrm{m}^{-2}\right)$ and $\left(\mathrm{mmol} \mathrm{m} \mathrm{m}^{-2}\right.$ season $\left.^{-1}\right)$, respectively. The conserved quantity of interest in this case is nitrogen. Each season is considered to be a discrete time step; for example, $t=1$ corresponds to Spring 1985 and $t=16$ to Winter 1989. The system, with these seven compartments $(n=7), 22$ intercompartmental flows, environmental inputs, and outputs, is at steady state at each time step. The details of the rules and procedures for converting field data to flows and standing stocks needed for network construction are given by [9] (see Fig. 6).

We used a quasi-steady-state approach to analyze and interpret the data. That is, the system is solved at each data time step for each season under the steady-state assumption, and then the results are combined to get the impression of potential dynamic behavior. The system information - the storages and environmental inputs and outputs - is presented in Fig. 7 to give an overall impression of the system flows and storages.

The system partitioning methodology yields the subthrougflow and substorage matrix measures that determine the distribution of environmental inputs and the organization of associated storages within the system. The componentwise and timewise cross sections of the system are presented in Fig. 8. Timewise, the substorage and subthroughflow matrices at time $t=3$ (Fall 1985), $X(3)$ and $T(3)$, are presented. For a componentwise cross section of the system, the substorages in and subthroughflows at a specific compartment, $x_{1}$, are also presented for a typical compartmental behavior. Moreover, the substorage and subthroughflow vectors for the first subsystem, $\left[x_{1_{1}}, x_{2_{1}} \ldots, x_{7_{1}}\right]^{T}$ and $\left[\tau_{1_{1}}, \tau_{2_{1}} \ldots, \tau_{7_{1}}\right]^{T}$, which are derived from the environmental input into the first compartment (PN-phyto), $z_{1}$, are also depicted in Fig. 8. At first glance, comparing the system information in Fig. 7 and the system partitioning results in Fig. 8, it can be observed that seasonality - the almost periodic behavior per 4 time steps (a year) - is more pronounced in subsystem substorages and subthroughflows. This result indicates that, the system partitioning can better capture temporal behavior of ecological systems.

In Fig. 8, the figure for the entries of substorage matrix $X(3)$ shows the organization of the nitrogen storages generated by the environmental inputs within the system at time $t=3$. The results indicate that there is an excess amount of nitrogen in all subcompartments of compartment $3\left(\mathrm{~N}\right.$-sed) at $t=3$. This implies that, $x_{3}$ is the major recipient of nitrogen derived from all environmental inputs during Fall 

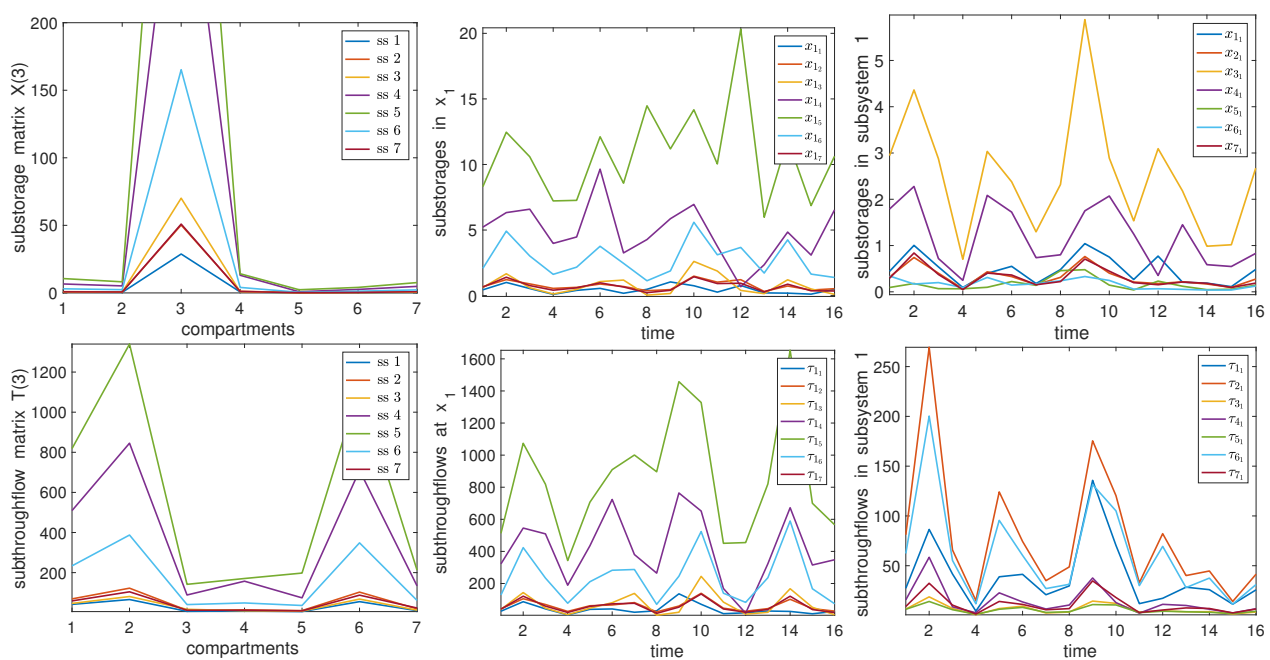

FIG. 8. The numerical results for selected elements of the substorage and subthroughflow matrix measures, $X$ and $T$. The legends (ss $1-7)$ in the first figures of each row are the subsystem numbers (column numbers of the matrices). In the matrix of $2 \times 3$ figure boxes, in the figure at $(1,3)-$ position (the substorages in subsystem 1), $x_{3_{1}}$ is scaled down by a factor of $10^{-1}$ for clarity of the presentation (Case Study 3.2).

1985. The largest nitrogen substorage, $x_{35}(3)$, is at subcompartment $3_{5}$ due to a large amount of nitrogen input into the system at compartment 5 (NOx), $z_{5}(3)$. The (scaled) storage $x_{3}$ and input $z_{5}$ are presented at each time step in Fig. 7.

The figure for the entries of subthroughflow matrix $T(3)$ in Fig. 8 illustrates the distribution of the environmental nitrogen inputs within the system at time $t=3$. The graphs show that the nitrogen subthroughflows generated by input $z_{5}(3)$ are larger at compartments 2 (PN-hetero) and $6(\mathrm{NH} 4)$. That is, $\tau_{2_{5}}(3)$ and $\tau_{6_{5}}(3)$ are the two largest subthroughflows generated by input $z_{5}(3)$. Another observation is that the graphs for the subthroughflows in subsystem 1 in Fig. 8 show almost a periodic behavior at every 4 time steps (a year), which is likely a manifestation of the seasonality. Similar interpretations of the system information at both compartmental or subcompartmental levels bring out detailed and precise information about the ecosystem function and behavior.

In general terms, $x_{i}$ is the composite nitrogen storage in compartment $i$ of the original system. It cannot be used to distinguish the nitrogen derived from individual environmental inputs. On the other hand, $x_{i_{k}}$ is the nitrogen storage in compartment $i$ that is generated by individual environmental input $z_{k}$. Therefore, the proposed system partitioning methodology allows for partitioning composite compartmental nitrogen into subcompartmental nitrogen segments based on their constituent environmental sources. In other words, the system partitioning enables tracking the fate of environmental nitrogen inputs individually and separately within the system. The proposed subsystem partitioning methodology then allows for partitioning an arbitrary composite intercompartmental nitrogen flow and the associated nitrogen storages generated by this flow into segments in each subcompartment along a given set of subflow paths. In other words, the subsystem partitioning enables tracking the fate of arbitrary intercompartmental nitrogen flows and associated storages within the subsystems. Consequently, the effect of one compartment on another in terms of 

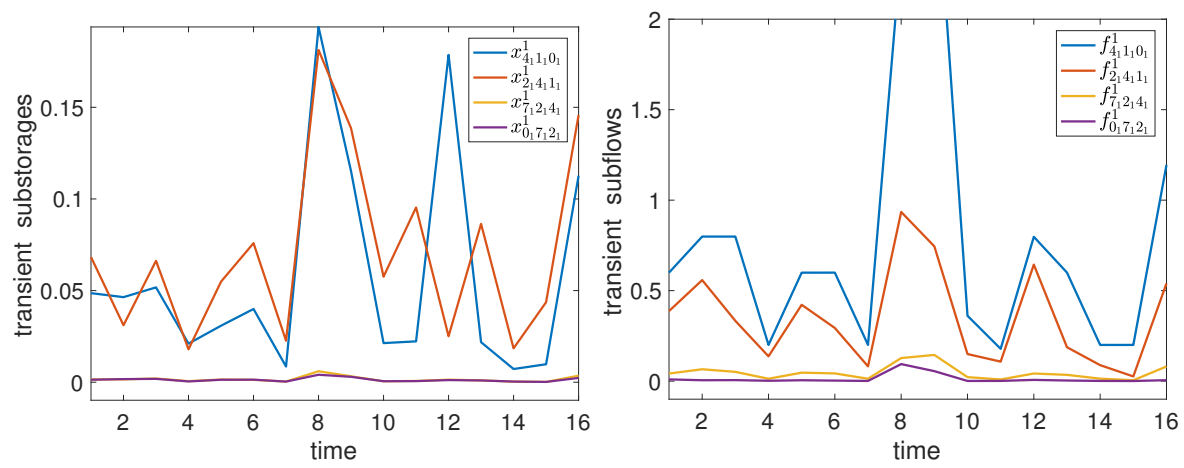

FIG. 9. Transient subflows and substorages along path $p_{0_{1} 1_{1}}^{1}$. The transient subflow $f_{0_{1} 7_{1} 2_{1}}^{1}$ is scaled up by a factor of 10 for clarity of the presentation (Case Study 3.2).

not only direct but also indirect nitrogen transactions can be determined. Parallel interpretations are also valid for the nitrogen throughflows of the original system, $\tau_{i}$, and the subthroughflows of the decomposed system, $\tau_{i_{k}}$. These interpretations can be extended further to the output-oriented system and subsystem partitioning as well. We will present some numerical results from the application of the subsystem partitioning methodology below.

The subsystem partitioning formulated in Eq. 2.27 will be used to compute the local output at subcompartment $7_{1}$ (the first subcompartment of PN-abiotic) generated by environmental input $z_{1}$ at subcompartment $1_{1}$ (the first subcompartment of PN-phyto) along subflow path $p_{0_{1} 1_{1}}^{1}$ defined as

$$
p_{0_{1} 1_{1}}^{1}=0_{1} \mapsto 1_{1} \rightsquigarrow 4_{1} \rightsquigarrow 2_{1} \rightsquigarrow 7_{1} \rightarrow 0_{1}
$$

(see Fig. 6). The transient subflows and substorages at each step along the path for $t=5$ are given below:

$$
\begin{aligned}
f_{4_{1} 1_{1} 0_{1}}^{1}(5)=0.60, & f_{2_{1} 4_{1} 1_{1}}^{1}(5)=0.42, \\
f_{7_{1} 2_{1} 4_{1}}^{1}(5)=0.05, & f_{0_{1} 7_{1} 2_{1}}^{1}(5)=0.05 \times 10^{-2}, \\
x_{4_{1} 1_{1} 0_{1}}^{1}(5)=0.03, & x_{2_{1} 4_{1} 1_{1}}^{1}(5)=0.05 \\
x_{7_{1} 2_{1} 4_{1}}^{1}(5)=0.15 \times 10^{-2}, & x_{0_{1} 7_{1} 2_{1}}^{1}(5)=0.13 \times 10^{-2} .
\end{aligned}
$$

Similar computations yield transient subflows and substorages at each time step, and these discrete functions are presented in Fig. 9. It can be shown that $f_{0_{1} 7_{1} 2_{1}}^{1}(t) \leq$ $0.94 \times 10^{-2} \mathrm{mmol} \mathrm{m}^{-2}$ season $^{-1}$ and, therefore, $f_{0_{1} 7_{1} 2_{1}}^{1}(t) / z_{1}(t) \leq 0.08 \times 10^{-2}$ for all time steps. These relationships imply that, at most only a small fraction, that is, $0.08 \%$ of the environmental input $z_{1}$ exits the system from subcompartment $7_{1}$ as output after traveling along subflow path $p_{0_{1} 1_{1}}^{1}$ within the system.

The subsystem partitioning is independent of system partitioning, as outlined in Section 2.4.1. Using a flow path starting from compartment 1 (PN-phyto) and ending at 7 (PN-abiotic) parallel to subflow path $p_{0_{1} 1_{1}}^{1}, p_{71}^{1}=1 \mapsto 1 \rightsquigarrow 4 \rightsquigarrow 2 \rightarrow 7$, the transient flows and storages can be computed similarly at $t=5$. Since this flow path starts at compartment 1 , the local input becomes $\tau_{1}$ (see the Supplementary Materials, Section S2.1). If the flow path is reversed, the transient flows and storages from compartment 7 to 1 can also be computed along the reversed path, $p_{17}^{1}=7 \mapsto$ 
$7 \rightsquigarrow 2 \rightsquigarrow 4 \rightarrow 1$, with the local inflow of $\tau_{7}$. The following results are obtained by using Eq. 2.31:

$$
f_{724}^{1}(5)=24.24 \quad \text { and } \quad f_{142}^{1}(5)=14.96 \Rightarrow \operatorname{sgn}\left(f_{724}^{1}(5)-f_{142}^{1}(5)\right)>0 .
$$

The difference between the transient inflows, $f_{724}^{1}(5)$ and $f_{142}^{1}(5)$ at compartments 7 and 1, respectively, determines the net indirect flow between these compartments along the given path. Therefore, the sign and strength of the interspecific interaction along the path defined in Section 2.7 becomes

$$
\delta_{71}^{1}(5)=(+) \quad \text { and } \quad \mu_{71}^{1}(5)=\frac{\left|f_{724}^{1}(5)-f_{142}^{1}(5)\right|}{f_{724}^{1}(5)+f_{142}^{1}(5)}=0.24
$$

In fact, it can be shown that $1.8<f_{724}^{1}(t)-f_{142}^{1}(t)<35.6$, and, therefore, $\delta_{71}^{1}(t)=(+)$ for all $t$ except $t=8$ and $t=13$ since $\delta_{71}^{1}(8)=\delta_{71}^{1}(13)=(-)$. At these two time steps, the direction of benefit between compartments 1 and 7 along the flow path is reversed. Consequently, most of the time, compartment 7 benefits from 1 indirectly along the given flow path. It can also be shown that the strength of this interaction oscillates almost periodically for every four seasons - mostly increasing during the summers and decreasing during the winters - and $0.03 \leq \mu_{71}^{1}(t) \leq 0.95$.

The ordered quintuples defined in Section 2.7 for the sign and strength of the diact interspecific interactions between compartments 7 and 1 at $t=5$ become $\delta_{71}^{*}(5)=(+,-,+,-,-)$ and $\mu_{71}^{*}(5)=(1.00,0.38,0.04,0.42,0.37)$. That is, compartments 1 and 7 are antagonistic with respect to all diact interactions. They are the most and least antagonistic directly and acyclically, respectively. The sign and stress analysis of this interaction can be done at each time step. The results indicate that compartment 7 receives net benefit from 1 directly and acyclically, but their interaction harms it indirectly and cyclically along all possible flow paths at any time $t$ (except $t=12$ ). The indirect and acyclic interactions dominates their overall interactions and, therefore, compartment 1 receives net total benefit from 7 .

Analyzing direct flows, even within a complex multi-compartmental system, is relatively straightforward. The direct, indirect, acyclic, cycling, and transfer (diact) flows and storages, however, are introduced systematically in the present paper. The composite indirect flow matrix at time $t=1$, for example, becomes

$$
T^{\mathbf{1}}(1)=\left[\begin{array}{ccccccc}
989.0 & 1053.4 & 743.3 & 774.0 & 744.8 & 327.6 & 803.3 \\
2564.6 & 3419.4 & 2266.6 & 2669.9 & 2818.7 & 1660.2 & 2399.0 \\
129.6 & 261.8 & 185.7 & 236.3 & 200.0 & 245.3 & 129.2 \\
416.9 & 210.5 & 460.4 & 588.2 & 556.3 & 640.9 & 501.1 \\
253.4 & 269.9 & 164.5 & 238.8 & 225.3 & 33.9 & 208.6 \\
2533.4 & 16.7 & 1882.2 & 2401.6 & 2244.4 & 2613.8 & 2069.9 \\
356.5 & 11.4 & 274.6 & 348.5 & 325.9 & 379.3 & 299.9
\end{array}\right]
$$

as formulated in Eq. 2.44. The $(i, k)$-element of $T^{\mathrm{i}}(1)$ represents the indirect flow from compartment $k$ to $i$ through other compartments within the system in Spring 1985. The indirect flow from compartment 2 (PN-hetero) to 7 (PN-abiotic), $T_{72}^{\mathrm{i}}=$ $11.4 \mathrm{mmol} \mathrm{m}^{-2}$ season $^{-1}$, seems to be the smallest of these interactions. The results indicate that each compartment has relatively large indirect flows to all the other compartments. A comparison of $T^{\mathrm{i}}(1)$ and $T^{\mathrm{d}}(1)=F(1)$ shows that the indirect interactions dominate the direct effects. The dominance of the diact flows and storages as an indicator for ecosystem characterization is reiterated and discussed further by [13]. 
The composite acyclic nitrogen flow and storage matrices, $T^{\mathrm{a}}$ and $X^{\mathrm{a}}$, can also be computed as given in Table 1 . It can be shown that

$$
\begin{aligned}
100 \frac{\tau_{21}^{\mathrm{a}}}{\tau_{1}} & =[11,5,9,20,7,7,5,35,10,3,8,20,10,4,8,21]^{T}, \\
100 \frac{x_{21}^{\mathrm{a}}}{x_{1}} & =[3,1,4,4,3,2,2,14,6,1,2,1,7,2,6,7]^{T} .
\end{aligned}
$$

The components of the vectors above represent the percentages at each time step of the inward nitrogen throughflow at and storage in compartment 2 (PN-hetero) transmitted from compartment 1 (PN-phyto) that do not cycle after their transmission. Since the nitrogen storage transfer percentages, $100 x_{21}^{\mathrm{a}}(t) / x_{1}(t)$, are significantly different from $10 \%$ at each time $t$, the ten percent energy transfer law from one trophic level to the next introduced by [31] does not seem to be an accurate estimate for this network either, similar to the Cone Spring ecosystem analyzed in the previous case study. Due to seasonality, these vectors show almost a periodic behavior in time, where the maximum acyclic transfer percentages are observed in the winters.

Considering the supplementary nature of the acyclic and cycling flows expressed in Eq. 2.60 we hypothesize that, possibly due to the slower biological activities during the winters, the transfer flows fall short of completing the nitrogen cycle and, therefore, the cycling flows decrease and the acyclic flows increase within the system during the winters. These results and interpretation are consistent with the periodic behaviors of the system acyclic flow and storage effect indices and corresponding acyclic efficiencies, as presented by $[11,13]$. These conclusions imply that such precise quantitative analysis of ecosystems may lead to more ecological theoretical developments.

The results presented above indicate that the proposed methodology brings out ecological inferences about the system characteristics, properties, and behaviors that are not available through the analysis of the original system by state-of-the-art techniques. New system measures and indices are also formulated based on the proposed methodology and used for further analysis of these ecosystems by [13].

4. Discussion. Environment is not an easy concept to define and analyze mathematically. Although sound rationales are offered in the literature for the analysis of natural system dynamics under special cases, such as linear systems and static models, realistically nature is always on the move and its systems are always changing to meet ever-renewing circumstances. The need for mathematically dynamic and nonlinear formulations has always been present. A mathematical methodology proposed recently for dynamic analysis of nonlinear compartmental systems by $[10,12]$ comprehensively addresses these shortcomings. The proposed mathematical methodology in the present paper is the static version of this dynamic methodology for the analysis of ecological networks at steady state.

Considering a hypothetical ecosystem modeling a food web with several interacting species for which the effect of a specific poison needs to be investigated, one of the most critical inquiries would be about the fate of the poison within the system. Current mathematical methods for static models can only analyze the composite throughflow of the toxin and the associated storage generated by this flow in each species. If multiple species are exposed to the same poison from the environment, however, the proposed system partitioning methodology enables partitioning the composite compartmental poison flow and storage in each species into subcompartmental segments based on their constituent environmental sources. In other words, the system partitioning enables tracking the fate of environmental poison inputs and 
associated storages individually and separately within the ecosystem. The proposed subsystem partitioning methodology can then track the fate of arbitrary intercompartmental poison flows and the associated storages generated by these flows in each species along given chains of interspecific interactions. Therefore, the spread of the arbitrary amount of toxin from one species to the entire system along all possible food chains can be monitored. Through the subsystem partitioning, the direct, indirect, acyclic, cycling, and transfer (diact) flows and storages of the poison from one compartment, directly or indirectly, to any other - including itself - can also be determined.

More technically, the mathematical method developed for the analysis of static ecological networks in the present article is based on the novel analytical and explicit system and subsystem partitioning methodologies. The system partitioning methodology explicitly generates mutually exclusive and exhaustive subsystems, each driven by a single environmental input, that are running within the original system and have the same structures and dynamics as the original system itself. The system partitioning yields the subthroughflow and substorage matrix measures that respectively represent the flows and storages generated by individual environmental inputs at each compartment separately. Equipped with these matrix measures, the system partitioning ascertain the distribution of environmental inputs and the organization of the associated storages generated by the inputs within the system. The system partitioning can be done based on both environmental inputs and outputs. Consequently, the composite compartmental storages and throughflows, $x_{i}$ and $\tau_{i}$, are partitioned into subcompartmental storage and throughflow segments, $x_{i_{k}}$ and $\tau_{i_{k}}\left(\bar{x}_{i_{k}}\right.$ and $\bar{\tau}_{i_{k}}$ for output-oriented analysis), based on their constituent environmental sources, $z_{k}\left(y_{k}\right)$. In brief, the system partitioning enables tracking the fate of environmental inputs (outputs) individually and separately within the system.

The subsystems are then further partitioned into subflows and associated substorages along a set of mutually exclusive and exhaustive directed subflow paths. The subsystem partitioning methodology yields the transient and diact flows and the associated storages generated by these flows. The transient subflows and associated substorages represent the distribution of arbitrary intercompartmental flows and the organization of the associated storages generated by these flows along given subflow paths within the subsystems. That is, arbitrary composite intercompartmental flows and storages can be partitioned into the constituent transient subflow and substorage segments along a given set of subflow paths. Consequently, the subsystem partitioning enables tracking the fate of arbitrary intercompartmental flows and storages within the subsystems. Therefore, the spread of an arbitrary flow or storage segment from one compartment to the entire system can be monitored. Moreover, a history of compartments visited by arbitrary system flows and storages can also be compiled. Based on the concept of transient flows and storages, the static direct, indirect, acyclic, cycling, and transfer (diact) flows and associated storages transmitted from one compartment, directly or indirectly, to any other within the system are systematically formulated for the quantification of intercompartmental flow and storage dynamics. The illustrative case studies in Section 3 and the Supplementary Materials (Section S5) demonstrate that these measures are rigorous and efficient ecological system analysis tools.

The proposed mathematical method, as a whole, decomposes the system flows and storages to the utmost level. An immediate consequence of this functionality is that the diact interactions between any two compartments of the system, in terms of flow and storage transfers, can be quantitatively determined. The precise mea- 
surements of the diact intercompartmental effects enable the analysis of interspecific interactions within food webs. A quantitative technique for the classification and characterization of the neutral and antagonistic nature of interspecific interactions and the determination of their strength is developed based on the diact flows and storages in the present manuscript.

The proposed method enables both input- and output-oriented analyses of static systems through the system partitioning methodology based on environmental inputs and outputs, respectively. The properties of input-oriented system and subsystem methodologies outlined above are similarly valid for their output-oriented counterparts. Briefly, while the intercompartmental flows and storages generated by environmental inputs are examined through the input-oriented analysis, those that are destined to exit the system as environmental outputs are investigated through the output-oriented analysis. The output-oriented system partitioning methodology enables partitioning the composite compartmental flows and storages into constituent subcompartmental segments destined to exit the system as environmental outputs separately from each compartment. The output-oriented subsystem partitioning then enables backtracking the fate of arbitrary storages and the associated inflows generating these storages at each compartment along a given flow path. Therefore, all possible contributing sources of a storage or associated inflow segment at a compartment can be backtracked within the entire system. Both input- and output-oriented analyses are formulated, and their duality is demonstrated through novel similarity relationships in the present paper.

For a comparison of the proposed methodology with state-of-the-art techniques, we first note that the proposed methodology refines the current static system analysis techniques from the compartmental level to the subcompartmental level, as outlined above. The analysis of direct transactions, even within a complex multicompartmental system, is relatively straightforward. On the other hand, the indirect flows and the associated storages generated by these flows from one compartment, indirectly through other compartments, to another have never been formulated before. Only the simple cycling and transfer (total) flows are introduced in the literature by $[19,20]$ and [45], respectively. The simple cycling and transfer storages generated by the corresponding flows are introduced in the present manuscript. In fact, the inputand output-oriented, flow- and storage-based, simple and composite diact flows and associated storages are also formulated systematically at both compartmental and subcompartmental levels in the present paper for the first time in literature.

This is the first comprehensive approach in literature that leads to a holistic methodology for ecosystem analysis. The existing static measures are reformulated with a different derivation rationale in the context of the proposed methodology and, therefore, are integrated with all the other novel system analysis tools introduced in the present work. Corrections in some current formulations are also suggested, as explained in Section 2.3 and 2.4. Moreover, through the proposed methodology, unique relationships among some current static measures are unveiled. The cumulative storage and throughflow distribution matrices, $S$ and $N$, have been treated separately in the literature; however, it is shown in Section 2.3 that they are related by a factor of the residence time matrix, $\mathcal{R}$ - a novel system measure introduced in Section 2.4. Also, the duality of input- and output-oriented system analyses establishes new similarity relationships between the cumulative storage and throughflow distribution matrices and integrates the system analyses in both orientations. A number of equivalent formulations for $S$ and $N$ are introduced in Section 2.3, too. The system and subsystem partitioning methodologies are also integrated through the geometric series expan- 
sions of the distribution matrices in Section 2.4. Consequently, various components of ecosystem analysis are effectively combined and holistically integrated within the proposed unifying mathematical framework. This holistic approach is reiterated and extended further by [13].

5. Conclusions. In the present paper, we developed a comprehensive mathematical method for the analysis of ecological systems at steady state. The proposed method is based on the novel analytical and explicit, mutually exclusive and exhaustive system and subsystem partitioning methodologies. While the proposed dynamic system partitioning yields the subthroughflow and substorage matrices for the distribution of environmental inputs and the organization of associated storages individually and separately within the system, the subsystem partitioning yields the transient and diact flow and storage matrices for the distribution of intercompartmental flows and the organization of associated storages. Consequently, the fate of environmental inputs and arbitrary intercompartmental flows, as well as the associated storages generated by these inputs and flows can be tracked individually and separately within the system. A quantitative technique for the characterization and classification of interspecific interactions and the determination of their strength within food webs is also developed based on the diact flows and storages.

Traditional ecology is still largely a descriptive empirical science. This narrows the field's scope of applicability and compromises its ability to deal with complex ecological networks. The proposed methodology extends the strength and applicability of state-of-the-art techniques and provides significant advancements in theory, methodology, and practicality. It serves as a quantitative platform for testing empirical hypotheses, ecological inferences, and, potentially, theoretical developments. Therefore, the method has the potential to lead the way to a more formalistic ecological science. We consider that the proposed methodology brings a novel complex system theory to the service of urgent and difficult environmental problems of the day. Several case studies from ecosystem ecology are presented to demonstrate the accuracy and efficiency of the proposed methodology.

The proposed methodology also constructs a base for the development of new mathematical system analysis tools as quantitative ecological indicators. Multiple such diact measures and indices of matrix, vector, and scalar type are systematically introduced in a separate paper by [13].

Appendices. Analytic solutions to linear systems, subsystem flows and storages, the static diact flows and storages, the output-oriented ecological network analysis, the duality of the input- and output-oriented analyses, and additional method formulations are presented in this section.

\section{Appendix A. Analytical solution to linear systems.}

In this section, we formulate analytical solutions to linear ecological systems with time dependent input as proposed by $[12,10]$.

The system partitioning methodology yields a linear system, if the original system is linear. That is, if Eq. 2.1 is linear, Eq. 2.3 takes the following linear form:

$$
\begin{aligned}
\dot{X}(t) & =\mathcal{Z}(t)+A(t) X(t), & X\left(t_{0}\right) & =\mathbf{0}, \\
\dot{x}_{0}(t) & =A(t) x_{0}(t), & & x_{0}\left(t_{0}\right)=x_{0} .
\end{aligned}
$$

Let $V(t)$ be the fundamental matrix solution to the system Eq. A.1 as formulated by 
[12]. That is, $V(t)$ is the unique solution of the system

$$
\dot{V}(t)=A(t) V(t), \quad V\left(t_{0}\right)=I .
$$

The solutions to Eq. A.1 for substorage matrix, $X(t)$, and initial substorage vector, $x_{0}(t)$, in terms of $V(t)$ become

$$
X(t)=\int_{t_{0}}^{t} V(t) V^{-1}(s) \mathcal{Z}(s) d s \quad \text { and } \quad x_{0}(t)=V(t) x_{0} .
$$

Therefore, the solution to the original system, Eq. 2.1, becomes

$$
x(t)=V(t) x_{0}+\int_{t_{0}}^{t} V(t) V^{-1}(s) z(s) d s .
$$

For the particular case of constant diagonalizable flow intensity matrix $A$, we have

$$
V(t)=\exp \left(\int_{t_{0}}^{t} A d s\right)=\mathrm{e}^{\left(t-t_{0}\right) A}=\Omega \mathrm{e}^{\left(t-t_{0}\right) \Lambda} \Omega^{-1}
$$

where $\Omega$ is the matrix whose columns are the eigenvectors of $A$, and $\Lambda$ is the diagonal matrix whose diagonal elements are the eigenvalues of $A$. For this particular case, Eq. A.2 takes the following form:

$$
X(t)=\int_{t_{0}}^{t} \mathrm{e}^{(t-s) A} \mathcal{Z}(s) d s \quad \text { and } \quad x_{0}(t)=\mathrm{e}^{\left(t-t_{0}\right) A} x_{0} .
$$

Consequently,

$$
x(t)=\mathrm{e}^{\left(t-t_{0}\right) A} x_{0}+\int_{t_{0}}^{t} \mathrm{e}^{(t-s) A} z(s) d s .
$$

A subsystem scaling argument is proposed to analyze system behavior per unit input in Section 2.3. The scaled substorage matrix, $S(t)=X(t) \mathcal{Z}^{-1}$, can be expressed for constant invertible input matrix, $\mathcal{Z}(t)=\mathcal{Z}>\mathbf{0}$, as follows:

$$
S(t)=\int_{t_{0}}^{t} V(t) V^{-1}(s) d s=\int_{t_{0}}^{t} \mathrm{e}^{(t-s) A} d s=\Omega\left(\int_{t_{0}}^{t} \mathrm{e}^{(t-s) \Lambda} d s\right) \Omega^{-1}
$$

using Eq. A.4.

The output-oriented counterpart of $S, \bar{S}$, will be discussed further below in Appendix F. An example of the analytic solution to a linear system, Eq. A.1, with time dependent environmental input is presented in the Supplementary Materials (Section S5.1).

\section{Appendix B. Subsystem flows and storages.}

The system partitioning methodology decomposes a system into mutually exclusive and exhaustive subsystems through a set of governing equations derived from subcompartmentalization and flow partitioning. This methodology enables the analysis of each subsystem generated by an environmental input individually and separately. The subsystem flows and storages in matrix form are formulated in this section.

The $k^{\text {th }}$ direct subflow matrix for the $k^{\text {th }}$ subsystem, $F_{k}=\left(f_{i_{k} j_{k}}\right)$, can be expressed as

$$
F_{k}=F \mathcal{X}^{-1} \mathcal{X}_{k}, \quad k=0, \ldots, n,
$$


where the $k^{\text {th }}$ substorage matrix $\mathcal{X}_{k}=\operatorname{diag}\left(\left[x_{1_{k}}, \ldots, x_{n_{k}}\right]\right)$ is the diagonal matrix of the substorage values in the $k^{t h}$ subsystem. The $k^{t h}$ input and output matrix functions are

$$
\mathcal{Y}_{k}=\mathcal{Y} \mathcal{X}^{-1} \mathcal{X}_{k} \quad \text { and } \quad \mathcal{Z}_{k}=\operatorname{diag}\left(z_{k} \boldsymbol{e}_{k}\right)
$$

respectively, where $\boldsymbol{e}_{k}$ is the elementary unit vector whose components are all zero except the $k^{t h}$ component, which is 1 , and $\boldsymbol{e}_{0}=\mathbf{0}$. The $k^{t h}$ direct subflow matrix, $F_{k}$, and the $k^{\text {th }}$ input and output vectors, $\check{z}_{k}=\mathcal{Z}_{k} \mathbf{1}$ and $\hat{y}_{k}=\mathcal{Y}_{k} \mathbf{1}$, are the counterparts for subsystem $k$ of the direct flow matrix, $F$, and input and output vectors, $z$ and $y$, for the original system. These matrices and vectors represent the subflow regime of the $k^{\text {th }}$ subsystem.

The solutions to the governing system, Eq. 2.9, for the substorage matrix, $X(t)$, and the initial substorage vector, $x_{0}(t)$, at steady state are given in Eq. 2.13. Using the notations and definitions of Eqs. 2.15 and B.1, the diagonal $k^{\text {th }}$ inward and outward subthroughflow matrices, $\check{\mathcal{T}}_{k}=\operatorname{diag}\left(\left[\check{\tau}_{1_{k}}, \ldots, \check{\tau}_{n_{k}}\right]\right)$ and $\hat{\mathcal{T}}_{k}=\operatorname{diag}\left(\left[\hat{\tau}_{1_{k}}, \ldots, \hat{\tau}_{n_{k}}\right]\right)$, for the $k^{t h}$ subsystem can be expressed as

$$
\begin{aligned}
& \check{\mathcal{T}}_{k}=\mathcal{Z}_{k}+\operatorname{diag}\left(F \mathcal{X}^{-1} \mathcal{X}_{k} \mathbf{1}\right), \\
& \hat{\mathcal{T}}_{k}=\left(\mathcal{Y}+\operatorname{diag}\left(F^{T} \mathbf{1}\right)\right) \mathcal{X}^{-1} \mathcal{X}_{k}=\mathcal{T} \mathcal{X}^{-1} \mathcal{X}_{k} .
\end{aligned}
$$

Due to the steady-state conditions, the $k^{\text {th }}$ inward and outward subthoroughflow matrices are equal, that is, $\mathcal{T}_{k}=\check{\mathcal{T}}_{k}=\hat{\mathcal{T}}_{k}$. The matrix $\mathcal{T}_{k}$ will be called the $k^{\text {th }}$ subthroughflow matrix. The steady-state conditions and Eq. B.2 imply that

$$
\check{\mathcal{T}}_{\ell} \check{\mathcal{T}}_{k}^{-1}=\hat{\mathcal{T}}_{\ell} \hat{\mathcal{T}}_{k}^{-1} \quad \text { and } \quad \mathcal{T}^{-1} \hat{\mathcal{T}}_{k}=\mathcal{X}^{-1} \mathcal{X}_{k}
$$

for any subsystem $k$ and $\ell$, provided that $\mathcal{T}_{k}$ is invertible [12]. If some diagonal entries of $\mathcal{T}_{k}$ are zero, these relationships are still valid componentwise for its nonzero diagonal elements, as given in Eq. E.2.

We define the decomposition and $k^{\text {th }}$ decomposition matrices, $D=\left(d_{i_{k}}\right)$ and $\mathcal{D}_{k}=\operatorname{diag}\left(\left[d_{1_{k}}, \ldots, d_{n_{k}}\right]\right)$, as

$$
D=\mathcal{X}^{-1} X=\mathcal{T}^{-1} T \text { and } \quad \mathcal{D}_{k}=\mathcal{X}^{-1} \mathcal{X}_{k}=\mathcal{T}^{-1} \mathcal{T}_{k} .
$$

The second equalities in the definitions of $D$ and $\mathcal{D}_{k}$ are due to Eqs. 2.15 and B.2. Using Eq. B.3, the $k^{\text {th }}$ direct subflow and substorage matrices, $F_{k}$ and $\mathcal{X}_{k}$, can then be written in various forms as follows:

$$
\mathcal{X}_{k}=\mathcal{R} \mathcal{T}_{k} \quad \text { and } \quad F_{k}=F \mathcal{D}_{k}=Q^{\tau} \mathcal{T}_{k}=Q^{x} \mathcal{X}_{k},
$$

similar to the relationships formulated for the decomposed system in Eq. 2.34.

The decomposition and $k^{t h}$ decomposition matrices, $D$ and $\mathcal{D}_{k}$, decompose the compartmental throughflow matrix, $\mathcal{T}$, into the subthroughflow and the $k^{\text {th }}$ subthroughflow matrices, $T$ and $\mathcal{T}_{k}$, as indicated in Eqs. 2.15 and B.2. That is,

$$
T=\mathcal{T} D \quad \text { and } \quad \mathcal{T}_{k}=\mathcal{T} \mathcal{D}_{k}
$$

The decomposition matrices can be considered as linear transformations as detailed in the next section.

The diagonal $k^{\text {th }}$ cumulative flow and storage distribution matrices can then be expressed as follows:

$$
\mathcal{N}_{k}=\mathcal{T}_{k} / z_{k} \quad \text { and } \quad \mathcal{S}_{k}=\mathcal{X}_{k} / z_{k} \quad \text { with } \quad \mathcal{S}_{k}=\mathcal{R} \mathcal{N}_{k} .
$$


The output-oriented subsystem flows and storages can be formulated using the substitutions defined in Eq. F.1 for the corresponding input-oriented counterparts.

\section{Appendix C. Matrix measures as linear transformations.}

The matrix measures introduced in the present manuscript can be considered as linear transformations. For example, Eqs. 2.19 and 2.20 indicate that the substorage and subthroughflow matrices, $S$ and $N$, are linear transformations that map environmental input vector to storage and throughflow vectors. That is,

$$
S: z \longrightarrow x \text { and } N: z \longrightarrow \tau .
$$

Similarly, we have

$$
S: \mathcal{Z} \longrightarrow X \quad \text { and } \quad N: \mathcal{Z} \longrightarrow T \text {. }
$$

The treatment of cumulative distribution matrices as linear transformations can be extended to the other decomposition and distribution matrices introduced in the present manuscript.

The decomposition matrices $D=\mathcal{X}^{-1} X$ and $\mathcal{D}_{k}=\mathcal{X}^{-1} \mathcal{X}_{k}$ can also be expressed as linear transformations that map the system flows and throughflows to the subflows and subthroughflows, as indicated in Eqs. 2.15, B.2, and B.5. That is,

$$
\mathcal{D}_{k}: F \longrightarrow F_{k}, \quad D: F \longrightarrow \tilde{T} \quad \text { and } \quad \mathcal{D}_{k}: \mathcal{T} \longrightarrow \mathcal{T}_{k}, \quad D: \mathcal{T} \longrightarrow T .
$$

Note that the decomposition matrices act by right multiplication on the system flow matrices.

The distribution matrices are also linear transformations that map transient storages and throughflows at step $(m), X^{(m)}$ and $T^{(m)}$, to transient storages or throughflows at the next step along all possible flow paths as formulated in Eqs. 2.33. That is,

$$
\begin{array}{rll}
\mathcal{R}: T^{(m)} \longrightarrow X^{(m)} & \text { and } & Q^{x}: X^{(m)} \longrightarrow T^{(m+1)} \\
L=\mathcal{R} Q^{x}: X^{(m)} \longrightarrow X^{(m+1)} & \text { and } & Q^{\tau}=Q^{x} \mathcal{R}: T^{(m)} \longrightarrow T^{(m+1)} .
\end{array}
$$

The direct storage distribution matrix $S^{\mathrm{d}}$ can also be considered as a linear transformation from $T^{(m)}$ to $X^{(m+1)}$ :

$$
S^{\mathrm{d}}: T^{(m)} \longrightarrow X^{(m+1)} .
$$

Moreover, the relationship $S^{\mathrm{d}}=\mathcal{R} Q^{\tau}=L \mathcal{R}$ indicates that $L$ and $Q^{\tau}$ are similar matrices with the same set of eigenvalues. If the distribution matrices are invertible, backtracking from the $(m+1)^{s t}$ to the $(m)^{\text {th }}$ step is possible using the relationships formulated in Eqs. 2.33. Note that we also have

$$
\mathcal{R}: T \longrightarrow X \text { and } \mathcal{R}: \tau \longrightarrow x
$$

due to the relationships $X=\mathcal{R} T$ and $x=\mathcal{R} \tau$ as demonstrated by [13].

The flow- and storage-based diact distribution matrices in both input- and output-orientations respectively map compartmental throughflows and storages to the corresponding diact flows and storages, as listed in Table 1. Therefore, the diact distribution matrices can be expressed as linear transformations. For input-oriented analysis,

$$
N^{*}: \mathcal{T} \longrightarrow T^{*} \text { and } \quad S^{*}: \mathcal{T} \longrightarrow X^{*}
$$


similar to Eq. C.1, where the superscript $\left(^{*}\right)$ in each relationship stands for any of the diact symbols. The storage-based, diact flow and storage distribution matrices can also be interpreted as linear transformations that map compartmental storages to diact flows and storages, as listed in Table 2. That is,

$$
N^{*, x}: \mathcal{X} \longrightarrow X^{*} \quad \text { and } \quad S^{*, x}: \mathcal{X} \longrightarrow T^{*}
$$

Appendix D. Geometric series expansion of matrix measures.

The invertibility of the substorage matrix $X$ is proved in the most general nonlinear dynamic setting by [12]. This implies that, $S$ and $N$ are also invertible due to Eqs. 2.23 and 2.26. Therefore, due to Eq. 2.22, $N$ can be expressed as the sum of the following infinite geometric series:

$$
N=\left(I-Q^{\tau}\right)^{-1}=I+Q^{\tau}+\left(Q^{\tau}\right)^{2}+\left(Q^{\tau}\right)^{3}+\cdots+\left(Q^{\tau}\right)^{m}+\cdots
$$

The term $\left(Q^{\tau}\right)^{m}$ represents the regular matrix multiplication of $Q^{\tau} m$ times by itself and, therefore, is the flow distribution matrix for the $m^{t h}$ step, and $\left(Q^{\tau}\right)^{0}=I$.

Starting with environmental input, the flows generated at each step add up to the subthroughflow matrix, $T$, as formulated in Eq. 2.21. That is,

$$
T=N \mathcal{Z}=\left(I-Q^{\tau}\right)^{-1} \mathcal{Z}=\left(\sum_{m=0}^{\infty}\left(Q^{\tau}\right)^{m}\right) \mathcal{Z} .
$$

The matrix $S$ as formulated in Eq. 2.24 can also be expressed as an infinite series. The substorage matrix $X$ can then be written as follows:

$$
X=S \mathcal{Z}=\mathcal{R}\left(I-Q^{\tau}\right)^{-1} \mathcal{Z}=(I-L)^{-1} \mathcal{R} \mathcal{Z}=\left(\sum_{m=0}^{\infty} L^{m}\right) \mathcal{R} \mathcal{Z} .
$$

Truncating the infinite geometric series in Eqs. D.2 and D.3 at $m=M$ yields the cumulative transient subthroughflow and substorage matrices at step $M, T^{[M]}=$ $\left(\sum_{m=0}^{M}\left(Q^{\tau}\right)^{m}\right) \mathcal{Z}$ and $X^{[M]}=\left(\sum_{m=0}^{M} L^{m}\right) \mathcal{R} \mathcal{Z}$, respectively. Therefore,

$$
\lim _{M \rightarrow \infty} X^{[M]}=\lim _{M \rightarrow \infty}\left(\sum_{m=0}^{M} L^{m}\right) \mathcal{R} \mathcal{Z}=X .
$$

Similarly, $\lim _{M \rightarrow \infty} T^{[M]}=T$.

\section{Appendix E. The diact flows and storages.}

The input-oriented, simple and composite diact flows are defined, and the derivations for indirect flows and storages are presented in detail in Section 2.6. Parallel derivations for other diact flows and the associated storages generated by these flows are formulated in this section. The alternative path-based formulation of diact flows and storages is given in the Supplementary Materials (Section S3.1). The outputoriented, flow- and storage-based, simple and composite diact flows and storages are formulated further below and in the Supplementary Materials (Section S4.3).

The simple diact flows and storages are defined in Section 2.6. They are explicitly formulated in Section 2.6 and further below. The simple diact flows can be listed as follows:

$$
\begin{array}{rlrl}
\tau_{i_{k}}^{\mathrm{t}}=\tau_{i_{k}}-z_{i_{k}}=\tilde{\tau}_{i_{k}}, & \tau_{i_{k}}^{\mathrm{d}}=f_{i_{k} k_{k}}, & \tau_{i_{k}}^{\mathrm{i}}=\tau_{i_{k}}^{\mathrm{t}}-\tau_{i_{k}}^{\mathrm{d}}, \\
\tau_{i_{k}}^{\mathrm{c}}=\frac{\tau_{i_{i}}-z_{i_{i}}}{\tau_{i_{i}}} \tau_{i_{k}}, & \tau_{i_{k}}^{\mathrm{a}}=\tau_{i_{k}}^{\mathrm{t}}-\tau_{i_{k}}^{\mathrm{c}} .
\end{array}
$$


Note that, the $(i, k)$-element of the simple transfer flow matrix is the intercompartmental subthroughflow at compartment $i$ generated by the environmental input into $k, z_{k}$. That is, in matrix form, $\tilde{T}^{\mathrm{t}}=\tilde{T}$. Let the superscript $\left(^{*}\right)$ stand for any of the diact symbols. The $(i, k)$-elements of the other simple diac flow matrices, $\tilde{T}^{*}=\left(\tau_{i_{k}}^{*}\right)$, represent the direct, indirect, non-cycling, and cycling segments of this simple transfer flow at the terminal compartment $i$. The associated storages generated by these simple diact flows are then represented by the $(i, k)$-elements of the corresponding simple diact storage matrices, $\tilde{X}^{*}=\left(x_{i_{k}}^{*}\right)$.

The steady-state condition and the equivalence of the outward throughflow and subthroughflow intensities in the same direction imply the following proportionalities as formulated in Eq. 2.29, as well as, in matrix form, in Eq. B.3:

$$
\frac{\hat{\tau}_{k_{\ell}}}{\hat{\tau}_{k_{k}}}=\frac{\check{\tau}_{k_{\ell}}}{\check{\tau}_{k_{k}}} \text { and } \quad \frac{x_{i_{\ell}}}{\tau_{i_{\ell}}}=\frac{x_{i}}{\tau_{i}} \text {. }
$$

Equation E.2 implies that composite diact subflow $\tau_{i_{\ell} k_{\ell}}^{*}$ in subsystem $\ell$ parallel to simple diact subflow $\tau_{i_{k} k_{k}}^{*}$, from an input-receiving subcompartment $k_{k}$ to $i_{k}$ in subsystem $k$, and the associated substorage generated by diact subflow $\tau_{i_{\ell} k_{\ell}}^{*}, x_{i_{\ell} k_{\ell}}^{*}$, can be formulated as

$$
\tau_{i_{\ell} k_{\ell}}^{*}=\tau_{i_{k} k_{k}}^{*} \frac{\tau_{k_{\ell}}}{\tau_{k_{k}}} \quad \text { and } \quad x_{i_{\ell} k_{\ell}}^{*}=\tau_{i_{\ell} k_{\ell}}^{*} \frac{x_{i}}{\tau_{i}}
$$

for $i, k=1, \ldots, n$. By parallel subflows, we mean the flows that transit through different subcompartments of the same compartment at the same time. The diact flow and storage from compartment $k$ to $i$ then become

$$
\tau_{i k}^{*}=\sum_{\ell=1}^{n} \tau_{i_{\ell} k_{\ell}}^{*}=n_{i k}^{*} \sum_{\ell=1}^{n} \tau_{k_{\ell}}=n_{i k}^{*} \tau_{k} \quad \text { and } \quad x_{i k}^{*}=r_{i} \tau_{i k}^{*}=s_{i k}^{*} \tau_{k}
$$

where the diact flow and storage distribution factors are

$$
n_{i k}^{*}=\frac{\tau_{i_{k} k_{k}}^{*}}{\tau_{k_{k}}}=\frac{n_{i_{k}}^{*}}{n_{k_{k}}} \quad \text { and } \quad s_{i k}^{*}=r_{i} n_{i k}^{*} .
$$

The scaled and nondimensionalized flows are defined as $n_{i_{k}}^{*}=\tau_{i_{k} k_{k}}^{*} / z_{k}$ and $n_{k_{k}}=$ $\tau_{k_{k}} / z_{k}$. In matrix form, they can be expressed as $\tilde{N}^{*}=\left(n_{i_{k}}^{*}\right)$ and $\mathcal{N}=\operatorname{diag}(N)$. The scaled form in the second equality for $n_{i k}^{*}$ can be used when there is a zero environmental input and, therefore, $\tau_{k_{k}}=0$ for some $k$, as discussed in Section 2.3. The diact flow and storage distribution matrices, $N^{*}=\left(n_{i k}^{*}\right)$ and $S^{*}=\left(s_{i k}^{*}\right)$, can then be written, using Eq. E.5, as follows:

$$
N^{*}=\tilde{T}^{*} \mathrm{~T}^{-1}=\tilde{N}^{*} \mathcal{N}^{-1} \text { and } S^{*}=\mathcal{R} N^{*} .
$$

The simple diact flows and storages can be expressed in terms of the composite diact flows and storages as

$$
\tau_{i_{k}}^{*}=\tau_{i_{k} k_{k}}^{*} \quad \text { and } \quad x_{i_{k}}^{*}=x_{i_{k} k_{k}}^{*} .
$$

That is, the simple diact flows are the composite diact flows from the input-receiving subcompartments to other subcompartments in the same subsystem. The simple diact flow and storage matrices can then be formulated, in terms of the distribution matrices:

$$
\tilde{T}^{*}=N^{*} \mathrm{~T} \quad \text { and } \quad \tilde{X}^{*}=S^{*} \mathrm{~T}=\mathcal{R} \tilde{T}^{*} .
$$


The $\ell^{\text {th }}$ simple diact flow and storage matrices, $\tilde{\mathcal{T}}_{\ell}^{*}$ and $\tilde{\mathcal{X}}_{\ell}^{*}$, for the $\ell^{\text {th }}$ subsystem will also be defined as the diagonal matrices whose diagonal elements are the $\ell^{t h}$ column vector of $\tilde{T}^{*}$ and $\tilde{X}^{*}$, respectively. That is, $\tilde{\mathcal{T}}_{\ell}^{*}=\operatorname{diag}\left(\tilde{T}^{*} \boldsymbol{e}_{\ell}\right)$ and $\tilde{\mathcal{X}}_{\ell}^{*}=\operatorname{diag}\left(\tilde{X}^{*} \boldsymbol{e}_{\ell}\right)$.

The $\ell^{t h}$ composite diact flow and storage matrices, $\mathcal{T}_{\ell}^{*}=\left(\tau_{i_{\ell} k_{\ell}}^{*}\right)$ and $\mathcal{X}_{\ell}^{*}=\left(x_{i_{\ell} k_{\ell}}^{*}\right)$, will be formulated as

$$
T_{\ell}^{*}=N^{*} \mathcal{T}_{\ell} \quad \text { and } \quad X_{\ell}^{*}=S^{*} \mathcal{T}_{\ell}=\mathcal{R} T_{\ell}^{*},
$$

using Eq. E.3. Therefore, the composite diact flow and storage matrices, $T^{*}=\left(\tau_{i k}^{*}\right)$ and $X^{*}=\left(x_{i k}^{*}\right)$, become

$$
T^{*}=N^{*} \mathcal{T}=\sum_{\ell=1}^{n} T_{\ell}^{*} \quad \text { and } \quad X^{*}=S^{*} \mathcal{T}=\mathcal{R} T^{*}=\sum_{\ell=1}^{n} X_{\ell}^{*},
$$

because of Eq. E.4. Note that, the $(i, k)$-element of the composite transfer flow matrix, $\tau_{i k}^{\mathrm{t}}$, is the total flow from compartment $k$ transferred directly or indirectly through other compartments into $i$. The $(i, k)$-elements of the other composite diac flow matrices represent the direct, indirect, non-cycling, and cycling segments of this composite transfer flow at the terminal compartment $i$. The associated storages generated by these composite diact flows are then represented by the $(i, k)$-elements of the corresponding composite diact storage matrices, $x_{i k}^{*}$.

In matrix form, the simple and composite diact flows can be represented at the subcompartmental and compartmental levels as follows:

$$
\begin{aligned}
& \tilde{\mathcal{T}}_{\ell}^{*}=\left[\begin{array}{cccc}
\tau_{1_{\ell}}^{*} & 0 & \cdots & 0 \\
0 & \tau_{2_{\ell}}^{*} & \cdots & 0 \\
\vdots & & \ddots & \vdots \\
0 & 0 & \cdots & \tau_{n_{\ell}}^{*}
\end{array}\right], \quad \tilde{T}^{*}=\left[\begin{array}{ccccc}
\tau_{1_{1}}^{*} & \cdots & \tau_{1_{\ell}}^{*} & \cdots & \tau_{1_{n}}^{*} \\
\tau_{2_{1}}^{*} & \cdots & \tau_{2_{\ell}}^{*} & \cdots & \tau_{2_{n}}^{*} \\
\vdots & \ddots & \vdots & \ddots & \vdots \\
\tau_{n_{1}}^{*} & \cdots & \tau_{n_{\ell}}^{*} & \cdots & \tau_{n_{n}}^{*}
\end{array}\right], \\
& T_{\ell}^{*}=\left[\begin{array}{ccccc}
\tau_{1_{\ell} 1_{\ell}}^{*} & \cdots & \tau_{1_{k} k_{\ell}}^{*} & \cdots & \tau_{1_{\ell} n_{\ell}}^{*} \\
\tau_{2_{\ell} 1_{\ell}}^{*} & \cdots & \tau_{2_{\ell} k_{\ell}}^{*} & \cdots & \tau_{2_{\ell} n_{\ell}}^{*} \\
\vdots & \ddots & \vdots & \ddots & \vdots \\
\tau_{n_{\ell} 1_{\ell}}^{*} & \cdots & \tau_{n_{\ell} k_{\ell}}^{*} & \cdots & \tau_{n_{\ell} n_{\ell}}^{*}
\end{array}\right], \quad T^{*}=\left[\begin{array}{ccccc}
\tau_{11}^{*} & \cdots & \tau_{1 k}^{*} & \cdots & \tau_{1 n}^{*} \\
\tau_{21}^{*} & \cdots & \tau_{2 k}^{*} & \cdots & \tau_{2 n}^{*} \\
\vdots & \ddots & \vdots & \ddots & \vdots \\
\tau_{n 1}^{*} & \cdots & \tau_{n k}^{*} & \cdots & \tau_{n n}^{*}
\end{array}\right] \text {, }
\end{aligned}
$$

where $\tau_{i_{\ell}}^{*}=\tau_{i_{\ell} \ell_{\ell}}^{*}$ and $\tau_{i k}^{*}=\sum_{k=1}^{n} \tau_{i_{\ell} k_{\ell}}^{*}$, as defined in Eqs. E.7 and E.10. The diact storage matrices can be represented similar to these diact flow matrices.

The difference between the composite and simple diact flows, $\tau_{i k}^{*}$ and $\tau_{i_{k}}^{*}$, and associated storages, $x_{i k}^{*}$ and $x_{i_{k}}^{*}$, is that the composite flow and storage from compartment $k$ to $i$ are generated by outward throughflow $\hat{\tau}_{k}$ derived from all environmental inputs, and their simple counterparts from input-receiving subcompartment $k_{k}$ to $i_{k}$ are generated by outward subthroughflow $\hat{\tau}_{k_{k}}$ derived from single environmental input $z_{k}$ (see Fig. 4). In that sense, the composite and simple diact flows and storages measure the influence of one compartment on another induced by all and single environmental inputs, respectively.

Since the simple diact flows and storages are constituents of the corresponding composite diact flows and storages, we have

$$
T^{*} \geq \tilde{T}^{*} \Rightarrow \dot{T}^{*}=T^{*}-\tilde{T}^{*} \geq \mathbf{0} \quad \text { and } \quad X^{*} \geq \tilde{X}^{*} \Rightarrow \dot{X}^{*}=X^{*}-\tilde{X}^{*} \geq \mathbf{0}
$$

These inequalities are defined componentwise. The elements of the $k^{\text {th }}$ column of $\dot{T}^{*}$ and $\dot{X}^{*}$ are the diact flows and storages from compartment $k$ to the others generated 
by all environmental inputs but $z_{k}$. The matrices $\dot{T}^{*}$ and $\dot{X}^{*}$ will accordingly be called complementary diact flow and storage matrices, respectively.

The simple diact throughflow and compartmental storage matrices, $\tilde{\mathcal{T}}^{*}$ and $\tilde{\mathcal{X}}^{*}$, and vectors, $\tilde{\tau}^{*}$ and $\tilde{x}^{*}$, can be defined as

$$
\begin{aligned}
& \tilde{\mathcal{T}}^{*}=\operatorname{diag}\left(\tilde{T}^{*} \mathbf{1}\right) \Rightarrow \tilde{\tau}^{*}=\tilde{\mathcal{T}}^{*} \mathbf{1} \text { and } \\
& \tilde{\mathcal{X}}^{*}=\operatorname{diag}\left(\tilde{X}^{*} \mathbf{1}\right) \Rightarrow \tilde{x}^{*}=\tilde{\mathcal{X}}^{*} \mathbf{1} \text {. }
\end{aligned}
$$

Similar relationships can also be formulated for composite diact throughflow and compartmental storage matrices, $\mathcal{T}^{*}$ and $\mathcal{X}^{*}$, and vectors, $\tau^{*}$ and $x^{*}$.

The relationship between the diact distribution matrices and the corresponding flow and storage matrices can be summarized as follows: by left multiplying the (sub)throughflow matrix, the diact flow and storage distribution matrices yield the corresponding diact (sub)flow and associated (sub)storage matrices. That is,

$$
T^{*}=N^{*} \mathcal{T}, \quad X^{*}=S^{*} \mathcal{T} \quad \text { and } \quad T_{\ell}^{*}=N^{*} \mathcal{T}_{\ell}, \quad X_{\ell}^{*}=S^{*} \mathcal{T}_{\ell} .
$$

Due to their construction, the diact distribution matrices and the corresponding flow and storage matrices are related as follows:

$$
S^{*}=\mathcal{R} N^{*} \quad \Rightarrow \quad X^{*}=\mathcal{R} T^{*} \quad \text { and } \quad X_{\ell}^{*}=\mathcal{R} T_{\ell}^{*} .
$$

similar to Eq. 2.26. The simple counterparts of these relationships for the composite diact flow and storage distribution matrices can also be formulated, as listed in Tables 1 and 2. Note that, for the flow-based diact distribution matrices, the unit of $S^{*}$ is time [t], as the cumulative storage distribution matrix, $S$, and $N^{*}$ is dimensionless, as the cumulative throughflow distribution matrix, $N$.

It is worth emphasizing also that the methodology outlined above for derivations of diact flows and storages can be used to define new transaction types. New simple flow and storage matrices, the corresponding scaled and nondimensionalized flow and storage distribution matrices, as well as the composite flow and storage matrices can be formulated similar to the derivations of the diact flows and storages.

The input-oriented, flow- and storage-based, simple and composite diact flow and storage distribution matrices, as well as the corresponding flow and associated storage matrices are listed in Table 1 and 2 at both compartmental and subcompartmental levels. The storage-based diact flows and storages can also be formulated using Eq. E.46. Additional formulations for each diact transaction type are separately presented below.

E.1. Direct flow and storage. The composite direct flow is defined as $\tau_{i j}^{\mathrm{d}}=f_{i j}$ and, therefore, $T^{\mathrm{d}}=F$. The proportionality given in Eq. E.3 implies that

$$
x_{i_{k} j_{k}}^{\mathrm{d}}=\tau_{i_{k} j_{k}}^{\mathrm{d}} \frac{x_{i}}{\tau_{i}}=f_{i_{k} j_{k}} \frac{x_{i}}{\tau_{i}} \text { and } x_{i j}^{\mathrm{d}}=\sum_{k=1}^{n} x_{i_{k} j_{k}}^{\mathrm{d}}=f_{i j} \frac{x_{i}}{\tau_{i}} .
$$

Defining the direct flow and storage distribution matrices as

$$
N^{\mathrm{d}}=F \mathcal{T}^{-1}=Q^{\tau} \quad \text { and } \quad S^{\mathrm{d}}=\mathcal{R} N^{\mathrm{d}}=\mathcal{R} Q^{\tau}=L \mathcal{R},
$$

the $\ell^{\text {th }}$ composite direct subflow and associated substorage matrices for the $\ell^{\text {th }}$ subsystem, $T_{\ell}^{\mathrm{d}}$ and $X_{\ell}^{\mathrm{d}}$, can be expressed in matrix form as follows:

$$
T_{\ell}^{\mathrm{d}}=N^{\mathrm{d}} \mathcal{T}_{\ell}=F \mathcal{T}^{-1} \mathcal{T}_{\ell}=F_{\ell} \quad \text { and } \quad X_{\ell}^{\mathrm{d}}=S^{\mathrm{d}} \mathcal{T}_{\ell}=\mathcal{R} T_{\ell}^{\mathrm{d}} .
$$


The composite direct flow and storage matrices, $T^{\mathrm{d}}$ and $X^{\mathrm{d}}$, then become

$$
T^{\mathrm{d}}=N^{\mathrm{d}} \mathcal{T}=F=\sum_{\ell=1}^{n} T_{\ell}^{\mathrm{d}} \quad \text { and } \quad X^{\mathrm{d}}=S^{\mathrm{d}} \mathcal{T}=\mathcal{R} T^{\mathrm{d}}=\mathcal{R} F=\sum_{\ell=1}^{n} X_{\ell}^{\mathrm{d}}
$$

The composite direct throughflow and compartmental storage matrices and vectors can also be formulated as

$$
\begin{aligned}
& \mathcal{T}^{\mathrm{d}}=\operatorname{diag}\left(T^{\mathrm{d}} \mathbf{1}\right)=\mathcal{T}-\mathcal{Z} \Rightarrow \tau^{\mathrm{d}}=\mathcal{T}^{\mathrm{d}} \mathbf{1}=\tau-z \quad \text { and } \\
& \mathcal{X}^{\mathrm{d}}=\operatorname{diag}\left(X^{\mathrm{d}} \mathbf{1}\right) \Rightarrow x^{\mathrm{d}}=\mathcal{X}^{\mathrm{d}} \mathbf{1} .
\end{aligned}
$$

E.2. Indirect flow and storage. The simple and composite indirect flows and storages are introduced in Section 2.6. The indirect flow and storage distribution matrices are

$$
N^{\mathrm{i}}=\left(T-\mathcal{Z}-\tilde{T}^{\mathrm{d}}\right) \mathrm{T}^{-1}=(N-I) \mathcal{N}^{-1}-F \mathcal{T}^{-1} \quad \text { and } \quad S^{\mathrm{i}}=\mathcal{R} N^{\mathrm{i}} .
$$

In component form, the indirect flow and storage distribution factors, can be written as

$$
n_{i k}^{\mathrm{i}}=\frac{\tau_{i_{k}}-z_{i_{k}}-f_{i_{k} k_{k}}}{\tau_{k_{k}}}=\frac{n_{i_{k}}-\delta_{i k}}{n_{k_{k}}}-\frac{f_{i k}}{\tau_{k}} \quad \text { and } \quad s_{i k}^{\mathrm{i}}=r_{i} n_{i k}^{\mathrm{i}} .
$$

The $\ell^{\text {th }}$ composite indirect subflow and associated substorage matrices, $T_{\ell}^{\mathrm{i}}$ and $X_{\ell}^{i}$, are formulated in Eq. 2.42 as follows:

$$
T_{\ell}^{\mathrm{i}}=N^{\mathrm{i}} \mathcal{T}_{\ell} \quad \text { and } \quad X_{\ell}^{\mathrm{i}}=S^{\mathrm{i}} \mathcal{T}_{\ell}=\mathcal{R} T_{\ell}^{\mathrm{i}} .
$$

The composite indirect flow and associated storage matrices given in Eq. 2.44 can then be expressed as

$$
T^{\mathrm{i}}=N^{\mathrm{i}} \mathcal{T}=\sum_{\ell=1}^{n} T_{\ell}^{\mathrm{i}} \quad \text { and } \quad X^{\mathrm{i}}=S^{\mathrm{i}} \mathcal{T}=\mathcal{R} T^{\mathrm{i}}=\sum_{\ell=1}^{n} X_{\ell}^{\mathrm{i}} .
$$

E.3. Acyclic flow and storage. The simple acyclic subflow from an inputreceiving subcompartment $k_{k}$ to $i_{k}$ in the $k^{\text {th }}$ subsystem can be formulated as

$$
\tau_{i_{k}}^{\mathrm{a}}=\tau_{i_{k} k_{k}}^{\mathrm{a}}=\tau_{i_{k}}^{\mathrm{t}}-\tau_{i_{k}}^{\mathrm{c}}=\left(\tau_{i_{k}}-z_{i_{k}}\right)-\left(\frac{\tau_{i_{i}}-z_{i_{i}}}{\tau_{i_{i}}}\right) \tau_{i_{k}} .
$$

The composite subflow parallel to $\tau_{i_{k} k_{k}}^{\mathrm{a}}$ in subsystem $\ell$ then becomes

$$
\tau_{i_{\ell} k_{\ell}}^{\mathrm{a}}=\tau_{i_{k} k_{k}}^{\mathrm{a}} \frac{\tau_{k_{\ell}}}{\tau_{k_{k}}}=\left[\frac{\tau_{i_{k}}-z_{i_{k}}}{\tau_{k_{k}}}-\frac{\tau_{i_{i}}-z_{i_{i}}}{\tau_{i_{i}}} \frac{\tau_{i_{k}}}{\tau_{k_{k}}}\right] \tau_{k_{\ell}}
$$

and, therefore, the acyclic flow and storage distribution factors are

$$
n_{i k}^{\mathrm{a}}=\frac{\tau_{i_{k}}-z_{i_{k}}}{\tau_{k_{k}}}-\frac{\tau_{i_{i}}-z_{i_{i}}}{\tau_{i_{i}}} \frac{\tau_{i_{k}}}{\tau_{k_{k}}}=\frac{1}{n_{i_{i}}} n_{i_{k}} \frac{1}{n_{k_{k}}}-\frac{\delta_{i k}}{n_{k_{k}}} \quad \text { and } \quad s_{i k}^{\mathrm{a}}=r_{i} n_{i k}^{\mathrm{a}} .
$$

The acyclic flow and storage distribution matrices can be formulated accordingly:

$$
N^{\mathrm{a}}=\left(\mathcal{N}^{-1} N-I\right) \mathcal{N}^{-1} \quad \text { and } \quad S^{\mathrm{a}}=\mathcal{R} N^{\mathrm{a}} .
$$


The $\ell^{t h}$ composite acyclic subflow and associated substorage matrices can be written in matrix form as follows:

$$
T_{\ell}^{\mathrm{a}}=N^{\mathrm{a}} \mathcal{T}_{\ell}=\left(\mathcal{N}^{-1} N-I\right) \mathcal{N}^{-1} \mathcal{T}_{\ell} \quad \text { and } \quad X_{\ell}^{\mathrm{a}}=S^{\mathrm{a}} \mathcal{T}_{\ell}=\mathcal{R} T_{\ell}^{\mathrm{a}} .
$$

The composite acyclic flow and associated storage matrices become

$$
T^{\mathrm{a}}=N^{\mathrm{a}} \mathcal{T}=\sum_{\ell=1}^{n} T_{\ell}^{\mathrm{a}} \quad \text { and } \quad X^{\mathrm{a}}=S^{\mathrm{a}} \mathcal{T}=\mathcal{R} T^{\mathrm{a}}=\sum_{\ell=1}^{n} X_{\ell}^{\mathrm{a}} .
$$

E.4. Cycling flow and storage. The simple cycling subflow from an inputreceiving subcompartment $k_{k}$ to $i_{k}$ in the $k^{t h}$ subsystem can be formulated, using Eq. E.34, as

$$
\tau_{i_{k}}^{\mathrm{c}}=\tau_{i_{k} k_{k}}^{\mathrm{c}}=\frac{\tau_{i_{i}}-z_{i_{i}}}{\tau_{i_{i}}} \tau_{i_{k}} .
$$

The composite subflow parallel to $\tau_{i_{k} k_{k}}^{\mathrm{c}}$ in subsystem $\ell$ then becomes

$$
\tau_{i_{\ell} k_{\ell}}^{\mathrm{c}}=\tau_{i_{k} k_{k}}^{\mathrm{c}} \frac{\tau_{k_{\ell}}}{\tau_{k_{k}}}=\frac{\tau_{i_{i}}-z_{i_{i}}}{\tau_{i_{i}}} \frac{\tau_{i_{k}}}{\tau_{k_{k}}} \tau_{k_{\ell}}
$$

and, therefore, the cycling flow and storage distribution factors are

$$
n_{i k}^{\mathrm{c}}=\frac{\tau_{i_{i}}-z_{i_{i}}}{\tau_{i_{i}}} \frac{\tau_{i_{k}}}{\tau_{k_{k}}}=\frac{n_{i_{k}}}{n_{k_{k}}}-\frac{1}{n_{i_{i}}} n_{i_{k}} \frac{1}{n_{k_{k}}} \quad \text { and } \quad s_{i k}^{\mathrm{c}}=r_{i} n_{i k}^{\mathrm{c}} .
$$

The cycling flow and storage distribution matrices can be formulated accordingly:

$$
N^{\mathrm{c}}=\left(N-\mathcal{N}^{-1} N\right) \mathcal{N}^{-1} \quad \text { and } \quad S^{\mathrm{c}}=\mathcal{R} N^{\mathrm{c}} .
$$

The $\ell^{\text {th }}$ composite cycling subflow and substorage matrices can be expressed in matrix form as

$$
T_{\ell}^{\mathrm{c}}=N^{\mathrm{c}} \mathcal{T}_{\ell}=\left(N-\mathcal{N}^{-1} N\right) \mathcal{N}^{-1} \mathcal{T}_{\ell} \quad \text { and } \quad X_{\ell}^{\mathrm{c}}=S^{\mathrm{c}} \mathcal{T}_{\ell}=\mathcal{R} T_{\ell}^{\mathrm{c}} .
$$

The composite cycling flow and storage matrices then become

$$
T^{\mathrm{c}}=N^{\mathrm{c}} \mathcal{T}=\sum_{\ell=1}^{n} T_{\ell}^{\mathrm{c}} \quad \text { and } \quad X^{\mathrm{c}}=S^{\mathrm{c}} \mathcal{T}=\mathcal{R} T^{\mathrm{c}}=\sum_{\ell=1}^{n} X_{\ell}^{\mathrm{c}} .
$$

The simple cycling subflow from an input-receiving subcompartment $i_{i}$ to itself can, alternatively, be formulated as follows:

$$
\tau_{i_{i}}^{\mathrm{c}}=\tau_{i_{i} i_{i}}^{\mathrm{i}}=\tau_{i_{i} i_{i}}^{\mathrm{t}}=\sum_{j=1}^{n} f_{i_{i} j_{i}}=\check{\tau}_{i_{i}}-z_{i} .
$$

The matrix form of this relationship is given in Eq. 2.66. Note that this relationship is equivalent to Eq. E.27 for $k=i$. The simple cycling flow at subcompartment $i_{k}$, $\tau_{i_{k}}^{\mathrm{c}}$, parallel to $\tau_{i_{i}}^{\mathrm{c}}$ in the $k^{\text {th }}$ subsystem then becomes

$$
\tau_{i_{k}}^{\mathrm{c}}=\left(\check{\tau}_{i_{i}}-z_{i}\right) \frac{\hat{\tau}_{i_{k}}}{\hat{\tau}_{i_{i}}}
$$




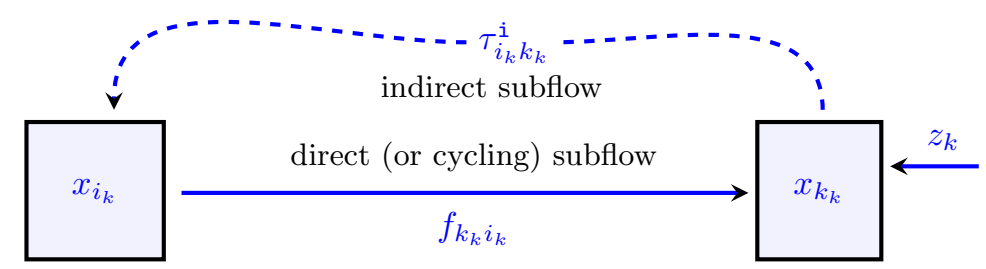

FIG. 10. Schematic representation for the complementary nature of the simple indirect and cycling flows in the kth subsystem. The composite direct subflow, $f_{k_{k} i_{k}}$, is represented by solid arrow. This subflow also contributes to the simple cycling flow at subcompartment $k_{k}$. The simple indirect flow, $\tau_{i_{k} k_{k}}^{i}$, through other compartments (not shown) is represented by dashed arrows.

Consequently, the simple cycling flow at compartment $i, \tau_{i}^{\mathrm{c}}=\tau_{i i}^{\mathrm{c}}$, becomes

$$
\tau_{i}^{\mathrm{c}}=\sum_{k=1}^{n} \tau_{i_{k}}^{\mathrm{c}}=\sum_{k=1}^{n}\left(\tau_{i_{i}}-z_{i}\right) \frac{\tau_{i_{k}}}{\tau_{i_{i}}}=\frac{\tau_{i_{i}}-z_{i}}{\tau_{i_{i}}} \sum_{k=1}^{n} \tau_{i_{k}}=\frac{\tau_{i_{i}}-z_{i}}{\tau_{i_{i}}} \tau_{i}=n_{i}^{\mathrm{c}} \tau_{i}
$$

where the simple cycling flow and storage distribution factors are

$$
n_{i}^{\mathrm{c}}=\frac{\tau_{i_{i}}-z_{i}}{\tau_{i_{i}}}=\frac{n_{i_{i}}-1}{n_{i_{i}}} \text { and } s_{i}^{\mathrm{c}}=r_{i} n_{i}^{\mathrm{c}}
$$

where $\tau_{i_{i}} \neq 0$. The reflexivity of the simple cycling subflow formulated in Eq. E.33 is also manifested in the distribution factors: $n_{i}^{\mathrm{c}}=n_{i i}^{\mathrm{i}}=n_{i i}^{\mathrm{t}}$, as also discussed in Eq. 2.65 (see Fig. 10).

The new diagonal simple cycling flow and storage distribution matrices, $\mathcal{N}^{c}=$ $\operatorname{diag}\left(\left[n_{1}^{\mathrm{c}}, \ldots, n_{n}^{\mathrm{c}}\right]\right)$ and $\mathcal{S}^{\mathrm{c}}=\operatorname{diag}\left(\left[s_{1}^{\mathrm{c}}, \ldots, s_{n}^{\mathrm{c}}\right]\right)$, then become

$$
\mathcal{N}^{\mathrm{c}}=(\mathrm{T}-\mathcal{Z}) \mathrm{T}^{-1}=(\mathcal{N}-I) \mathcal{N}^{-1} \text { and } \quad \mathcal{S}^{\mathrm{c}}=\mathcal{R} \mathcal{N}^{\mathrm{c}}
$$

The $\ell^{\text {th }}$ diagonal simple cycling subflow and associated substorage matrices, $\tilde{\mathcal{T}}_{\ell}^{\mathrm{c}}=$ $\operatorname{diag}\left(\left[\tau_{1_{\ell}}^{\mathrm{c}}, \cdots, \tau_{n_{\ell}}^{\mathrm{c}}\right]\right)$ and $\tilde{\mathcal{X}}_{\ell}^{\mathrm{c}}=\operatorname{diag}\left(\left[x_{1_{\ell}}^{\mathrm{c}}, \cdots, x_{n_{\ell}}^{\mathrm{c}}\right]\right)$, can be expressed in the following matrix form

$$
\tilde{\mathcal{T}}_{\ell}^{\mathrm{c}}=\mathcal{N}^{\mathrm{c}} \mathcal{T}_{\ell} \quad \text { and } \quad \tilde{\mathcal{X}}_{\ell}^{\mathrm{c}}=\mathcal{S}^{\mathrm{c}} \mathcal{T}_{\ell}=\mathcal{N}^{\mathrm{c}} \mathcal{X}_{\ell}=\mathcal{R} \tilde{\mathcal{T}}_{\ell}^{\mathrm{c}} .
$$

The simple cycling subflow and associated storage matrices can then be formulated as

$$
\tilde{T}^{\mathrm{c}}=\mathcal{N}^{\mathrm{c}} T \quad \text { and } \quad \tilde{X}^{\mathrm{c}}=\mathcal{S}^{\mathrm{c}} T=\mathcal{N}^{\mathrm{c}} X .
$$

Note that the two equivalent versions of the single cycling flow and storage distribution matrices are related as follows:

$$
\tilde{T}^{\mathrm{c}}=\mathcal{N}^{\mathrm{c}} T=N^{\mathrm{c}} \mathrm{T} \quad \text { and } \quad \tilde{X}^{\mathrm{c}}=\mathcal{S}^{\mathrm{c}} T=S^{\mathrm{c}} \mathrm{T} .
$$

Moreover,

$$
\tilde{\mathcal{T}}_{\ell}^{c}=\operatorname{diag}\left(\tilde{T}^{c} \boldsymbol{e}_{\ell}\right)=\operatorname{diag}\left(T_{\ell}^{c}\right) \quad \text { and } \quad \tilde{\mathcal{X}}_{\ell}^{c}=\operatorname{diag}\left(\tilde{X}^{c} \boldsymbol{e}_{\ell}\right)=\operatorname{diag}\left(X_{\ell}^{c}\right) .
$$


TABLE 2

The input-oriented, storage-based diact flow and storage distribution and the simple and composite diact (sub)flow and (sub)storage matrices. The superscript $\left(^{*}\right)$ in each equation represents any of the diact symbols.

\begin{tabular}{|c|c|c|c|c|}
\hline diact & flow and storage distribution & matrices & flows & storages \\
\hline$d$ & $S^{\mathrm{d}, x}=F \mathcal{X}^{-1}$ & & & \\
\hline$i$ & $S^{i, x}=\left(\mathcal{R}^{-1} S-I\right) \mathcal{S}^{-1}-F \mathcal{X}^{-1}$ & & $T^{*}=S^{*, x} \mathcal{X}$ & $X^{*}=N^{*, x} \mathcal{X}$ \\
\hline a & $S^{\mathrm{a}, x}=\left(\mathcal{S}^{-1} S-I\right) \mathcal{S}^{-1}$ & $N^{*, x}=\mathcal{R} S^{*, x}$ & $T_{\ell}^{*}=S^{*, x} \mathcal{X}_{\ell}$ & $X_{\ell}^{*}=N^{*, x} \mathcal{X}_{\ell}$ \\
\hline$c$ & $S^{\mathrm{c}, x}=\left(\mathcal{R}^{-1} S-\mathcal{S}^{-1} S\right) \mathcal{S}^{-1}$ & & $\tilde{T}^{*}=S^{*, x} \mathrm{X}$ & $\tilde{X}^{*}=N^{*, x} \mathrm{X}$ \\
\hline t & $S^{\mathrm{t}, x}=\left(\mathcal{R}^{-1} S-I\right) \mathcal{S}^{-1}$ & & & \\
\hline
\end{tabular}

E.5. Transfer flow and storage. The simple transfer subflow from an inputreceiving subcompartment $k_{k}$ to $i_{k}$ can be expressed as follows:

$$
\tau_{i_{k}}^{\mathrm{t}}=\tau_{i_{k} k_{k}}^{\mathrm{t}}=\sum_{j=1}^{n} f_{i_{k} j_{k}}=\check{\tau}_{i_{k}}-z_{i_{k}}
$$

Therefore, the transfer flow and storage distribution factors become

$$
n_{i k}^{\mathrm{t}}=\frac{\tau_{i_{k}}-z_{i_{k}}}{\tau_{k_{k}}}=\frac{n_{i_{k}}-\delta_{i k}}{n_{k_{k}}} \text { and } s_{i k}^{\mathrm{t}}=r_{i} n_{i k}^{\mathrm{t}} .
$$

Note that, although the derivation rationales, notations, and terminologies are different, the alternative simple cycling and transfer flow distribution factors, $n_{i}^{c}$ and $n_{i k}^{\mathrm{t}}$, formulated in Eqs. E.36 and E.42, in terms of the elements of $N$, are equivalent to those defined by [20] and [45], respectively.

The transfer flow and storage distribution matrices, $N^{\mathrm{t}}=\left(n_{i k}^{\mathrm{t}}\right)$ and $S^{\mathrm{t}}=\left(s_{i k}^{\mathrm{t}}\right)$, can be expressed as

$$
N^{\mathrm{t}}=(N-I) \mathcal{N}^{-1} \quad \text { and } \quad S^{\mathrm{t}}=\mathcal{R} N^{\mathrm{t}}=\mathcal{R}(N-I) \mathcal{N}^{-1} .
$$

The $\ell^{\text {th }}$ composite transfer subflow and substorage matrices $T_{\ell}^{\mathrm{t}}$ and $X_{\ell}^{\mathrm{t}}$ will be formulated as

$$
T_{\ell}^{\mathrm{t}}=N^{\mathrm{t}} \mathcal{T}_{\ell} \quad \text { and } \quad X_{\ell}^{\mathrm{t}}=S^{\mathrm{t}} \mathcal{T}_{\ell}=\mathcal{R} T_{\ell}^{\mathrm{t}} .
$$

The composite transfer flow and associated storage matrix measures become

$$
T^{\mathrm{t}}=N^{\mathrm{t}} \mathcal{T}=\sum_{\ell=1}^{n} T_{\ell}^{\mathrm{t}} \quad \text { and } \quad X^{\mathrm{t}}=S^{\mathrm{t}} \mathcal{T}=\mathcal{R} T^{\mathrm{t}}=\sum_{\ell=1}^{n} T_{\ell}^{\mathrm{t}} .
$$

The simple transfer flow and storage matrices, $\tilde{T}^{\mathrm{t}}$ and $\tilde{X}^{\mathrm{t}}$, are also defined as follows:

$$
\tilde{T}^{\mathrm{t}}=N^{\mathrm{t}} \mathrm{T}=\tilde{T}=T-\mathcal{Z} \quad \text { and } \quad \tilde{X}^{\mathrm{t}}=S^{\mathrm{t}} \mathrm{T}=\mathcal{R} \tilde{T}^{\mathrm{t}}=\tilde{X}=X-\mathcal{R} \mathcal{Z} .
$$

E.6. Storage-based diact transactions. Using the relationships formulated in Eq. 2.26 and the fact that

$$
\mathcal{S}=\mathcal{R} \mathcal{N} \Rightarrow \mathcal{N}^{-1}=\mathcal{S}^{-1} \mathcal{R} \quad \text { and } \quad \mathcal{X}=\mathcal{R} \mathcal{T} \Rightarrow \mathcal{T}^{-1}=\mathcal{X}^{-1} \mathcal{R}
$$


the diact flows and storages formulated in Table 1 can be expressed in terms of storage distribution matrix, $S$. The composite acyclic flow and storage distribution matrices, for example, become

$$
N^{\mathrm{a}}=\left(\mathcal{S}^{-1} S-I\right) \mathcal{S}^{-1} \mathcal{R} \text { and } \quad S^{\mathrm{a}}=\mathcal{R} N^{\mathrm{a}}=\mathcal{R}\left(\mathcal{S}^{-1} S-I\right) \mathcal{S}^{-1} \mathcal{R} .
$$

Therefore,

$$
T^{\mathrm{a}}=N^{\mathrm{a}} \mathcal{T}=\left(\mathcal{S}^{-1} S-I\right) \mathcal{S}^{-1} \mathcal{R} \mathcal{T}=S^{\mathrm{a}, x} \mathcal{X} \quad \text { and } \quad X^{\mathrm{a}}=S^{\mathrm{a}} \mathcal{T}=N^{\mathrm{a}, x} \mathcal{X},
$$

where the storage-based composite acyclic flow and storage distribution matrices can respectively be defined as follows:

$$
S^{\mathrm{a}, x}=\left(\mathcal{S}^{-1} S-I\right) \mathcal{S}^{-1} \quad \text { and } \quad N^{\mathrm{a}, x}=\mathcal{R} S^{\mathrm{a}, x} .
$$

All the other input-oriented, storage-based diact flow and storage distribution matrices, as well as the corresponding simple and composite flow and storage matrices can be formulated similarly, as listed in Table 2 .

\section{Appendix F. Output-oriented system analysis.}

The output-oriented system analysis is introduced by $[29,2]$ in economics. Compartmental systems can be analyzed based on both environmental inputs and outputs. In the context of the proposed methodology, the output-oriented system analysis and the duality of the input- and output-oriented analyses through novel similarity relationships are introduced in this section. The output-oriented analysis backtracks outputs instead of forward tracking of inputs as the input-oriented analysis requires. The details of the derivation of output-oriented analysis are presented in the Supplementary Materials (Section S4).

The main difference between input- and output-oriented analysis in the proposed framework is that, in the latter case, the flow regime is conceptually reversed. That is, the system now becomes "driven" backward in time by outputs rather than forward in time by inputs (consider all arrows reversed in Fig. 1). Therefore, the terms input and inward in the setting of the input-oriented analysis of the original system need to be interpreted as output and outward in this case. More specifically, flow regime of the output-oriented analysis can be expressed as

$$
\bar{F}=F^{T}, \quad \overline{\mathcal{Z}}=\mathcal{Y}, \quad \text { and } \quad \overline{\mathcal{Y}}=\mathcal{Z} .
$$

To distinguish input- and output-oriented quantities, we use the bar notation over the output-oriented ones.

The output-oriented analysis in reference to the input-oriented analysis is an inverse problem formulation in mathematical terms and is not well-defined for all classes of dynamical systems. Using the proposed system partitioning formulation, however, it is straightforward to formulate the output-oriented analysis at steady state. In this section, we will develop the output-oriented counterparts of the input-oriented measures and, more importantly, will also show the duality of the input- and outputoriented analyses through similarity relationships between pairs of corresponding matrix measures.

At steady state, inward and outward throughflows at each compartment are equal, so the reversal of the flow regime does not change neither the throughflows nor the associated storages generated by the throughflows. We, therefore, have the following 
relationships at steady state, in addition to the ones given in Eq. 2.14:

$$
\begin{array}{cll}
\bar{\tau}=\check{\bar{\tau}}=\hat{\bar{\tau}} & \text { and } & \bar{T}=\check{\bar{T}}=\hat{\bar{T}} \\
\tau=\bar{\tau} & \Longrightarrow & \mathcal{T}=\operatorname{diag}(\tau)=\operatorname{diag}(\bar{\tau})=\overline{\mathcal{T}} \\
x=\bar{x} & \Longrightarrow & \mathcal{X}=\operatorname{diag}(x)=\operatorname{diag}(\bar{x})=\overline{\mathcal{X}}
\end{array}
$$

where $\check{\bar{\tau}}$ and $\hat{\bar{\tau}}$ are the output-oriented inward and outward throughflow vectors, respectively. These relationships also imply that

$$
\overline{\mathcal{R}}=\overline{\mathcal{X}} \overline{\mathcal{T}}^{-1}=\mathcal{X} \mathcal{T}^{-1}=\mathcal{R}
$$

On the other hand, since environmental inputs and outputs are not equal in general, their distribution and organization within the system, that is the input- and outputoriented subthroughflow and substorage matrices, are not the same. Therefore,

$$
X \neq \bar{X} \quad \text { and } \quad T \neq \bar{T} \quad \text { if } \quad z \neq y .
$$

Note, however, that

$$
x=X \mathbf{1}=\bar{X} \mathbf{1}=\bar{x} \quad \text { and } \quad \tau=T \mathbf{1}=\bar{T} \mathbf{1}=\bar{\tau},
$$

as also formulated in Eq. F.2. These relationships imply that

$$
\sigma^{x}=\mathbf{1}^{T} x=\mathbf{1}^{T} X \mathbf{1}=\mathbf{1}^{T} \bar{x}=\bar{\sigma}^{x} \quad \text { and } \quad \sigma^{\tau}=\mathbf{1}^{T} \tau=\mathbf{1}^{T} T \mathbf{1}=\mathbf{1}^{T} \bar{\tau}=\bar{\sigma}^{\tau}
$$

where $\sigma^{x}$ and $\sigma^{\tau}$ will be called the total system storage and throughflow, respectively. The total system storage and throughflow are the same in either orientation, as indicated in Eq. F.5.

In parallel to the input-oriented static solutions given in Eq. 2.12, the outputoriented substorage matrix and initial stocks, $\bar{X}$ and $\bar{x}_{0}$, at steady state become

$$
\begin{aligned}
\bar{X} & =-\bar{A}^{-1} \overline{\mathcal{Z}}=\overline{\mathcal{X}}(\overline{\mathcal{T}}-\bar{F})^{-1} \overline{\mathcal{Z}} \\
& =-\bar{A}^{-1} \mathcal{Y}=\mathcal{X}\left(\mathcal{T}-F^{T}\right)^{-1} \mathcal{Y} \quad \text { and } \quad \bar{x}_{0}=\mathbf{0}
\end{aligned}
$$

where

$$
\bar{A}=(\bar{F}-\overline{\mathcal{T}}) \overline{\mathcal{X}}^{-1}=\left(F^{T}-\mathcal{T}\right) \mathcal{X}^{-1}=F^{T} \mathcal{X}^{-1}-\mathcal{R}^{-1}
$$

The output-oriented substorage and subthroughflow matrices and initial subthroughflow vector can then be written as

$$
\begin{aligned}
& \check{\bar{T}}=\mathcal{Y}+F^{T} \mathcal{X}^{-1} \bar{X}, \\
& \hat{\bar{T}}=(\mathcal{Z}+\operatorname{diag}(F \mathbf{1})) \mathcal{X}^{-1} \bar{X}=\mathcal{T} \mathcal{X}^{-1} \bar{X}=\mathcal{R}^{-1} \bar{X}, \\
& \bar{\tau}_{0}=\check{\bar{\tau}}_{0}=\hat{\bar{\tau}}_{0}=\mathbf{0} .
\end{aligned}
$$

Therefore, the static output-oriented substorage and subthroughflow matrices, $\bar{X}$ and $\bar{T}$, obtained by the output-oriented system partitioning methodology can be expressed by the relationships given in Eqs. F.6 and F.7. The $(i, k)$-elements of these matrices, $\bar{x}_{i_{k}}$ and $\bar{\tau}_{i_{k}}$, represent the storage in and throughflow at compartment $i$ destined to exit the system as output from compartment $k$. Therefore, the output-oriented system partitioning enables partitioning the composite compartmental flows and storages into 
TABLE 3

The output-oriented, flow-based diact flow and storage distribution and the simple and composite diact (sub)flow and (sub)storage matrices. The superscript $\left(^{*}\right)$ in each equation represents any of the diact symbols.

\begin{tabular}{|c|c|c|c|c|}
\hline diact & flow and storage distributio & matrices & flows & storages \\
\hline $\mathrm{d}$ & $\bar{N}^{\mathrm{d}}=F^{T} \mathcal{T}^{-1}$ & & & \\
\hline$i$ & $\bar{N}^{\mathrm{i}}=(\bar{N}-I) \mathcal{N}^{-1}-F^{T} \mathcal{T}^{-1}$ & & $\bar{T}^{*}=\bar{N}^{*} \mathcal{T}$ & $\bar{X}^{*}=\bar{S}^{*} \mathcal{T}$ \\
\hline$a$ & $\bar{N}^{\mathrm{a}}=\left(\mathcal{N}^{-1} \bar{N}-I\right) \mathcal{N}^{-1}$ & $\bar{S}^{*}=\mathcal{R} \bar{N}^{*}$ & $\bar{T}_{\ell}^{*}=\bar{N}^{*} \overline{\mathcal{T}}_{\ell}$ & $\bar{X}_{\ell}^{*}=\bar{S}^{*} \overline{\mathcal{T}}_{\ell}$ \\
\hline c & $\bar{N}^{c}=\left(\bar{N}-\mathcal{N}^{-1} \bar{N}\right) \mathcal{N}^{-1}$ & & $\tilde{\bar{T}}^{*}=\bar{N}^{*} \overline{\mathrm{T}}$ & $\tilde{\bar{X}}^{*}=\bar{S}^{*} \overline{\mathrm{T}}$ \\
\hline $\mathrm{t}$ & $\bar{N}^{\mathrm{t}}=(\bar{N}-I) \mathcal{N}^{-1}$ & & & \\
\hline
\end{tabular}

constituent subcompartmental segments destined to exit the system as environmental outputs separately from each compartment.

Similar to the input-oriented scaling introduced in Section 2.3, all but the initial subsystems can be scaled by corresponding positive environmental output, $y_{k}>0(\mathcal{Y}$ is invertible), to analyze the system behavior per unit output. The output-oriented cumulative storage and throughflow distribution matrices, $\bar{S}$ and $\bar{N}$, can, therefore, be formulated as follows:

$$
\bar{S}=\bar{X} \mathcal{Y}^{-1}=\mathcal{X}\left(\mathcal{T}-F^{T}\right)^{-1} \quad \text { and } \quad \bar{N}=\bar{T} \mathcal{Y}^{-1}=\left(I-F^{T} \mathcal{T}^{-1}\right)^{-1} .
$$

The derivation of this formulation is presented in the Supplementary Materials (Section S4.1). All relationships formulated for the input-oriented analysis in the present paper can be extended to output-oriented analysis. The output-oriented counterparts of the relationships formulated in Eq. 2.26, for example, become

$$
\bar{T}=\mathcal{R}^{-1} \bar{X} \Rightarrow \bar{S}=\mathcal{R} \bar{N} .
$$

\section{Appendix G. Output-oriented diact flows and storages.}

The output-oriented diact flow and storage distribution matrices and the simple and composite flows and storages are listed in Table 3 at both compartmental and subcompartmental levels. Similar to the input-oriented, storage-based diact flows and storages introduced in Section E.6, the output-oriented storage-based transactions are also formulated in the Supplementary Materials (Section S4).

Some important relationships between the input- and output-oriented diact flows and storages are also presented in the Supplementary Materials. For example, it is shown that, in either orientation, the simple cycling throughflows and storages are the same, and the acyclic throughflows and storages are related as follows:

$$
\tilde{\tau}^{\mathrm{c}}=\tilde{\bar{\tau}}^{\mathrm{c}}, \quad \tilde{x}^{\mathrm{c}}=\tilde{\tilde{x}}^{\mathrm{c}} \quad \text { and } \quad \tilde{\tau}^{\mathrm{a}}+z=\tilde{\tilde{\tau}}^{\mathrm{a}}+y, \quad \tilde{x}^{\mathrm{a}}+\mathcal{R} z=\tilde{\bar{x}}^{\mathrm{a}}+\mathcal{R} y .
$$

In other words, the amount of environmental inputs that transit through the system compartments are the same in both orientations, as well as the remaining portion of the inputs cycling within the system.

It is also discussed that the input-oriented transient subflows at the terminal and the output-oriented transient subflows at the initial compartment along a linear flow path are the same. Along all possible flow paths, it can then be shown also that

$$
T^{\mathrm{d}}=\left(\bar{T}^{\mathrm{d}}\right)^{T} \quad \text { and } \quad T^{\mathrm{a}}=\left(\bar{T}^{\mathrm{a}}\right)^{T} .
$$

These relationships, however, are not true for the corresponding storages, that is $X^{\mathrm{d}} \neq\left(\bar{X}^{\mathrm{d}}\right)^{T}$ and $X^{\mathrm{a}} \neq\left(\bar{X}^{\mathrm{a}}\right)^{T}$, because of the differences in the compartmental residence times. 


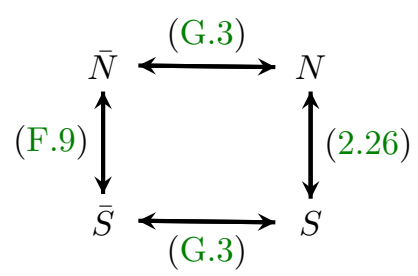

FIG. 11. Relationships between the input- and output-oriented storage and flow distribution matrix measures. The numbers refer to the equation numbers formulating the relationships.

G.1. Duality of the input and output-oriented analyses. The following relationships between the input- and output-oriented flow and storage distribution matrices can be derived using Eqs. 2.22, 2.23, and F.8:

$$
N \mathcal{T}=\mathcal{T} \bar{N}^{T} \quad \text { and } \quad S \mathcal{X}=\mathcal{X} \bar{S}^{T} .
$$

They can be expressed in component form as

$$
\frac{n_{i_{k}}}{\bar{n}_{k_{i}}}=\frac{\tau_{i}}{\tau_{k}} \quad \text { and } \quad \frac{s_{i_{k}}}{\bar{s}_{k_{i}}}=\frac{x_{i}}{x_{k}} .
$$

In terms of linear algebra, Eq. G.3 indicates that the pairs of matrices $\left(S, \bar{S}^{T}\right)$ and $\left(N, \bar{N}^{T}\right)$ are similar to each other, with the same set of eigenvalues. Using Eqs. 2.26 and G.3, the similarity relationships for matrix measures $X, \bar{X}, T$, and $\bar{T}$ can also be expressed as follows:

$$
\begin{array}{lll}
X \mathcal{X}=\mathcal{X}\left(\bar{S}^{T} \mathcal{Z}\right) & \text { and } & \bar{X} \mathcal{X}=\mathcal{X}\left(S^{T} \mathcal{Y}\right) \\
T \mathcal{T}=\mathcal{T}\left(\bar{N}^{T} \mathcal{Z}\right) & \text { and } & \bar{T} \mathcal{T}=\mathcal{T}\left(N^{T} \mathcal{Y}\right)
\end{array}
$$

The formulas in Eq. G.5 can be used to express the relationships between input- and output-oriented substorage and subthroughflow matrices:

$$
X=\left(\mathcal{X} \mathcal{Y}^{-1}\right) \bar{X}^{T}\left(\mathcal{Z} \mathcal{X}^{-1}\right) \quad \text { and } \quad T=\left(\mathcal{T} \mathcal{Y}^{-1}\right) \bar{T}^{T}\left(\mathcal{Z} \mathcal{T}^{-1}\right)
$$

Due to these relationships, componentwise for subcompartments $i_{i}$, we have

$$
\frac{\tau_{i_{i}}}{\bar{\tau}_{i_{i}}}=\frac{z_{i}}{y_{i}}=\frac{x_{i_{i}}}{\bar{x}_{i_{i}}}
$$

which is consistent with Eqs. F.3 and G.4 as well.

The relationships introduced in this section imply that, two of the four matrix measures, $S, \bar{S}, N$, and $\bar{N}$ are redundant, as they can be derived from the other two (see Fig. 11). As examples:

$$
\bar{S}=\mathcal{X} N^{T} \mathcal{T}^{-1} \quad \text { and } \quad N=\mathcal{R} \mathcal{X} \bar{S}^{T} \mathcal{X}^{-1} .
$$

Using the duality relationships, the output-oriented distribution matrices in Table 3 can also be written in terms of the input-oriented matrix measures.

Acknowledgments. The author would like to thank Hasan Coskun for useful discussions and his helpful comments that improved the manuscript. 


\title{
STATIC ECOLOGICAL SYSTEM ANALYSIS
}

\section{HUSEYIN COSKUN}

\author{
A Holistic Analysis of Compartmental Systems
}

\section{SUPPLEMENTARY MATERIALS}

\section{S1. System partitioning methodology.}

The system partitioning methodology explicitly and analytically partitions ecological systems into mutually exclusive and exhaustive subsystems, each driven by a single environmental input, that are running within the original system and have the same structures and dynamics as the original system itself.

Dynamic system partitioning methodology for ecological systems has recently introduced by [10], based on a recent theory of nonlinear system decomposition [12]. The input- and output-oriented static system partitioning methodologies are formulated in the present paper in Section 2.2 and Appendix F, respectively.

\section{S2. Subsystem partitioning methodology.}

The dynamic subsystem partitioning methodology is introduced by [10, 12] for further decomposition or segmentation of subsystems along a given set of mutually exclusive and exhaustive directed subflow paths. This partitioning is similar to the dynamic system partitioning based on environmental inputs as outlined above. The static version of this dynamic subsystem partitioning methodology is introduced in Section 2.4. Additional relationships are formulated in Section S2.3 further below. The subsystem partitioning methodology for the output-oriented analysis is also introduced in Section S4.2.

S2.1. Subflow paths. The formulation of the dynamic and static subsystem partitioning methodologies is based on the concept of directed subflow paths, which will be detailed below [12].

A link will be defined as the connection between two system compartments that represents direct transactions between them. A link constitutes a step along a given flow path from one subcompartment to another. A directed subflow path in a subsystem will then be defined as a chain of connected links initiated at one subcompartment and ending in another of the same subsystem. The number of links or steps along a directed subflow path is called the length of the pathway. The connection of a subflow path to the ambient subsystem is defined as the initial and only subcompartment on the path that receives inflow. The transient subflow and substorage computations along a subflow path must start at its connection. The link to the connection which represents the only inflow into the path will be called the local input and the source of this input will be called the local source. The outflow from the terminal subcompartment of the path will be called the local output. The environment can be taken as the local source or terminal subcompartment.

A subflow path $j_{k} \rightsquigarrow \ell_{k} \rightsquigarrow n_{k} \rightsquigarrow \cdots$ in subsystem $k$ with connection $j_{k}$ and the local source $i_{k}$ will be represented by $i_{k} \mapsto j_{k}^{*} \rightsquigarrow \ell_{k} \rightsquigarrow n_{k} \rightsquigarrow \cdots$. The connection is marked with red superscript $(*)$, and the local input with red arrow. A subflow link that does not directly contribute to the particular subflow or substorage in question will be represented by $(\rightsquigarrow)$ symbol. If the local input or output are external input or output, the corresponding subcompartments on the pathway will be denoted by $0_{k}$. 
Therefore, $0_{k}$ indicates that the local input is external input $z_{k}$, and, since there is no external input for the initial subsystem, $0_{0}$ indicates that the local input is zero. Assuming that the terminal subcompartment of the path partially defined above is $v_{k}$, the complete subflow path will be represented by $p_{v_{k} j_{k}}=i_{k} \mapsto j_{k}^{*} \rightsquigarrow \ell_{k} \rightsquigarrow n_{k} \rightsquigarrow$ $\cdots \rightarrow v_{k}$. If the initial and terminal subcompartments are the same, say $\ell_{k}$, a simpler notation will be used for the path $p_{\ell_{k}}$. For more than one path in the same subsystem, the path number will be represented by superscript $w ; p_{v_{k} j_{k}}^{w}$. Having the connection and local source be the same, that is, having a path of type $i_{k} \mapsto i_{k}^{*} \rightsquigarrow \cdots$ implies-if not specified differently - that the local input will be taken as the subthroughflow into that subcompartment, $\check{\tau}_{i_{k}}=\tau_{i_{k}}$.

The subsystems can be decomposed into subflows and the associated substorages generated by these subflows along a set of mutually exclusive and exhaustive directed subflow paths. By mutually exclusive subflow paths, we mean that no given subflow path in a subsystem is a subpath, that is, completely inside of another path in the same subsystem. The exhaustiveness, in this context, means that such mutually exclusive subflow paths all together sum to the entire subsystem so partitioned. A subflow path that does not self intersect will be called the linear path, and the one with the same initial and terminal subcompartment will be called the closed path. A subflow path composed of linear and closed subpaths will be called the mixed type path. We will use the notation of $P_{k}$ for a set of mutually exclusive and exhaustive subflow paths in subsystem $k$, and $w_{k}$ for the number of paths in this set.

Each subsystem can be partitioned along a set of mutually exclusive and exhaustive subflow paths as follows: For subsystem $k$, subcompartment $k_{k}$ can be taken as the connection of all subflow paths with the local input being environmental input $z_{k}$. The terminal subcompartments of linear and closed subpaths can be taken as the subcompartments with environmental output and the initial subcompartments, respectively. The cumulative transient outflow at the terminal subcompartment of a closed subpath will be considered as the local output. Consequently, the number of subflow paths in a subsystem obtained by this partitioning is equal to the number of local outputs. This subsystem partitioning will be called the natural subsystem partitioning. The natural subsystem partitioning of all subsystems yields a mutually exclusive and exhaustive partitioning of the entire system.

S2.2. Dynamic subsystem partitioning. The transient subflows and associated substorages along a subflow path between two subcompartments have recently been introduced by $[10,12]$ as summarized below.

Along a given subflow path $p_{n_{k} j_{k}}^{w}=i_{k} \mapsto j_{k} \rightarrow \ell_{k} \rightarrow n_{k}$, the transient inflow at subcompartment $\ell_{k}, f_{\ell_{k} j_{k} i_{k}}^{w}(t)$, generated by the local input from $i_{k}$ to $j_{k}$ during $\left[t_{1}, t\right]$, $t_{1} \geq t_{0}$, is the input segment that is transmitted from $j_{k}$ to $\ell_{k}$ at time $t$. Similarly, the transient outflow generated by the transient inflow at $\ell_{k}$ during $\left[t_{1}, t\right], f_{n_{k} \ell_{k} j_{k}}^{w}(t)$, is the inflow segment that is transmitted from $\ell_{k}$ to the next subcompartment, $n_{k}$, along the path at time $t$. The associated transient substorage in subcompartment $\ell_{k}$ at time $t, x_{n_{k} \ell_{k} j_{k}}^{w}(t)$, is then the substorage segment governed by the transient inflow and outflow balance during $\left[t_{1}, t\right]$.

The transient outflow at subcompartment $\ell_{k}$ at time $t, f_{n_{k} \ell_{k} j_{k}}^{w}(t)$, along subflow path $p_{n_{k} i_{k}}^{w}$ from $j_{k}$ to $n_{k}$ can be formulated as follows:

$$
f_{n_{k} \ell_{k} j_{k}}^{w}(t)=\frac{x_{n_{k} \ell_{k} j_{k}}^{w}(t)}{x_{\ell_{k}}(t)} f_{n_{k} \ell_{k}}(t, \mathrm{x})
$$


where the transient substorage, $x_{n_{k} \ell_{k} j_{k}}^{w}(t)$, is determined by the governing equation

$$
\dot{x}_{n_{k} \ell_{k} j_{k}}^{w}(t)=f_{\ell_{k} j_{k} i_{k}}^{w}(t)-\frac{\hat{\tau}_{\ell_{k}}(t, x)}{x_{\ell_{k}}(t)} x_{n_{k} \ell_{k} j_{k}}^{w}(t), \quad x_{n_{k} \ell_{k} j_{k}}^{w}\left(t_{1}\right)=0 .
$$

The governing equations, Eqs. S2.1 and S2.2, establishes the foundation of the $d y$ namic subsystem partitioning (see Fig. 3). These equations for each subcompartment along a given flow path of interest will then be coupled with the partitioned or original system and be solved simultaneously.

S2.3. Static subsystem partitioning. The static version of this dynamic subsystem partitioning methodology is introduced in Section 2.4. Additional relationships are formulated in this section.

S2.3.1. Transient flows and storages. Since time derivatives are zero at steady state, we set $\dot{x}_{n_{k} \ell_{k} j_{k}}^{w}(t)=0$ in Eq. S2.2. Then, the static transient outflow, $f_{n_{k} \ell_{k} j_{k}}^{w}$, at subcompartment $\ell_{k}$ along subflow path $p_{n_{k} i_{k}}^{w}$ from $j_{k}$ to $n_{k}$, and the transient substorage, $x_{n_{k} \ell_{k} j_{k}}^{w}$, generated in $\ell_{k}$ by the transient inflow, $f_{\ell_{k} j_{k} i_{k}}^{w}$, are formulated as follows:

$$
x_{n_{k} \ell_{k} j_{k}}^{w}=\frac{x_{\ell}}{\tau_{\ell}} f_{\ell_{k} j_{k} i_{k}}^{w} \quad \text { and } \quad f_{n_{k} \ell_{k} j_{k}}^{w}=\frac{f_{n \ell}}{x_{\ell}} x_{n_{k} \ell_{k} j_{k}}^{w}=\frac{f_{n \ell}}{\tau_{\ell}} f_{\ell_{k} j_{k} i_{k}}^{w} .
$$

The second equality for $f_{n_{k} \ell_{k} j_{k}}^{w}$ is obtained by using the first equation for $x_{n_{k} \ell_{k} j_{k}}^{w}$ in the first equality. The relationships in Eq. S2.3 establish the foundation of the static subsystem partitioning.

Self-intersecting directed flow paths are common within compartmental systems. The transient inflows and outflows and associated transient substorages change with each cycle along such paths. The cumulative values are obtained by summing up all transient subflows and substorages at the corresponding subcompartments with each cycle. The number of terms in these summations depends on the number of times the directed path pass through the corresponding subcompartments. In particular, transient subflows cycle along directed closed paths repeatedly and indefinitely. Therefore, in this case, the summations yield a convergent infinite geometric series, due to their construction.

The sum of the transient inflows from subcompartment $j_{k}$ to $\ell_{k}$ and the outflows from $\ell_{k}$ to $n_{k}$ generated at subcompartment $\ell_{k}$ by the local input into the connection of a given non-self-intersecting closed subflow path $p_{n_{k} j_{k}}^{w}$ will respectively be called the inward and outward cumulative transient subflow at subcompartment $\ell_{k}$. The associated storage generated by the inward cumulative transient sublow will be called cumulative transient substorage. These inward and outward cumulative transient subflows will be denoted by $\check{\tau}_{\ell_{k}}^{w}$ and $\hat{\tau}_{\ell_{k}}^{w}$, respectively, and the cumulative transient substorage by $x_{\ell_{k}}^{w}$. They can be formulated as

$$
x_{\ell_{k}}^{w}=\sum_{m=1}^{m_{w}} x_{n_{k} \ell_{k} j_{k}}^{w, m}, \quad \check{\tau}_{\ell_{k}}^{w}=\sum_{m=1}^{m_{w}^{w}} f_{\ell_{k} j_{k} i_{k}}^{w, m}, \quad \text { and } \quad \hat{\tau}_{\ell_{k}}^{w}=\sum_{m=1}^{m_{w}} f_{n_{k} \ell_{k} j_{k}}^{w, m},
$$

for $k=0,1, \ldots, n$, where the superscript $m$ represents the cycle number, and $m_{w}$ is the number of cycles, that is, the number of times the path $p_{n_{k} i_{k}}^{w}$ pass through subcompartment $\ell_{k}$. Large number of terms, $m_{w}$, in computation of these summations reduce truncation errors and, thus, improve the approximations [10, 12]. 
S3. The diact flows and storages.

The static diact flows are defined and derivation for indirect flows and storages are presented in detail in Section 2.6. Parallel derivation for the other static diact flows and storages are formulated in Appendix E. Note that the diact flows and storages are developed for throughflows in the present manuscript. Similar formulations relative to external inputs an outputs can also be developed, as done for the indirect flows by [14]. The dynamic version of this static approach is discussed further below.

In this section, we will introduce an alternative path-based static diact flow and storage formulation, which is originally proposed for dynamic nonlinear systems by $[10,12]$. A hypothetical model is analyzed as an illustrative example for the comparison of both of these static and path-based approaches in Case Study S5.2.

S3.1. Path-based formulations. Based on the subsystem partitioning methodology, five important transaction types for ecological systems are introduced by [10, 12]: the dynamic diact flows and storages. The static versions of the dynamic transfer flows (denoted by $\mathrm{t}$ ) and the associated storages generated by the transfer flows are formulated below. Parallel derivation of path-based static direct (d), indirect (i), cycling (c), and acyclic (a) flows and storages are straightforward.

The composite transfer subflow will be defined as the total intercompartmental transient flow from one subcompartment, directly or indirectly through other subcompartments, to another in the same subsystem. Let $P_{i_{k} j_{k}}^{\mathrm{t}}$ be the set of mutually exclusive subflow paths $p_{i_{k} j_{k}}^{w}$ from subcompartment $j_{k}$ directly or indirectly to $i_{k}$ in subsystem $k$. The composite transfer subflow from subcompartment $j_{k}$ to $i_{k}, \tau_{i_{k} j_{k}}^{\mathrm{t}}$, can be expressed as the sum of the cumulative transient subflows, $\check{\tau}_{i_{k}}^{w}$, generated by the outward subthroughflow at subcompartment $j_{k}, \hat{\tau}_{j_{k}}$, and transmitted into $i_{k}$ along all subflow paths $p_{i_{k} j_{k}}^{w} \in P_{i_{k} j_{k}}^{\mathrm{t}}$. The associated composite transfer substorage, $x_{i_{k} j_{k}}^{\mathrm{t}}$, at subcompartment $i_{k}$ is the sum of the cumulative transient substorages, $x_{i_{k}}^{w}$, generated by the cumulative transient inflows, $\check{\tau}_{i_{k}}^{w}$. Alternatively, $x_{i_{k} j_{k}}^{\mathrm{t}}$ can be defined as the storage segment generated by the composite transfer inflow $\tau_{i_{k} j_{k}}^{\mathrm{t}}$ in subcomaprtment $i_{k}$. The composite transfer subflow and substorage will then be formulated as follows:

$$
\tau_{i_{k} j_{k}}^{\mathrm{t}}=\sum_{w=1}^{w_{k}} \check{\tau}_{i_{k}}^{w} \quad \text { and } \quad x_{i_{k} j_{k}}^{\mathrm{t}}=\sum_{w=1}^{w_{k}} x_{i_{k}}^{w}
$$

where $w_{k}$ is the number of subflow paths $p_{i_{k} j_{k}}^{w} \in P_{i_{k} j_{k}}^{\mathrm{t}}$. The sum of all composite transfer subflows and substorages from subcompartment $j_{k}$ to $i_{k}$ for each subsystem $k$ are called the composite transfer flow and storage from compartment $j$ to $i, \tau_{i j}^{\mathrm{t}}$ and $x_{i j}^{\mathrm{t}}$, and formulated as

$$
\tau_{i j}^{\mathrm{t}}=\sum_{k=0}^{n} \tau_{i_{k} j_{k}}^{\mathrm{t}} \quad \text { and } \quad x_{i j}^{\mathrm{t}}=\sum_{k=0}^{n} x_{i_{k} j_{k}}^{\mathrm{t}} .
$$

For notational convenience we define $n \times n$ matrix measures $T_{k}^{\mathrm{t}}$ and $X_{k}^{\mathrm{t}}$ whose $(i, j)$-elements are $\tau_{i_{k} j_{k}}^{\mathrm{t}}$ and $x_{i_{k} j_{k}}^{\mathrm{t}}$, respectively. That is,

$$
T_{k}^{\mathrm{t}}=\left(\tau_{i_{k} j_{k}}^{\mathrm{t}}\right) \quad \text { and } \quad X_{k}^{\mathrm{t}}=\left(x_{i_{k} j_{k}}^{\mathrm{t}}\right) .
$$

These measures $T_{k}^{\mathrm{t}}$ and $X_{k}^{\mathrm{t}}$ will, accordingly, be called the $k^{\text {th }}$ composite transfer subflow and associated substorage matrices. The corresponding composite transfer flow and associated storage matrix measures are $T^{\mathrm{t}}=\left(\tau_{i j}^{\mathrm{t}}\right)$ and $X^{\mathrm{t}}=\left(x_{i j}^{\mathrm{t}}\right)$, respectively. 
TABLE S1

The dynamic diact flow distribution and the simple and composite diact (sub)flow matrices. The superscript $\left(^{*}\right)$ in each equation represents any of the diact symbols.

\begin{tabular}{cll}
\hline diact & flow distribution matrix & flows \\
\hline $\mathrm{d}$ & $N^{\mathrm{d}}=F \mathcal{T}^{-1}$ & \\
$\mathrm{i}$ & $N^{\mathrm{i}}=\tilde{T} \hat{\mathrm{T}}^{-1}-F \mathcal{T}^{-1}$ & $T^{*}=N^{*}\left(\mathcal{T}-\hat{\mathcal{T}}_{0}\right)$ \\
$\mathrm{a}$ & $N^{\mathrm{a}}=\tilde{T} \hat{\mathrm{T}}^{-1}-\tilde{\mathrm{T}} \hat{\mathrm{T}}^{-1} \hat{T} \hat{\mathrm{T}}^{-1}$ & $T_{\ell}^{*}=N^{*} \hat{\mathcal{T}}_{\ell}$ \\
$\mathrm{c}$ & $N^{\mathrm{c}}=\tilde{\mathrm{T}} \hat{\mathrm{T}}^{-1} \hat{T} \hat{\mathrm{T}}^{-1}$ & $\tilde{T}^{*}=N^{*} \hat{\mathrm{T}}$ \\
$\mathrm{t}$ & $N^{\mathrm{t}}=\tilde{T} \hat{\mathrm{T}}^{-1}$ & \\
\hline
\end{tabular}

Let $P_{i_{k} j_{k}}^{\mathrm{d}}$ and $P_{i_{k} j_{k}}^{\mathrm{i}}$ be defined as the sets of mutually exclusive direct and indirect subflow paths $p_{i_{k} j_{k}}^{w}$ from subcompartment $j_{k}$, directly and indirectly, to $i_{k}$, respectively. The sets $P_{i_{k} j_{k}}^{\mathrm{c}}$ and $P_{i_{k} j_{k}}^{\mathrm{a}}$ are also defined as the sets of mutually exclusive cycling and acyclic subflow paths $p_{i_{k} j_{k}}^{w}$ from $j_{k}$ to $i_{k}$ with a closed and linear subpath at terminal subcompartment $i_{k}$, respectively. The cycling subflow set, $P_{i_{k}}^{\mathrm{c}}$, can alternatively be defined as the set of mutually exclusive subflow paths $p_{i_{k}}^{w}$ from subcompartment $i_{k}$ indirectly back to itself. The number of subflow paths in $P_{i_{k} j_{k}}^{*}$ will be denoted by $w_{k}$, where the superscript $\left({ }^{*}\right)$ represent any of the diact symbols.

The transfer flows, associated storages, and corresponding matrix measures are formulated in Eqs. S3.1, S3.2, and S3.3, using the transfer subflow set, $P_{i_{k} j_{k}}^{\mathrm{t}}$. All the other diact flows, associated storages, and matrix measures can then be formulated similarly by substituting the corresponding diact flows and storages for their transfer counterparts in these equations and by using the corresponding diact subflow sets instead. In path-based formulation, the local inputs for the simple and composite diact subflows are $\hat{\tau}_{k_{k}}$ and $\hat{\tau}_{j_{k}}$, and the corresponding subflow sets are $P_{i_{k} k_{k}}^{*}$ and $P_{i_{k} j_{k}}^{*}$, respectively. Note that, for the cycling case, the first entrance of the transient subflows and substorages into $i_{k}$ are not considered as cycling subflows and substorages.

It is worth emphasizing that the signs of the diact flows and storages graph theoretically indicate the existence of the directed diact paths between the corresponding compartments (nodes or vertices). For example, the relationship $\operatorname{sgn}\left(\tau_{i j}^{\mathbf{i}}\right)>0$ shows that there is at least one indirect path from node $j$ to $i$, where $\operatorname{sgn}(\cdot)$ is the sign function.

S3.2. Dynamic approach. Through an alternative dynamic approach, explicit formulations for the dynamic diact flows have recently been developed by [10]. These dynamic diact flows are listed in Table S1. The dynamic diact storages can be computed as formulated in Eq. S2.2, with the inflows being the corresponding diact flows. If the system is linear, the diact storages can be computed explicitly and analytically as well [10]. The new diagonal matrices used in the table are defined as follows:

$$
\check{\mathbf{T}}(t, \mathrm{x})=\operatorname{diag}(\check{T}(t, \mathrm{x})), \quad \hat{\mathbf{T}}(t, \mathrm{x})=\operatorname{diag}(\hat{T}(t, \mathrm{x})), \quad \tilde{\mathbf{T}}(t, \mathrm{x})=\operatorname{diag}(\tilde{T}(t, \mathrm{x})) .
$$

For the sake of readability, the function arguments are dropped in Table S1.

\section{S4. Output-oriented system analysis.}

The output-oriented system analysis is introduced in Appendix F. In the context of the proposed methodology, the main difference between the formulations of the input- and output-oriented analyses is that, in the latter case, the flow regime is 
conceptually reversed, that is,

$$
\bar{F}=F^{T}, \quad \overline{\mathcal{Z}}=\mathcal{Y}, \quad \text { and } \quad \overline{\mathcal{Y}}=\mathcal{Z} .
$$

To distinguish input- and output-oriented quantities, we use the bar notation over the output-oriented ones.

In parallel to the input-oriented static solutions given in Eq. 2.13 and 2.15, the output-oriented substorage matrix and initial stocks, $\bar{X}$ and $\bar{x}_{0}$, at steady state become

$$
\begin{aligned}
\bar{X} & =-\bar{A}^{-1} \overline{\mathcal{Z}}=\overline{\mathcal{X}}(\overline{\mathcal{T}}-\bar{F})^{-1} \overline{\mathcal{Z}} \\
& =-\bar{A}^{-1} \mathcal{Y}=\mathcal{X}\left(\mathcal{T}-F^{T}\right)^{-1} \mathcal{Y} \text { and } \quad \bar{x}_{0}=\mathbf{0}
\end{aligned}
$$

where

$$
\bar{A}=(\bar{F}-\overline{\mathcal{T}}) \overline{\mathcal{X}}^{-1}=\left(F^{T}-\mathcal{T}\right) \mathcal{X}^{-1}=F^{T} \mathcal{X}^{-1}-\mathcal{R}^{-1} .
$$

The output-oriented substorage and subthroughflow matrices and initial subthroughflow vector can then be written as

$$
\begin{aligned}
& \check{\bar{T}}=\mathcal{Y}+F^{T} \mathcal{X}^{-1} \bar{X}, \\
& \hat{\bar{T}}=(\mathcal{Z}+\operatorname{diag}(F \mathbf{1})) \mathcal{X}^{-1} \bar{X}=\mathcal{T} \mathcal{X}^{-1} \bar{X}=\mathcal{R}^{-1} \bar{X}, \\
& \bar{\tau}_{0}=\check{\bar{\tau}}_{0}=\hat{\bar{\tau}}_{0}=\mathbf{0} .
\end{aligned}
$$

Therefore, the output-oriented substorage and subthroughflow matrices, $\bar{X}$ and $\bar{T}$, obtained by the output-oriented system partitioning methodology can be expressed by the relationships given in Eqs. S4.2 and S4.3, as introduced in Eq. F.6 and F.7.

S4.1. Output-oriented subsystem scaling. The output-oriented cumulative storage and flow distribution matrices, $\bar{S}$ and $\bar{N}$, similar to the definitions of $S$ and $N$ in Eq. 2.18, can be formulated using the subsystem scaling argument as follows:

$$
\begin{aligned}
\bar{S} & =\bar{X} \mathcal{Y}^{-1} \\
\check{\bar{N}} & =\check{\bar{T}} \mathcal{Y}^{-1}=I+F^{T} \mathcal{X}^{-1} \bar{X} \mathcal{Y}^{-1}, \\
\hat{\bar{N}} & =\check{\bar{T}} \mathcal{Y}^{-1}=(\mathcal{Z}+\operatorname{diag}(F \mathbf{1})) \mathcal{X}^{-1} X \mathcal{Y}^{-1},
\end{aligned}
$$

where $\mathcal{Y}$ is invertible. As immediate consequences of these definitions,

$$
\bar{X}=\bar{S} \mathcal{Y} \quad \Rightarrow \quad x=\bar{S} y,
$$

and, since $\bar{T}=\check{\bar{T}}=\hat{\bar{T}}$ implies that $\bar{N}=\check{\bar{N}}=\hat{\bar{N}}$, we have

$$
\bar{T}=\bar{N} \mathcal{Y} \quad \Rightarrow \quad \tau=\bar{N} y .
$$

From Eqs. S4.4, we also have the output-oriented counterpart of Eq. 2.26:

$$
\bar{T}=\mathcal{R}^{-1} \bar{X} \Rightarrow \bar{S}=\mathcal{R} \bar{N} .
$$

Since $\bar{T}=\check{\bar{T}}=\hat{\bar{T}}$ at steady state, using the $\hat{\bar{T}}$ formulation in Eq. S4.3, $\check{\bar{T}}$ can be written as

$$
\bar{T}=\mathcal{Y}+F^{T} \mathcal{T}^{-1} \bar{T} \Rightarrow \bar{T}=\left(I-F^{T} \mathcal{T}^{-1}\right)^{-1} \mathcal{Y}
$$


The cumulative flow distribution matrix $\bar{N}$ defined in Eq. S4.4 can be expressed in the following various forms using the relationships given in Eqs. S4.3 and S4.6:

$$
\bar{N}=\bar{T} \mathcal{Y}^{-1}=I+F^{T} \mathcal{X}^{-1} \bar{X} \mathcal{Y}^{-1}=\mathcal{R}^{-1} \bar{S}=\left(I-\bar{Q}^{\tau}\right)^{-1}
$$

where $\bar{Q}^{\tau}=F^{T} \mathcal{T}^{-1}$. The solution for the substorage matrix given in Eq. S4.2 also imply that the cumulative storage distribution matrix defined in Eq. S4.4 can be expressed as

$$
\bar{S}=\bar{X} \mathcal{Y}^{-1}=-\bar{A}^{-1}=\mathcal{X}\left(\mathcal{T}-F^{T}\right)^{-1}=\mathcal{R}\left(I-\bar{Q}^{\tau}\right)^{-1}=(I-\bar{L})^{-1} \mathcal{R} .
$$

Since the flow intensity matrix, $\bar{A}$, is diagonally dominant, it is invertible. Using the relationship given in Eq. A.5 for linear systems with constant input, $\bar{S}$ can alternatively be expressed at steady state as follows:

$$
\bar{S}=\lim _{t \rightarrow \infty} \int_{t_{0}}^{t} \mathrm{e}^{(t-s) \bar{A}} d s=\bar{\Omega} \lim _{t \rightarrow \infty}\left(\int_{t_{0}}^{t} \mathrm{e}^{(t-s) \bar{\Lambda}} d s\right) \bar{\Omega}^{-1}
$$

where the matrices $\bar{\Omega}$ and $\bar{\Lambda}$ are the eigenvector and eigenvalue matrices of the diagonalizable flow intensity matrix $\bar{A}$.

Note that, the counterparts of the proposed output-oriented cumulative storage and flow distribution matrices, $\bar{S}$ and $\bar{N}$, in the environ theory are denoted by $S^{\prime}$ and $N^{\prime}$, respectively [38]. These matrix measures are related as follows:

$$
N^{\prime}=\bar{N}^{T} \text { and } S^{\prime}=\bar{S}^{T} .
$$

S4.2. Output-oriented subsystem partitioning. The output-oriented subsystem partitioning methodology can be formulated similar to the input-oriented subsystem partitioning introduced in Section 2.4. The output-oriented subsystem partitioning enables backtracking the fate of arbitrary storages and the associated inflows generating these storages at each compartment along a given flow path.

Along a given path $\bar{p}_{n_{k} j_{k}}^{w}=i_{k} \mapsto j_{k} \rightarrow \ell_{k} \rightarrow n_{k}$, the transient outflow $\bar{f}_{n_{k} \ell_{k} j_{k}}^{w}$ at subcompartment $\ell_{k}$ along subflow path $\bar{p}_{n_{k} j_{k}}^{w}$ from $j_{k}$ to $n_{k}$ and the transient substorage $\bar{x}_{n_{k} \ell_{k} j_{k}}^{w}$ generated at $\ell_{k}$ by the transient inflow $\bar{f}_{\ell_{k} j_{k} i_{k}}^{w}$ can be formulated as follows:

$$
\bar{x}_{n_{k} \ell_{k} j_{k}}^{w}=\frac{x_{\ell}}{\tau_{\ell}} \bar{f}_{\ell_{k} j_{k} i_{k}}^{w} \quad \text { and } \quad \bar{f}_{n_{k} \ell_{k} j_{k}}^{w}=\frac{f_{n \ell}}{x_{\ell}} \bar{x}_{n_{k} \ell_{k} j_{k}}^{w}=\frac{f_{n \ell}}{\tau_{\ell}} \bar{f}_{\ell_{k} j_{k} i_{k}}^{w} .
$$

The storage and outward throughflow matrices generated by inward throughflow matrix at step $(m)$ along all subflow paths of the output-oriented natural subsystem partitioning can then be formulated in matrix form as

$$
\bar{X}^{(m)}=\mathcal{R} \bar{T}^{(m)} \quad \text { and } \quad \bar{T}^{(m+1)}=\bar{Q}^{\tau} \bar{T}^{(m)}=\bar{Q}^{x} \bar{X}^{(m)}
$$

where the output-oriented flow and storage distribution matrices are $\bar{Q}^{x}=F^{T} \mathcal{X}^{-1}$, $\bar{Q}^{\tau}=F^{T} \mathcal{T}^{-1}=\bar{Q}^{x} \mathcal{R}$. These relationships imply that $\bar{X}^{(m+1)}=\bar{L} \bar{X}^{(m)}$ where $\bar{L}=\mathcal{R} \bar{Q}^{x}$. If the distribution matrices are invertible, backtracking from the $(m+1)^{s t}$ to the $m^{t h}$ step is possible using Eq. S4.11.

Similar to the infinite series expansions of the input-oriented substorage and subthroughflow matrices formulated in Eqs. D.2 and D.3, the output-oriented substorage and subthroughflow matrices can also be expressed as infinite geometric series:

$$
\bar{T}=\bar{N} \mathcal{Y}=\left(I-\bar{Q}^{\tau}\right)^{-1} \mathcal{Y}=\left(\sum_{m=0}^{\infty}\left(\bar{Q}^{\tau}\right)^{m}\right) \mathcal{Y}
$$


TABLE S2

The output-oriented, storage-based diact flow and storage distribution and the simple and composite diact (sub)flow and (sub)storage matrices. The superscript $\left(^{*}\right)$ in each equation represents any of the diact symbols.

\begin{tabular}{crrrr}
\hline diact & flow and storage distribution matrices & flows & storages \\
\hline $\mathrm{d}$ & $\bar{S}^{\mathrm{d}, x}=F^{T} \mathcal{X}^{-1}$ & & \\
$\mathrm{i}$ & $\bar{S}^{\mathrm{i}, x}=\left(\mathcal{R}^{-1} \bar{S}-I\right) \mathcal{S}^{-1}-F^{T} \mathcal{X}^{-1}$ & & $\bar{T}^{*}=\bar{S}^{*, x} \mathcal{X}$ & $\bar{X}^{*}=\bar{N}^{*, x} \mathcal{X}$ \\
$\mathrm{a}$ & $\bar{S}^{\mathrm{a}, x}=\left(\mathcal{S}^{-1} \bar{S}-I\right) \mathcal{S}^{-1}$ & $\bar{N}^{*, x}=\mathcal{R} \bar{S}^{*, x}$ & $\bar{T}_{\ell}^{*}=\bar{S}^{*, x} \overline{\mathcal{X}}_{\ell}$ & $\bar{X}_{\ell}^{*}=\bar{N}^{*, x} \overline{\mathcal{X}}_{\ell}$ \\
$\mathrm{c}$ & $\bar{S}^{\mathrm{c}, x}=\left(\mathcal{R}^{-1} \bar{S}-\mathcal{S}^{-1} \bar{S}\right) \mathcal{S}^{-1}$ & & $\tilde{\bar{T}}^{*}=\bar{S}^{*, x} \bar{X}$ & $\tilde{\bar{X}}^{*}=\bar{N}^{*, x} \bar{X}$ \\
$\mathrm{t}$ & $\bar{S}^{\mathrm{t}, x}=\left(\mathcal{R}^{-1} \bar{S}-I\right) \mathcal{S}^{-1}$ & & \\
\hline
\end{tabular}

and

$$
\bar{X}=\bar{S} \mathcal{Y}=\mathcal{R}\left(I-\bar{Q}^{\tau}\right)^{-1} \mathcal{Y}=(I-\bar{L})^{-1} \mathcal{R} \mathcal{Y}=\left(\sum_{m=0}^{\infty} \bar{L}^{m}\right) \mathcal{R} \mathcal{Y}
$$

using Eq. S4.11.

S4.3. Output-oriented diact flows and storages. The derivation of the output-oriented diact transaction types is straightforward, in parallel to their inputoriented counterparts. We will only derive the output-oriented simple cycling flows and storages below as an example.

The input-oriented cycling subflow and substorage are defined as reflexive indirect or transfer subflow from a subcompartment to itself and associated storage generated by this subflow. The output-oriented cycling subflow and associated substorage can be formulated in line with this definition. The output-oriented cycling subflow from an output-producing subcompartment $i_{i}$ to itself can be expressed as follows:

$$
\bar{\tau}_{i_{i}}^{\mathrm{c}}=\bar{\tau}_{i_{i} i_{i}}^{\mathrm{t}}=\bar{\tau}_{i_{i} i_{i}}^{\mathrm{i}}=\sum_{j=1}^{n} \bar{f}_{i_{i} j_{i}}=\sum_{j=1}^{n} f_{j_{i} i_{i}}=\bar{\tau}_{i_{i}}-y_{i}
$$

The composite subflow parallel to $\bar{\tau}_{i_{i}}^{\mathrm{c}}$ in subsystem $k, \bar{\tau}_{i_{k}}^{\mathrm{c}}$, and the cycling flow at compartment $i, \bar{\tau}_{i}^{\mathrm{c}}=\bar{\tau}_{i i}^{\mathrm{c}}$, are related as

$$
\bar{\tau}_{i}^{\mathrm{c}}=\sum_{k=1}^{n} \bar{\tau}_{i_{k}}^{\mathrm{c}}=\sum_{k=1}^{n}\left(\bar{\tau}_{i_{i}}-y_{i}\right) \frac{\bar{\tau}_{i_{k}}}{\bar{\tau}_{i_{i}}}=\frac{\bar{\tau}_{i_{i}}-y_{i}}{\bar{\tau}_{i_{i}}} \sum_{k=1}^{n} \bar{\tau}_{i_{k}}=\frac{\bar{\tau}_{i_{i}}-y_{i}}{\bar{\tau}_{i_{i}}} \bar{\tau}_{i}=\bar{n}_{i}^{\mathrm{c}} \tau_{i}
$$

where $\bar{n}_{i}^{\mathrm{c}}=\left(\bar{\tau}_{i_{i}}-y_{i}\right) / \bar{\tau}_{i_{i}}$ will be called the output-oriented cycling flow distribution factor. Note that, the relationship $z_{i} / \tau_{i_{i}}=y_{i} / \bar{\tau}_{i_{i}}$ given in Eq. G.7 results in the equality of $n_{i}^{c}=\bar{n}_{i}^{c}$. The proportionality formualted in Eq. E.3 also implies that the cycling storage at compartment $i, \bar{x}_{i}^{\mathrm{c}}=\bar{x}_{i i}^{\mathrm{c}}$, becomes

$$
\bar{x}_{i_{k}}^{\mathrm{c}}=\bar{\tau}_{i_{k}}^{\mathrm{c}} \frac{\bar{x}_{i}}{\bar{\tau}_{i}}=\bar{\tau}_{i_{k}}^{\mathrm{c}} \frac{x_{i}}{\tau_{i}} \quad \text { and } \quad \bar{x}_{i}^{\mathrm{c}}=\sum_{k=1}^{n} \bar{x}_{i_{k}}^{\mathrm{c}}=\bar{\tau}_{i}^{\mathrm{c}} \frac{\bar{x}_{i}}{\bar{\tau}_{i}}=\bar{\tau}_{i}^{\mathrm{c}} \frac{x_{i}}{\tau_{i}} .
$$

The equality $n_{i}^{\mathrm{c}}=\bar{n}_{i}^{\mathrm{c}}$ derived above can be expressed in matrix form as $\mathcal{N}=\overline{\mathcal{N}}$, which is also consistent with Eq. G.4. This relationship can be used to demonstrate the equivalence of the input and output-oriented cycling flow and storage distribution matrices, that is, $\mathcal{N}^{c}=\overline{\mathcal{N}}^{c}=(\mathcal{N}-I) \mathcal{N}^{-1}$ and $\mathcal{S}^{c}=\overline{\mathcal{S}}^{\mathrm{c}}=\mathcal{R} \mathcal{N}^{\mathrm{c}}$. The equivalence of the input- and output-oriented cycling distribution matrices, however, does not 
imply the equivalence of the corresponding subflow and substorage matrices. This is because of the fact that $T \neq \bar{T}$, as discussed in Eq. F.4. That is,

$$
\tilde{T}^{\mathrm{c}}=\mathcal{N}^{\mathrm{c}} T \neq \mathcal{N}^{\mathrm{c}} \bar{T}=\tilde{\bar{T}}^{\mathrm{c}} \quad \text { and } \quad \tilde{X}^{\mathrm{c}}=\mathcal{S}^{\mathrm{c}} T \neq \mathcal{S}^{\mathrm{c}} \bar{T}=\tilde{\bar{X}}^{\mathrm{c}} .
$$

Similarly, we have $\tilde{T}^{\mathrm{a}} \neq \tilde{\bar{T}}^{\mathrm{a}}$ and $\tilde{X}^{\mathrm{a}} \neq \tilde{\bar{X}}^{\mathrm{a}}$. On the other hand, since $\tau=\bar{\tau}$,

$$
\tilde{\tau}^{\mathrm{c}}=\tilde{\bar{\tau}}^{\mathrm{c}}, \quad \tilde{x}^{\mathrm{c}}=\tilde{\bar{x}}^{\mathrm{c}}, \quad \tilde{\tau}^{\mathrm{a}}+z=\tilde{\bar{\tau}}^{\mathrm{a}}+y, \quad \text { and } \quad \tilde{x}^{\mathrm{a}}+\mathcal{R} z=\tilde{\bar{x}}^{\mathrm{a}}+\mathcal{R} y .
$$

These relationships indicate that, the simple cycling throughflows are the same in either orientation, and the simple acyclic throughflows are the same up to the corresponding environmental input. In other words, the amount of environmental inputs that transit through the system compartments are the same in both input- and output-orientations, as well as the remaining portion of the inputs cycling within the system.

One of the implications of this observation is that the input-oriented transient subflows at the terminal compartment and the output-oriented transient subflows at the initial compartment along a linear flow path are the same. Due to a similar reasoning, along all possible flow paths, it can also be shown that

$$
T^{\mathrm{d}}=\left(\bar{T}^{\mathrm{d}}\right)^{T} \quad \text { and } \quad T^{\mathrm{a}}=\left(\bar{T}^{\mathrm{a}}\right)^{T} .
$$

These relationships, however, are not true for the corresponding composite substorages, that is $X^{\mathrm{d}} \neq\left(\bar{X}^{\mathrm{d}}\right)^{T}$ and $X^{\mathrm{a}} \neq\left(\bar{X}^{\mathrm{a}}\right)^{T}$, because of the differences in the compartmental residence times.

The output-oriented, flow-based diact flow and storage distribution matrices, as well as the corresponding simple and composite flows and storages are listed in Table 3 at both compartmental and subcompartmental levels. Similar to the input-oriented, storage-based diact flows and storages introduced in Section E.6, the output-oriented storage-based diact transactions can also be formulated. These matrix measures are listed in Table S2.

\section{S5. Case studies.}

Two case studies are presented in this section. The first case study presents the analytic solution to a linear ecosystem model with time dependent input. The second case study presents various aspects of the proposed methodology that are not covered in the other case studies. In particular, the path-based and static approaches in computation of the static diact flows and storages are compared in this example.

S5.1. Case study. In this case study, a linear ecosystem model introduced by [24] is solved analytically. This dynamic model has two compartments, $x_{1}(t)$ and $x_{2}(t)$ (see Fig. S1). The flow regime is described as

$$
F(t)=\left[\begin{array}{cc}
0 & \frac{2}{3} x_{2}(t) \\
\frac{4}{3} x_{1}(t) & 0
\end{array}\right], \quad z(t)=\left[\begin{array}{c}
z_{1}(t) \\
z_{2}(t)
\end{array}\right], \quad y(t)=\left[\begin{array}{c}
\frac{1}{3} x_{1}(t) \\
\frac{5}{3} x_{2}(t)
\end{array}\right] .
$$

The governing equations take the following form:

$$
\begin{aligned}
& \dot{x}_{1}(t)=z_{1}(t)+\frac{2}{3} x_{2}(t)-\left(\frac{4}{3}+\frac{1}{3}\right) x_{1}(t) \\
& \dot{x}_{2}(t)=z_{2}(t)+\frac{4}{3} x_{1}(t)-\left(\frac{2}{3}+\frac{5}{3}\right) x_{2}(t)
\end{aligned}
$$




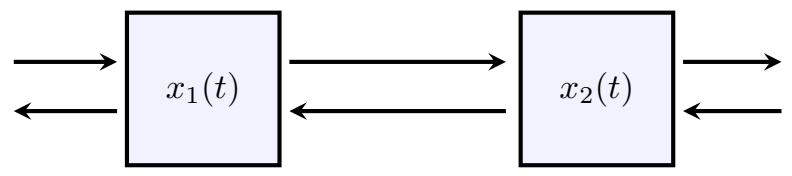

FIG. S1. Schematic representation of the model network (Case Study S5.1).

with the initial conditions $\left[x_{1,0}, x_{2,0}\right]^{T}=[3,3]^{T}$.

The subcompartmentalization yields the substates that represent the substorage values as follows:

$$
x_{1_{k}}(t) \quad \text { and } \quad x_{2_{k}}(t), \quad k=0,1,2 .
$$

The flow partitioning can then be formulated as

$$
F_{k}(t)=\left[\begin{array}{cc}
0 & \frac{2}{3} d_{2_{k}} x_{2} \\
\frac{4}{3} d_{1_{k}} x_{1} & 0
\end{array}\right], \quad \check{z}_{k}(t)=\left[\begin{array}{c}
\delta_{1 k} z_{1} \\
\delta_{2 k} z_{2}
\end{array}\right], \quad \hat{y}_{k}(t)=\left[\begin{array}{c}
\frac{1}{3} d_{1_{k}} x_{1} \\
\frac{5}{3} d_{2_{k}} x_{2}
\end{array}\right] .
$$

Consequently, the dynamic system partitioning methodology yields the following governing system of equations:

$$
\begin{aligned}
& \dot{x}_{1_{k}}(t)=z_{1_{k}}(t)+\frac{2}{3} x_{2_{k}}(t)-\left(\frac{4}{3}+\frac{1}{3}\right) x_{1_{k}}(t) \\
& \dot{x}_{2_{k}}(t)=z_{2_{k}}(t)+\frac{4}{3} x_{1_{k}}(t)-\left(\frac{2}{3}+\frac{5}{3}\right) x_{2_{k}}(t)
\end{aligned}
$$

with the initial conditions

$$
x_{i_{k}}\left(t_{0}\right)= \begin{cases}3, & k=0 \\ 0, & k \neq 0\end{cases}
$$

for $i=1,2$. There are $n \times(n+1)=2 \times 3=6$ equations in the system.

The system can be written in matrix form as formulated in Eq. A.1:

$$
\begin{aligned}
\dot{X}(t) & =\mathcal{Z}(t)+A X(t), & X\left(t_{0}\right) & =\mathbf{0} \\
\dot{x}_{0}(t) & =A x_{0}(t), & & x_{0}\left(t_{0}\right)=x_{0}
\end{aligned}
$$

where the constant flow intensity matrix $A$, as defined in Eq. 2.12, becomes

$$
A=\left[\begin{array}{cc}
-(4 / 3+1 / 3) & 2 / 3 \\
4 / 3 & -(2 / 3+5 / 3)
\end{array}\right]=\left[\begin{array}{cc}
-5 / 3 & 2 / 3 \\
4 / 3 & -7 / 3
\end{array}\right] .
$$

The partitioned governing system, Eq. S5.2, is linear. We can, therefore, solve the system analytically as explained in Appendix A. Since the flow intensity matrix $A$ is constant, we have the following fundamental matrix solution as formulated in Eq. A.3:

$$
V(t)=\left[\begin{array}{cc}
\frac{2 \mathrm{e}^{-t}}{3}+\frac{\mathrm{e}^{-3 t}}{3} & \frac{\mathrm{e}^{-t}}{3}-\frac{\mathrm{e}^{-3 t}}{3} \\
\frac{2 \mathrm{e}^{-t}}{3}-\frac{2 \mathrm{e}^{-3 t}}{3} & \frac{\mathrm{e}^{-t}}{3}+\frac{2 \mathrm{e}^{-3 t}}{3}
\end{array}\right] .
$$

For $z=[1,1]^{T}$, the solutions for the matrix equation, Eq. S5.2, then become

$$
X(t)=\left[\begin{array}{cc}
\frac{7}{9}-\frac{\mathrm{e}^{-3 t}}{9}-\frac{2 \mathrm{e}^{-t}}{3} & \frac{2}{9}+\frac{\mathrm{e}^{-3 t}}{9}-\frac{\mathrm{e}^{-t}}{3} \\
\frac{4}{9}+\frac{2 \mathrm{e}^{-3 t}}{9}-\frac{2 \mathrm{e}^{-t}}{3} & \frac{5}{9}-\frac{2 \mathrm{e}^{-3 t}}{9}-\frac{\mathrm{e}^{-t}}{3}
\end{array}\right], \quad x_{0}(t)=\left[\begin{array}{c}
3 \mathrm{e}^{-t} \\
3 \mathrm{e}^{-t}
\end{array}\right],
$$


as given in Eq. A.4. Therefore, the solution to the original system, Eq. S5.1, is

$$
x(t)=x_{0}(t)+X(t) \mathbf{1}=\left[\begin{array}{l}
x_{1}(t) \\
x_{2}(t)
\end{array}\right]=\left[\begin{array}{l}
2 \mathrm{e}^{-t}+1 \\
2 \mathrm{e}^{-t}+1
\end{array}\right] .
$$

The subthroughflow matrices, $\check{T}(t, \mathrm{x})$ and $\hat{T}(t, \mathrm{x})$, can be expressed as formulated in Eq. 2.11 using the solution for the substorage matrix $X(t)$.

The steady-state solutions of the system, as formulated in Eq. 2.13, then become

$$
X=-A^{-1}=\mathcal{X}(\mathcal{T}-F)^{-1}=\left[\begin{array}{ll}
7 / 9 & 2 / 9 \\
4 / 9 & 5 / 9
\end{array}\right] \text { and } \quad x_{0}=\mathbf{0} .
$$

It can easily be seen that, this steady-state solution is the same as the limit of the dynamic solution, Eq. S5.3, as $t$ tends to infinity. That is, $\lim _{t \rightarrow \infty} X(t)=X$.

We also analyzed the system with a time dependent input $z(t)=[3+\sin (t), 3+$ $\sin (2 t)]^{T}$, as suggested by [24]. Similar computations with the same fundamental matrix, $V(t)$, lead us to the following initial substorage vector and substorage matrix components:

$$
\begin{aligned}
& x_{1_{0}}(t)=x_{2_{0}}(t)=3 \mathrm{e}^{-t}, \\
& x_{1_{1}}(t)=\frac{7}{3}-\frac{11 \cos (t)}{30}+\frac{13 \sin (t)}{30}-\frac{5 \mathrm{e}^{-t}}{3}-\frac{3 \mathrm{e}^{-3 t}}{10}, \\
& x_{1_{2}}(t)=\frac{2}{3}-\frac{16 \cos (2 t)}{195}-\frac{2 \sin (2 t)}{195}-\frac{13 \mathrm{e}^{-t}}{15}+\frac{11 \mathrm{e}^{-3 t}}{39}, \\
& x_{2_{1}}(t)=\frac{4}{3}-\frac{4 \cos (t)}{15}+\frac{2 \sin (t)}{15}-\frac{5 \mathrm{e}^{-t}}{3}+\frac{3 \mathrm{e}^{-3 t}}{5}, \\
& x_{2_{2}}(t)=\frac{5}{3}-\frac{46 \cos (2 t)}{195}+\frac{43 \sin (2 t)}{195}-\frac{13 \mathrm{e}^{-t}}{15}-\frac{22 \mathrm{e}^{-3 t}}{39} .
\end{aligned}
$$

From these solutions, we can express the solutions to the original system, Eq. S5.1, as follows:

$$
x_{1}(t)=\sum_{k=0}^{2} x_{1_{k}}(t) \quad \text { and } \quad x_{2}(t)=\sum_{k=0}^{2} x_{2_{k}}(t) .
$$

The proposed dynamic method solves linear systems analytically. Analytical solutions can be used to compute the quantities in question at any time $t$.

The elements of the inward subthroughflow vector, $\check{\tau}_{0}(t)$, and subthroughflow matrix, $\check{T}(t)$, can be computed using Eq. 2.11 as follows:

$$
\begin{aligned}
& \check{\tau}_{1_{0}}(t)=2 \mathrm{e}^{-t}, \quad \check{\tau}_{2_{0}}(t)=4 \mathrm{e}^{-t} \\
& \check{\tau}_{1_{1}}(t)=\frac{35}{9}-\frac{8 \cos (t)}{45}+\frac{49 \sin (t)}{45}-\frac{10 \mathrm{e}^{-t}}{9}+\frac{2 \mathrm{e}^{-3 t}}{5}, \\
& \check{\tau}_{1_{2}}(t)=\frac{742}{585}-\frac{184 \cos ^{2}(t)}{585}+\frac{86 \sin (2 t)}{585}-\frac{26 \mathrm{e}^{-t}}{45}-\frac{44 \mathrm{e}^{-3 t}}{117} \\
& \check{\tau}_{2_{1}}(t)=\frac{28}{9}-\frac{22 \cos (t)}{45}+\frac{26 \sin (t)}{45}-\frac{20 \mathrm{e}^{-t}}{9}-\frac{2 \mathrm{e}^{-3 t}}{5} \\
& \check{\tau}_{2_{2}}(t)=\frac{2339}{585}-\frac{128 \cos ^{2}(t)}{585}+\frac{577 \sin (2 t)}{585}-\frac{52 \mathrm{e}^{-t}}{45}+\frac{44 \mathrm{e}^{-3 t}}{117} .
\end{aligned}
$$

With the matrix functions $X(t)$ and $\check{T}(t)$ presented in Eqs. S5.5 and S5.6, the distribution of the environmental inputs and the organization of the associated storages 

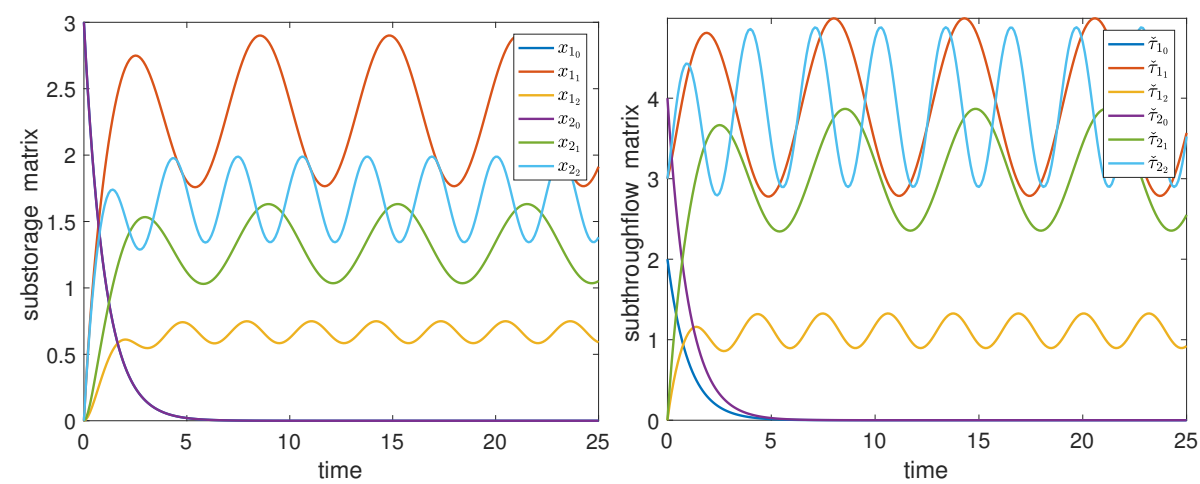

FIG. S2. The graphical representation of the substorage and inward subthroughflow matrices, $X(t)$ and $\check{T}(t)$, for time dependent input $z(t)=[3+\sin (t), 3+\sin (2 t)]^{T}$ (Case Study S5.1).

generated by these inputs within the system are determined individually and separately. Therefore, the evolution of the environmental inputs and associated storages can be tracked individually and separately throughout the system.

The graphical representation of the substorage and inward subthroughflow matrices, $X(t)$ and $\check{T}(t)$, for time dependent input $z(t)=[3+\sin (t), 3+\sin (2 t)]^{T}$, are depicted in Fig. S2.

S5.2. Case study. In this case study, a hypothetical model is analyzed to illustrate various aspects and uses of the proposed methodology that are not discussed in the previous case studies in the present paper. In particular, the correspondence and equivalence of the proposed path-based and static approaches in the formulation of the static diact transactions are demonstrated. Application of the subsystem partitioning methodology will follow the system partitioning.

Let the flow regime of a hypothetical ecosystem model be given as

$$
F=\left[\begin{array}{llll}
0 & 0 & 0 & 0 \\
2 & 0 & 1 & 0 \\
0 & 0 & 0 & 1 \\
0 & 3 & 0 & 0
\end{array}\right], \quad x=\left[\begin{array}{l}
1 \\
2 \\
3 \\
4
\end{array}\right], \quad y=\left[\begin{array}{l}
1 \\
0 \\
0 \\
4
\end{array}\right], \quad z=\left[\begin{array}{l}
3 \\
0 \\
0 \\
2
\end{array}\right]
$$

(see Fig. S3). The subsystem flow information for each subsystem can be computed using Eq. B.1 as follows.

$$
\begin{gathered}
F_{1}=\left[\begin{array}{cccc}
0 & 0 & 0 & 0 \\
2 & 0 & 1 / 2 & 0 \\
0 & 0 & 0 & 1 / 2 \\
0 & 5 / 2 & 0 & 0
\end{array}\right], \quad F_{4}=\left[\begin{array}{cccc}
0 & 0 & 0 & 0 \\
0 & 0 & 1 / 2 & 0 \\
0 & 0 & 0 & 1 / 2 \\
0 & 1 / 2 & 0 & 0
\end{array}\right], \quad F_{2}=F_{3}=\mathbf{0} . \\
\hat{y}_{1}=\left[\begin{array}{l}
1 \\
0 \\
0 \\
2
\end{array}\right], \quad \hat{y}_{4}=\left[\begin{array}{l}
0 \\
0 \\
0 \\
2
\end{array}\right], \quad \check{z}_{1}=\left[\begin{array}{l}
1 \\
0 \\
0 \\
2
\end{array}\right], \quad \check{z}_{4}=\left[\begin{array}{l}
0 \\
0 \\
0 \\
2
\end{array}\right],
\end{gathered}
$$

and $\hat{y}_{2}=\hat{y}_{3}=\check{z}_{2}=\check{z}_{3}=\mathbf{0}$. The flows and storages in the initial subsystem $(k=0)$ vanish at steady state, that is $x_{0}=\tau_{0}=\mathbf{0}$. 


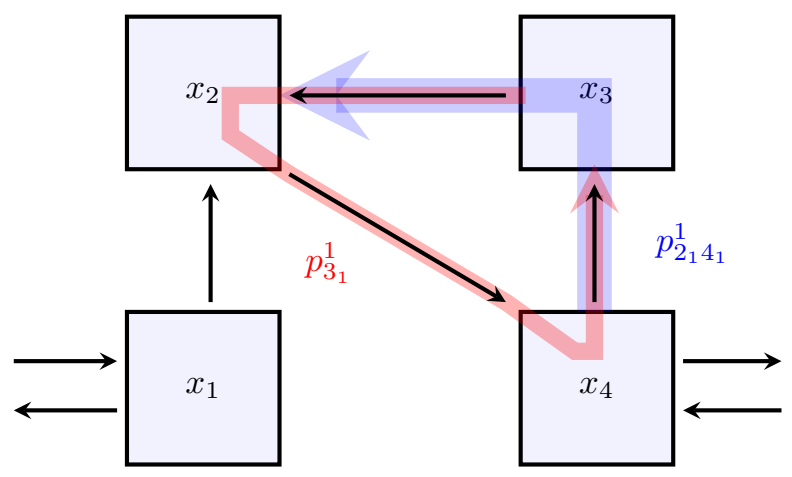

FIG. S3. Schematic representation of the model network. Along subflow paths $p_{2_{1} 4_{1}}^{1}$ (blue) and $p_{3_{1}}^{1}$ (red) the respective indirect and cycling flows and associated substorages are computed (subsystems are not shown) (Case Study S5.2).

The steady-state solution to the governing system Eq. 2.6 with the initial conditions $x_{0}=[1,2,3,4]^{T}$, as given in Eq. 2.13, becomes

$$
X=-A^{-1} \mathcal{Z}=\left[\begin{array}{cccc}
1 & 0 & 0 & 0 \\
5 / 3 & 0 & 0 & 1 / 3 \\
3 / 2 & 0 & 0 & 3 / 2 \\
2 & 0 & 0 & 2
\end{array}\right] \quad \text { where } \quad A=\left[\begin{array}{cccc}
-3 & 0 & 0 & 0 \\
2 & -3 / 2 & 1 / 3 & 0 \\
0 & 0 & -1 / 3 & 1 / 4 \\
0 & 3 / 2 & 0 & -5 / 4
\end{array}\right]
$$

and $\mathcal{Z}=\operatorname{diag}(z)$. From Eq. 2.15, the subthroughflow matrix, $T$, and throughflow vector, $\tau$, can be expressed as

$$
T=\mathcal{Z}+F \mathcal{X}^{-1} X=\left[\begin{array}{cccc}
3 & 0 & 0 & 0 \\
5 / 2 & 0 & 0 & 1 / 2 \\
1 / 2 & 0 & 0 & 1 / 2 \\
5 / 2 & 0 & 0 & 5 / 2
\end{array}\right] \text { and } \tau=T \mathbf{1}=\left[\begin{array}{c}
3 \\
3 \\
1 \\
5
\end{array}\right]
$$

The storage and flow distribution matrices formulated in Eqs. 2.23 and 2.22, $S=$ $-A^{-1}$ and $N=\left(I-F \mathcal{T}^{-1}\right)^{-1}$, become

$$
S=\left[\begin{array}{cccc}
1 / 3 & 0 & 0 & 0 \\
5 / 9 & 5 / 6 & 5 / 6 & 1 / 6 \\
1 / 2 & 3 / 4 & 15 / 4 & 3 / 4 \\
2 / 3 & 1 & 1 & 1
\end{array}\right] \text { and } N=\left[\begin{array}{cccc}
1 & 0 & 0 & 0 \\
5 / 6 & 5 / 4 & 5 / 4 & 1 / 4 \\
1 / 6 & 1 / 4 & 5 / 4 & 1 / 4 \\
5 / 6 & 5 / 4 & 5 / 4 & 5 / 4
\end{array}\right]
$$

Application of the subsystem partitioning methodology will also be presented below for this system. The transient outflow from $2_{1}$ to $4_{1}$ through a short directed subflow path $p_{4_{1} 1_{1}}^{1}=1_{1} \mapsto 1_{1} \rightsquigarrow 2_{1} \rightarrow 4_{1}$ of length 2 and associated substorage can be computed as given in Eq. S2.3:

$$
x_{4_{1} 2_{1} 1_{1}}^{1}=\frac{x_{2}}{\tau_{2}} f_{2_{1} 1_{1} 1_{1}}^{1}=\frac{4}{3} \quad \text { and } \quad f_{4_{1} 2_{1} 1_{1}}^{1}=\frac{f_{42}}{\tau_{2}} f_{2_{1} 1_{1} 1_{1}}^{1}=2 .
$$

Note that the local input for this subflow path is $\tau_{1_{1}}$ and $f_{2_{1} 1_{1} 1_{1}}^{1}=f_{2_{1} 1_{1}}^{1}=2$ (see Section S2.1). Using Eq. 2.31, the same computation can be done as follows:

$$
x_{4_{1} 2_{1} 1_{1}}^{1}=r_{2} q_{21}^{\tau} \tau_{1_{1}}=\frac{4}{3} \quad \text { and } \quad f_{4_{1} 2_{1} 1_{1}}^{1}=q_{42}^{\tau} q_{21}^{\tau} \tau_{1_{1}}=2
$$


where the residence time and flow distribution matrices are $\mathcal{R}=\operatorname{diag}([1 / 3,2 / 3,3,4 / 5])$ and

$$
Q^{\tau}=\left[\begin{array}{cccc}
0 & 0 & 0 & 0 \\
2 / 3 & 0 & 1 & 0 \\
0 & 0 & 0 & 1 / 5 \\
0 & 1 & 0 & 0
\end{array}\right]
$$

Along another directed path $p_{2_{1} 1_{1}}^{1}=0_{1} \mapsto 1_{1} \rightarrow 2_{1} \rightsquigarrow 4_{1} \rightsquigarrow 3_{1} \rightarrow 2_{1}$ in subsystem 1 with local input $z_{1}$, we will also compute the inward and outward cumulative transient subflows, $\check{\tau}_{2_{1}}^{1}$ and $\hat{\tau}_{2_{1}}^{1}$, and associated substorage at subcompartment $2_{1}, x_{2_{1}}^{1}$, using Eqs. S2.3 and S2.4. Note that $p_{2_{1} 1_{1}}^{1}$ is a flow path of mixed type, that is, it has a closed subpath. The links contributing to the transient substorage, $x_{2_{1}}^{1}$, on this closed subpath are marked with red cycle numbers in the extended path diagram below:

$$
p_{2_{1} 1_{1}}^{1}=0_{1} \mapsto 1_{1} \rightarrow 2_{1} \rightsquigarrow 4_{1} \rightsquigarrow 3_{1} \stackrel{1}{\rightarrow} 2_{1} \rightsquigarrow 4_{1} \rightsquigarrow 3_{1} \stackrel{2}{\rightarrow} 2_{1} \rightsquigarrow 4_{1} \rightsquigarrow 3_{1} \stackrel{3}{\rightarrow} 2_{1} \rightarrow \cdots
$$

Therefore, using the sum of a geometric series formula, $\sum_{m=0}^{\infty} a r^{m}=\frac{a}{1-r},|r|<1$, we have

$$
\begin{aligned}
x_{2_{1}}^{1}=x_{4_{1} 2_{1} 1_{1}}^{1}+\sum_{m=1}^{\infty} x_{4_{1} 2_{1} 3_{1}}^{1, m} & =\frac{4}{3}+\frac{4}{3}\left(\frac{1}{5}+\frac{1}{5^{2}}+\cdots+\frac{1}{5^{m}}+\cdots\right) \\
& =\frac{4}{3} \cdot \frac{5}{4}=\frac{5}{3}=x_{2_{1}}, \\
\check{\tau}_{2_{1}}^{1}=f_{2_{1} 1_{1} 0_{1}}^{1}+\sum_{m=1}^{\infty} f_{2_{1} 3_{1} 4_{1}}^{1, m} & =2+2\left(\frac{1}{5}+\frac{1}{5^{2}}+\cdots+\frac{1}{5^{m}}+\cdots\right) \\
& =2 \cdot \frac{5}{4}=\frac{5}{2}=\tau_{2_{1}}, \\
\hat{\tau}_{2_{1}}^{1}=f_{4_{1} 2_{1} 1_{1}}^{1}+\sum_{m=1}^{\infty} f_{4_{1} 2_{1} 3_{1}}^{1, m} & =2+2\left(\frac{1}{5}+\frac{1}{5^{2}}+\cdots+\frac{1}{5^{m}}+\cdots\right) \\
& =2 \cdot \frac{5}{4}=\frac{5}{2}=\tau_{2_{1}} .
\end{aligned}
$$

In the summation above, the infinite upper limit $\left(m_{w}=\infty\right)$ is due to the closed subpath. These results indicate that the transient subflows along the given subflow path generate inward and outward cumulative transient subflows at subcompartment $2_{1}$ that are equal to each other and also to the subthroughflow at that subcompartment. That is, $\tilde{\tau}_{2_{1}}^{1}=\tau_{2_{1}}=\hat{\tau}_{2_{1}}^{1}$. This is the case for not only the infinite sum, but also with each cycle $m$, because of the steady-state condition and the fact that $p_{2_{1} 1_{1}}^{1}$ is a subflow path that completely represents the subflow regime in subsystem 1 . The substorage generated by the inward cumulative transient subflow, $x_{2_{1}}^{1}$, is equal to the substorage value, $x_{2_{1}}$, as expected, for the same reason. That is, $x_{2_{1}}^{1}=x_{2_{1}}$. These equalities indicate that the system and subsystem partitioning methodologies yield the same results and are integrated within the unifying framework of the proposed mathematical method.

Alternatively, using the distribution factors and Eq. 2.31 instead, the same quan- 
tities can be computed as follows:

$$
\begin{aligned}
& x_{2_{1}}^{1}=r_{2} \check{\tau}_{2_{1}}^{1}=\frac{2}{3} \cdot \frac{5}{2}=\frac{5}{3}=x_{2_{1}} \text { where } \\
& \check{\tau}_{2_{1}}^{1}=z_{1} q_{21}^{\tau} \frac{1}{1-q_{42}^{\tau} q_{34}^{\tau} q_{23}^{\tau}}=3 \cdot \frac{2}{3} \cdot \frac{5}{4}=\frac{5}{2}=\tau_{2_{1}}, \\
& \hat{\tau}_{2_{1}}^{1}=z_{1} q_{21}^{\tau} q_{42}^{\tau} \frac{1}{1-q_{42}^{\tau} q_{34}^{\tau} q_{23}^{\tau}}=3 \cdot \frac{2}{3} \cdot 1 \cdot \frac{5}{4}=\frac{5}{2}=\tau_{2_{1}} .
\end{aligned}
$$

The indirect flow and storage from compartment 4 to 2 will also be computed using both path-based and static approaches below for a comparison (see Fig. S3). The indirect flows $\tau_{2_{1} 4_{1}}^{\mathrm{i}}$ and $\tau_{2_{4} 4_{4}}^{\mathrm{i}}$ along the corresponding subflow paths $p_{2_{1} 4_{1}}^{2}=4_{1} \mapsto$ $4_{1} \rightsquigarrow 3_{1} \rightarrow 2_{1}$ and $p_{2_{4} 4_{4}}^{2}=4_{4} \mapsto 4_{4} \rightsquigarrow 3_{4} \rightarrow 2_{4}$ can be computed using the distribution factors. The corresponding local inputs are $\tau_{4_{1}}$ and $\tau_{4_{4}}$, respectively. Therefore, we have

$$
\tau_{2_{1} 4_{1}}^{\mathrm{i}}=\tau_{4_{1}} q_{34}^{\tau} q_{23}^{\tau}=\frac{1}{2}=\tau_{4_{4}} q_{34}^{\tau} q_{23}^{\tau}=\tau_{2_{4} 4_{4}}^{\mathrm{i}}, \quad x_{2_{1}}^{\mathrm{i}}=r_{2} \tau_{2_{1} 4_{1}}^{\mathrm{i}}=\frac{1}{3}=r_{2} \tau_{2_{4} 4_{4}}^{\mathrm{i}}=x_{2_{4}}^{\mathrm{i}} .
$$

A geometric series formulation as in Eq. S5.12 is not suitable here because compartment 4 is on the closed paths, and $\tau_{4_{1}}$ and $\tau_{4_{4}}$ include all cycling flows along the paths in the corresponding subsystems.

The indirect flow and storage $\tau_{24}^{\mathrm{i}}$ and $x_{24}^{\mathrm{i}}$ then become

$$
\tau_{24}^{\mathrm{i}}=\check{\tau}_{2_{1} 4_{1}}^{1}+\check{\tau}_{2_{4} 4_{4}}^{1}=\frac{1}{2}+\frac{1}{2}=1=f_{23} \quad \text { and } \quad x_{24}^{\mathrm{i}}=x_{2_{1}}^{1}+x_{2_{4}}^{1}=\frac{1}{3}+\frac{1}{3}=\frac{2}{3} .
$$

using Eqs. S3.2. Note that $\tau_{24}^{\mathrm{i}}=f_{23}$ as expected, since compartment 3 has no input, and $f_{23}$ is the only indirect flow transmitted from compartment 4 to 2 . The same results can be obtained at the compartmental level along flow path $p_{24}^{2}=4 \mapsto 4 \rightsquigarrow$ $3 \rightarrow 2$ with local input $\tau_{4}$ instead:

$$
\tau_{24}^{\mathrm{i}}=\tau_{4} q_{34}^{\tau} q_{23}^{\tau}=1 \quad \text { and } \quad x_{2}^{\mathrm{i}}=r_{2} \tau_{24}^{\mathrm{i}}=\frac{2}{3} .
$$

The same indirect flow and storage computations can be done more practically using the proposed static approach formulated in Eqs. 2.38 and 2.41. That is,

$$
\tau_{24}^{\mathrm{i}}=n_{24}^{\mathrm{i}} \tau_{4}=\frac{\tau_{2_{4}}-f_{2_{4} 4_{4}}}{\tau_{4_{4}}} \tau_{4}=\frac{1}{5} \cdot 5=1=f_{23} \quad \text { and } \quad x_{24}^{\mathrm{i}}=\tau_{24}^{\mathrm{i}} \frac{x_{2}}{\tau_{2}}=1 \cdot \frac{2}{3}=\frac{2}{3} .
$$

Clearly, the results are the same in both proposed path-based and static approaches, which justifies both formulations. Using the diact flow and storage formulations listed in Table 1, the indirect flows and storages for the entire system can also be computed as follows:

$$
T^{\mathrm{i}}=\left[\begin{array}{cccc}
0 & 0 & 0 & 0 \\
1 / 2 & 3 / 5 & 0 & 1 \\
1 / 2 & 3 / 5 & 1 / 5 & 0 \\
5 / 2 & 0 & 1 & 1
\end{array}\right] \quad \text { and } \quad X^{\mathrm{i}}=\left[\begin{array}{cccc}
0 & 0 & 0 & 0 \\
1 / 3 & 2 / 5 & 0 & 2 / 3 \\
3 / 2 & 9 / 5 & 3 / 5 & 0 \\
2 & 0 & 4 / 5 & 4 / 5
\end{array}\right] .
$$

The cycling subflows at subcompartments $3_{1}$ and $3_{4}$ will be computed, similar to the indirect flow computations above. The set of subflow paths that contribute to the simple cycling subflows at subcompartments $3_{1}$ and $3_{4}$ are $P_{3_{1} 1_{1}}=\left\{p_{3_{1} 1_{1}}^{1}\right\}$ and 
$P_{3_{4} 4_{4}}=\left\{p_{3_{4} 4_{4}}^{1}\right\}$ where $p_{3_{1} 1_{1}}^{1}=0_{1} \mapsto 1_{1} \rightsquigarrow 2_{1} \rightsquigarrow 4_{1} \rightarrow 3_{1} \rightsquigarrow 2_{1} \rightsquigarrow 4_{1} \rightarrow 3_{1}$ and $p_{3_{4} 4_{4}}^{1}=0_{4} \mapsto 4_{4} \rightarrow 3_{4} \rightsquigarrow 2_{4} \rightsquigarrow 4_{4} \rightarrow 3_{4}$ (see Fig. S3). The links contributing to the simple cycling subflows, $\tau_{3_{1}}^{c}$ and $\tau_{3_{4}}^{c}$, on subflow paths $p_{3_{1} 1_{1}}^{1}$ and $p_{3_{4} 4_{4}}^{1}$ are marked with red cycle numbers in the extended diagram below:

$$
\begin{aligned}
& p_{3_{1} 1_{1}}^{1}=0_{1} \mapsto 1_{1} \rightsquigarrow 2_{1} \rightsquigarrow 4_{1} \stackrel{0}{\rightarrow} 3_{1} \rightsquigarrow 2_{1} \rightsquigarrow 4_{1} \stackrel{1}{\rightarrow} 3_{1} \rightsquigarrow 2_{1} \rightsquigarrow 4_{1} \stackrel{2}{\rightarrow} 3_{1} \rightsquigarrow 2_{1} \rightsquigarrow \cdots \\
& p_{3_{4} 4_{4}}^{1}=0_{4} \mapsto 4_{4} \stackrel{0}{\rightarrow} 3_{4} \rightsquigarrow 2_{4} \rightsquigarrow 4_{4} \stackrel{1}{\rightarrow} 3_{4} \rightsquigarrow 2_{4} \rightsquigarrow 4_{4} \stackrel{2}{\rightarrow} 3_{4} \rightsquigarrow 2_{4} \rightsquigarrow 4_{4} \stackrel{3}{\rightarrow} 3_{4} \rightsquigarrow \cdots
\end{aligned}
$$

Note that the initial inflows are not counted as cycling flow and, therefore, the corresponding noncontributing links are indexed by 0. As formulated in Eqs. S3.1,

$$
x_{3_{k}}^{\mathrm{c}}=\sum_{w=1}^{1} x_{3_{k}}^{w}=x_{3_{k}}^{1} \quad \text { and } \quad \tau_{3_{k}}^{\mathrm{c}}=\sum_{w=1}^{1} \check{\tau}_{3_{k}}^{w}=\check{\tau}_{3_{k}}^{1}
$$

for $k=1,4$ where $w_{k}=1$ is the number of subflow paths in $P_{3_{1} 1_{1}}$ and $P_{3_{4} 4_{4}}$.

Along subflow path $p_{31_{1}}^{1}$, using Eqs. S2.3 and S2.4, the cumulative transient storage in and inward throughflow at subcompartment $3_{1}$ become

$$
\begin{aligned}
& x_{3_{1}}^{1}=\sum_{m=1}^{\infty} x_{2_{1} 3_{1} 4_{1}}^{1, m}=\frac{6}{25}\left(1+\frac{1}{5}+\frac{1}{5^{2}}+\cdots+\frac{1}{5^{m}}+\cdots\right)=\frac{6}{25} \cdot \frac{5}{4}=\frac{3}{10} \\
& \check{\tau}_{3_{1}}^{1}=\sum_{m=1}^{\infty} f_{3_{1} 4_{1} 2_{1}}^{1, m}=\frac{2}{25}\left(1+\frac{1}{5}+\frac{1}{5^{2}}+\cdots+\frac{1}{5^{m}}+\cdots\right)=\frac{2}{25} \cdot \frac{5}{4}=\frac{1}{10}
\end{aligned}
$$

where the infinite upper limit, $m_{1}=\infty$, is due to the closed subpath. Similarly, along $p_{34_{4}}^{1}$, we have

$$
x_{34}^{1}=\sum_{m=1}^{\infty} x_{2_{4} 3_{4} 4_{4}}^{1, m}=\frac{6}{25} \cdot \frac{5}{4}=\frac{3}{10} \quad \text { and } \quad \check{\tau}_{34}^{1}=\sum_{m=1}^{\infty} f_{3_{4} 4_{4} 2_{4}}^{1, m}=\frac{2}{25} \cdot \frac{5}{4}=\frac{1}{10} .
$$

Consequently, the simple cycling storage in and flow at compartment 3 becomes

$$
\begin{aligned}
& x_{3}^{c}=\sum_{k=1}^{n} x_{3_{k}}^{w}=x_{3_{1}}^{1}+x_{3_{4}}^{1}=\frac{3}{10}+\frac{3}{10}=\frac{3}{5}, \\
& \tau_{3}^{\mathrm{c}}=\sum_{k=1}^{n} \check{\tau}_{3_{k}}^{w}=\check{\tau}_{3_{1}}^{1}+\check{\tau}_{3_{4}}^{1}=\frac{1}{10}+\frac{1}{10}=\frac{1}{5} .
\end{aligned}
$$

Alternatively, the same computation can be done using the distribution factors as formulated in Eq. 2.31 at the compartmental level along closed flow path $p_{3}^{1}=3 \mapsto$ $3 \rightsquigarrow 2 \rightsquigarrow 4 \rightarrow 3$. The simple cycling flow and storage at compartment 3 with local input $\tau_{3}$ become

$$
\tau_{3}^{\mathrm{c}}=\tau_{3} q_{23}^{\tau} q_{42}^{\tau} q_{34}^{\tau}=1 \cdot 1 \cdot 1 \cdot \frac{1}{5}=\frac{1}{5} \quad \text { and } \quad x_{3}^{\mathrm{c}}=r_{3} \tau_{3}^{\mathrm{c}}=3 \cdot \frac{1}{5}=\frac{3}{5} .
$$

The simple cycling flow at and associated storage in compartment 3 can also be computed using the static approach formulated in Eq. E.35:

$$
\tau_{3}^{\mathrm{c}}=n_{3}^{\mathrm{c}} \tau_{3}=\frac{\tau_{3_{3}}-z_{3}}{\tau_{3_{3}}} \tau_{3}=\frac{1}{5} \cdot 1=\frac{1}{5} \quad \text { and } \quad x_{3}^{\mathrm{c}}=r_{3} \tau_{3}^{\mathrm{c}}=\frac{3}{5} .
$$


Note that the results of both proposed path-based and static approaches are the same. Using the diact flow and storage formulations listed in Table 1, the simple cycling flows and storages for the entire system can be computed as follows:

$$
\tilde{T}^{c}=\left[\begin{array}{cccc}
0 & 0 & 0 & 0 \\
1 / 2 & 0 & 0 & 1 / 10 \\
1 / 10 & 0 & 0 & 1 / 10 \\
1 / 2 & 0 & 0 & 1 / 2
\end{array}\right] \text { and } \quad \tilde{X}^{c}=\left[\begin{array}{cccc}
0 & 0 & 0 & 0 \\
1 / 3 & 0 & 0 & 1 / 15 \\
3 / 10 & 0 & 0 & 3 / 10 \\
2 / 5 & 0 & 0 & 2 / 5
\end{array}\right]
$$

The composite cycling flow and storage matrices become

$$
T^{\mathrm{c}}=\left[\begin{array}{cccc}
0 & 0 & 0 & 0 \\
1 / 2 & 3 / 5 & 1 / 5 & 1 / 5 \\
1 / 10 & 3 / 25 & 1 / 5 & 1 / 5 \\
1 / 2 & 3 / 5 & 1 / 5 & 1
\end{array}\right] \text { and } \quad X^{\mathrm{c}}=\left[\begin{array}{cccc}
0 & 0 & 0 & 0 \\
1 / 3 & 2 / 5 & 2 / 15 & 2 / 15 \\
3 / 10 & 9 / 25 & 3 / 5 & 3 / 5 \\
2 / 5 & 12 / 25 & 4 / 25 & 4 / 5
\end{array}\right]
$$

Note that, the simple and composite cycling flows at and storages in compartment 3 are related as $\tau_{33}^{\mathrm{c}}=\tau_{3}^{\mathrm{c}}=\frac{1}{5}$ and $x_{33}^{\mathrm{c}}=x_{3}^{\mathrm{c}}=\frac{3}{5}$.

It is worth emphasizing that the geometric series for the subflow paths in Eqs. S5.11 and S5.13 are the scalar form of the infinite matrix series formulated in Eqs. D.2 and D.3. The common ratio $(1 / 5)$ of the geometric progression is equal to the corresponding cycling flow distribution factors. Since indirect flow $\tau_{24}^{\mathrm{i}}$ is also calculated along the same closed flow path $p_{3}^{1}=3 \mapsto 3 \rightsquigarrow 2 \rightsquigarrow 4 \rightarrow 3$ above, we have the following relationships:

$$
n_{2}^{\mathrm{c}}=n_{3}^{\mathrm{c}}=n_{4}^{\mathrm{c}}=q_{23}^{\tau} q_{42}^{\tau} q_{34}^{\tau}=n_{24}^{\mathrm{i}}=\frac{1}{5} .
$$

Note also that the proposed path-based approach is similar to the average path length computation introduced by [19] which led to the derivation of FCI.

Lastly, we will also present the transfer and acyclic flows in matrix form as formulated in Table 1 below:

$$
T^{\mathrm{t}}=\left[\begin{array}{cccc}
0 & 0 & 0 & 0 \\
5 / 2 & 3 / 5 & 1 & 1 \\
1 / 2 & 3 / 5 & 1 / 5 & 1 \\
5 / 2 & 3 & 1 & 1
\end{array}\right] \text { and } T^{\mathrm{a}}=\left[\begin{array}{cccc}
0 & 0 & 0 & 0 \\
2 & 0 & 4 / 5 & 4 / 5 \\
2 / 5 & 12 / 25 & 0 & 4 / 5 \\
2 & 12 / 5 & 4 / 5 & 0
\end{array}\right]
$$

The $(i, k)$-elements of $T^{\mathrm{t}}$ and $T^{\mathrm{a}}, \tau_{i k}^{\mathrm{t}}$ and $\tau_{i k}^{\mathrm{a}}$, represent the transfer (total) and acyclic flows transmitted from compartment $k$ to $i$. The composite acyclic flow is defined as the transfer flow segment that does not cycle at the terminal compartment $i$. To verify this definition using the path-based approach, the composite cycling flow at compartment 2 transmitted from compartment $3, \tau_{23}^{c}$, can be calculated and used for the computation of acyclic flow in the same direction, $\tau_{23}^{\mathrm{a}}$, as follows:

$$
\tau_{23}^{\mathrm{a}}=\tau_{23}^{\mathrm{t}}-\tau_{23}^{\mathrm{c}}=\frac{4}{5} \quad \text { where } \quad \tau_{23}^{\mathrm{c}}=\tau_{3} q_{23}^{\tau} q_{42}^{\tau} q_{34}^{\tau} q_{23}^{\tau}=\frac{1}{5} .
$$

The static approach is applicable to systems at steady state and more practical to compute, but the path-based approach is applicable to both dynamic and static cases. All the other input- and output-oriented diact flows and storages can be calculated similar to the ones presented above. 


\section{REFERENCES}

[1] T. Allen and M. Giampietro, Holons, creaons, genons, environs, in hierarchy theory: Where we have gone, Ecological Modelling, 293 (2014), pp. 31-41, https://doi.org/10.1016/j. ecolmodel.2014.06.017.

[2] M. Augustinovics, Methods of international and intertemporal comparison of structure, in Contributions to input-output analysis, C. A.P. and B. A., eds., vol. Vol. 1, Amsterdam, 1970, North-Holland Publishing Company, pp. 249-269.

[3] R. Bailey, J. K. Allen, and B. Bras, Applying ecological input-output flow analysis to material flows in industrial systems: Part $i$ : Tracing flows, Journal of Industrial Ecology, 8 (2004), pp. 45-68, https://doi.org/10.1162/1088198041269346.

[4] A. Belgrano, U. M. Scharler, J. Dunne, and R. E. Ulanowicz, Aquatic Food Webs: An Ecosystem Approach, Oxford University Press, Oxford, U.K, 2005.

[5] S. R. Borrett, M. A. Freeze, And A. K. Salas, Equivalence of the realized input and output oriented indirect effects metrics in Ecological Network Analysis, Ecological Modelling, 222 (2011), pp. 2142-2148, https://doi.org/10.1016/j.ecolmodel.2011.04.003, https: //arxiv.org/abs/1103.6276.

[6] S. R. Borrett and M. K. Lau, enaR : An $R$ package for ecosystem network analysis, Methods in Ecology and Evolution, 5 (2014), pp. 1206-1213, https://doi.org/10.1111/2041-210x. 12282.

[7] B. C. Patten and S. J. Whipple, Ecological Utility Analysis: Determination of Interaction Types Between Organisms in Ecosystems, vol. 2, WIT Press, 09 2011, https://doi.org/10. 2495/ECO-V2-N2-88-96.

[8] V. Christensen And D. Pauly, ECOPATH II-a software for balancing steady-state ecosystem models and calculating network characteristics, Ecological modelling, 61 (1992), pp. 169185.

[9] R. R. Christian and C. R. Thomas, Neuse river estuary modeling and monitoring project stage 1: Network analysis for evaluating the consequences of nitrogen loading, Tech. Report UNC-WRRI-2000-325-F, Biology Department, East Carolina University, 2000.

[10] H. Coskun, Dynamic ecological system analysis, Heliyon, 5 (2019), https://doi.org/10.1016/j. heliyon.2019.e02347, https://doi.org/10.31219/osf.io/35xkb.

[11] H. Coskun, Dynamic ecological system measures, Results in Applied Mathematics, 4 (2019), p. 100007, https://doi.org/10.1016/j.rinam.2019.100007, https://doi.org/10.31219/osf.io/ j2pd3.

[12] H. Coskun, Nonlinear decomposition principle and fundamental matrix solutions for dynamic compartmental systems, Discrete and Continuous Dynamical Systems - B, 24 (2019), pp. 6553-6605, https://doi.org/10.3934/dcdsb.2019155, https://doi.org/10.31219/osf.io/ cyrzf.

[13] H. Coskun, Static ecological system measures, Theoretical Ecology, (2019), https://doi.org/ 10.1007/s12080-019-0422-7, https://doi.org/10.31219/osf.io/g4xzt.

[14] H. B. Coskun, Indirect transactions and requirements, OSF Preprints, (2019), https://doi. org/10.31219/osf.io/w2a4d.

[15] B. D. Fath, Network mutualism: Positive community-level relations in ecosystems, Ecological Modelling, 208 (2007), pp. 56-67, https://doi.org/10.1016/j.ecolmodel.2007.04.021.

[16] B. D. Fath And S. R. Borrett, A Matlab® function for Network Environ Analysis, Environmental Modelling and Software, 21 (2006), pp. 375-405, https://doi.org/10.1016/j.envsoft. 2004.11.007.

[17] B. D. Fath And B. C. Patten, Network synergism: Emergence of positive relations in ecological systems, Ecological Modelling, 107 (1998), pp. 127-143, https://doi.org/10.1016/ S0304-3800(97)00213-5.

[18] B. D. Fath And B. C. Patten, Review of Network the Foundations of Environ Analysis, Ecosystems, 2 (1999), pp. 167-179.

[19] J. Finn, Measures of structure and functioning derived from analysis of flows, Journal of Theoretical Biology, 56 (1976), pp. 363-380.

[20] J. T. Finn, Flow Analysis of Models of the Hubbard Brook Ecosystem, Ecology, 61 (1980), pp. 562-571.

[21] B. Hannon, The structure of ecosystems, Journal of theoretical biology, 41 (1973), pp. 535-546.

[22] M. Higashi and B. C. Patten, Dominance of indirect causality in ecosystems, The American Naturalist, 133 (1989), pp. 288-302.

[23] M. Higashi, B. C. Patten, And T. P. Burns, Network trophic dynamics: the modes of energy utilization in ecosystems, Ecological Modelling, 66 (1993), pp. 1-42, https://doi.org/10. 1016/0304-3800(93)90037-S. 
[24] P. W. Hippe, Environ analysis of linear compartmental systems: The dynamic, time-invariant case, Ecological Modelling, 19 (1983), pp. 1-26, https://doi.org/10.1016/0304-3800(83) 90067-4.

[25] H. Hirata AND R. E. Ulanowicz, Information theoretical analysis of the aggregation and hierarchical structure of ecological networks, Journal of Theoretical Biology, 116 (1985), pp. 321-341.

[26] R. Holt, Community modules, in Multitrophic Interactions in Terrestrial Ecosystems, A. Gange and V. Brown, eds., 36th Symposium, British Ecological Society, Blackwell Science, 1997, pp. 333-349.

[27] R. D. Holt, Predation, apparent competition, and the structure of prey communities, Theoretical Population Biology, 12 (1977), pp. 197-229, https://doi.org/10.1016/0040-5809(77) 90042-9, https://arxiv.org/abs/0040580977900429.

[28] C. KazANCI, Network calculations II: a user's manual for EcoNet, in Handbook of Ecological Modelling and Informatics, WITPRESS LTD., 2009, pp. 325-350, https://doi.org/10.2495/ 978-1-84564-207-5/18.

[29] W. W. LEONTIEF, Quantitative input and output relations in the economic systems of the united states, The review of economic statistics, 18 (1936), pp. 105-125.

[30] W. W. LeOnTiEf, Input-output economics, Oxford University Press on Demand, New York, 1986.

[31] R. L. Lindeman, The trophic-dynamic aspect of ecology, Ecology, 23 (1942), pp. 399-417.

[32] Q. Ma AND C. KAZANCI, Analysis of indirect effects within ecosystem models using pathwaybased methodology, Ecological Modelling, 252 (2013), pp. 238-245, https://doi.org/10. 1016/j.ecolmodel.2012.05.002.

[33] J. Matis And B. Patten, Environ analysis of linear compartmental systems: the static, time invariant case, Statistical Ecology, 48 (1981), pp. 527-565.

[34] A. B. Menge, Detection of direct versus indirect effects: were experiments long enough?, AMERICAN NATURALIST, 149 (1997), pp. 801-823.

[35] B. A. Menge, Indirect effects in marine rocky intertidal interaction webs: Patterns and importance, Ecological Monographs, 65 (1995), pp. 21-74, https://doi.org/10.2307/2937158, https://doi.org/10.2307/2937158.

[36] R. T. PAINe, Food web complexity and species diversity, American Naturalist, 100 (1966), pp. 65-75.

[37] B. PAtTen, Energy cycling, length of food chains, and direct versus indirect effects in ecosystems, Canadian Bulletin of Fisheries and Aquatic Sciences, 213 (1985), pp. 119-138.

[38] B. C. PAtten, Systems approach to the concept of environment, Ohio Journal of Science, 78 (1978), pp. 206-222.

[39] B. C. Patten, Energy cycling in the ecosystem, Ecological Modelling, 28 (1985), pp. 1-71, https://doi.org/10.1016/0304-3800(85)90013-4.

[40] B. C. Patten, Energy, emergy and environs, Ecological Modelling, 62 (1992), pp. 29-69, https://doi.org/10.1016/0304-3800(92)90081-O.

[41] B. C. Patten, R. W. Bosserman, J. T. Finn, and W. G. Cale, Propagation of cause in ecosystems, Systems analysis and simulation in ecology, 4 (1976), pp. 457-579.

[42] M. Rasmussen, A. Hastings, M. J. Smith, F. B. Agusto, B. M. Chen-Charpentier, F. M. Hoffman, J. Jiang, K. E. O. Todd-Brown, Y. Wang, Y.-P. Wang, and Y. Luo, Transit times and mean ages for nonautonomous and autonomous compartmental systems, Journal of Mathematical Biology, 73 (2016), pp. 1379-1398, https://doi.org/10.1007/ s00285-016-0990-8, https://doi.org/10.1007/s00285-016-0990-8.

[43] J. R. Schramski, C. Kazanci, And E. W. Tollner, Network environ theory, simulation, and EcoNetß, 2.0, Environmental Modelling and Software, 26 (2011), pp. 419-428, https: //doi.org/10.1016/j.envsoft.2010.10.003.

[44] S. Y. Strauss, Indirect effects in community ecology: Their definition, study and importance., Trends in ecology \& evolution (Personal edition), 6 (1991), pp. 206-210, https://doi.org/ 10.1016/0169-5347(91)90023-Q.

[45] J. Szyrmer and R. E. Ulanowicz, Total flows in ecosystems, Ecological Modelling, 35 (1987), pp. 123-136.

[46] L. J. Tilly, The structure and dynamics of Cone Spring, Ecological Monographs, 38 (1968), pp. 169-197.

[47] L. K. Tuominen, S. J. Whipple, B. C. Patten, Z. Y. Karatas, and C. Kazanci, Contribution of throughflows to the ecological interpretation of integral network utility, Ecological Modelling, 293 (2014), pp. 187-201, https://doi.org/10.1016/j.ecolmodel.2014.01.027.

[48] R. Ulanowicz and C. Puccia, Mixed trophic impacts in ecosystems., Coenoses, 5 (1990), pp. $7-16$. 
[49] R. E. Ulanowicz, Mass and energy flow in closed ecosystems, Journal of Theoretical Biology, 34 (1972), pp. 239-253, https://doi.org/10.1016/0022-5193(72)90158-0.

[50] R. E. Ulanowicz, Quantitative methods for ecological network analysis, Computational Biology and Chemistry, 28 (2004), pp. 321-339, https://doi.org/10.1016/j.compbiolchem.2004. 09.001.

[51] R. E. Ulanowicz, R. D. Holt, and M. BARfiEld, Limits on ecosystem trophic complexity: insights from ecological network analysis, Ecology Letters, 17 (2013), pp. 127-136, https: //doi.org/10.1111/ele.12216.

[52] R. E. Ulanowicz AND J. J. KaY, A package for the analysis of ecosystem flow networks, Environmental Software, 6 (1991), pp. 131-142, https://doi.org/10.1016/0266-9838(91) 90024-K.

[53] J. Wootton, Indirect effects in complex ecosystems: recent progress and future challenges, Journal of Sea Research, 48 (2002), pp. 157-172, https://doi.org/10.1016/S1385-1101(02) 00149-1.

[54] J. T. Wооттоn, Indirect effects and habitat use in an intertidal community: Interaction chains and interaction modifications, The American Naturalist, 141 (1993), pp. 71-89, https://doi.org/10.1086/285461, https://doi.org/10.1086/285461.

[55] J. T. Wootton, The nature and consequences of indirect effects in ecological communities, Annual Review of Ecology and Systematics, 25 (1994), pp. 443-466, https://doi.org/10. 1146/annurev.es.25.110194.002303, https://doi.org/10.1146/annurev.es.25.110194.002303. 